\title{
A Microscopic Picture of Surface Charge Trapping in Semiconductor Nanocrystals
}

\author{
Jonathan Mooney \\ Department of Chemistry \\ McGill University, Montréal
}

February 2014

A thesis submitted to McGill University in partial fulfillment of the requirements of the degree of Doctor of Philosophy

(C) Jonathan Mooney 2014 


\section{Abstract}

The current model for understanding trapping of charge carriers to the surface of semiconductor nanocrystals is inconsistent with experimental evidence indicating that carriers can thermally de-trap from surface sites. A proper understanding of the microscopic details of charge trapping would guide chemical design of the nanocrystal surface for applications such as charge transport, sensing, or photochemistry. This thesis presents a model of surface charge trapping in which transitions to surface state are governed by rates derived from semiclassical electron-transfer theory. In this picture, trapping to the surface induces a strong polarization in the nanocrystal, resulting in a trapped state with strong electron-phonon coupling via the Frölich mechanism. This trapped state then emits over a broad energy range due to a Franck-Condon vibronic progression. This model is shown to be consistent with the temperature-dependence of core and surface emission as well as the spectral properties of surface emission. The strong coupling of the surface state is validated by independent experiments, and the model is shown to hold promise for explaining the experimental data regarding the trapping of hot (excess energy) carriers. 


\section{Résumé}

Le modèle prévalent concernant le piégeage des porteurs de charges à la surface de nanocrystaux semi-conducteurs est inconsistant avec certains résultats expérimentaux indiquant que les charges peuvent subir une relaxation thermique depuis les états de surfaces. Une bonne compréhension des aspects microscopiques du processus de piégeage des porteurs de charges permettrait de guider le design de la surface des nanocrystaux en vue d'applications comme le transport de charges, la détection ou la photo-chimie.

Ce travail de doctorat propose un modèle du piégeage des charges où les taux de transition vers des états de surface sont basés sur la théorie semi-classique du transfert d'électrons. Dans ce modèle, le piégeage à la surface crée une forte polarisation dans le nanocrystal, ce qui résulte en un état piégé avec un grand couplage électron-phonon via le mécanisme de Fröhlich. Cet état piégé émet dans une large bande spectrale due à la progression vibronique de Franck-Condon. Le modèle proposé est consistent avec la dépendance en température du spectre d'émission du centre et de la surface ainsi que des propriétés spectrales d'émission de la surface. Le couplage fort de l'état de surface est validé par des expériences indépendantes et il est montré que le modèle est prometteur pour l'analyse d'expériences sur le piégeage des porteurs de charges chauds (ayant un excès d'énergie). 


\section{Table of Contents}

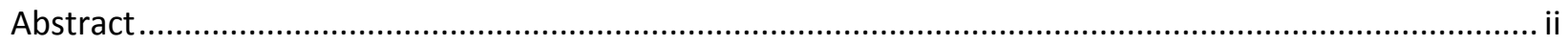

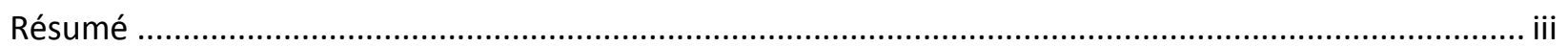

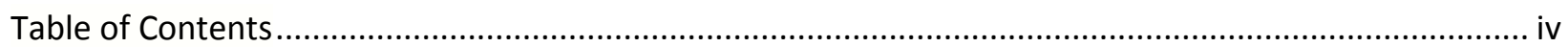

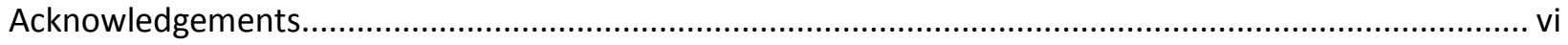

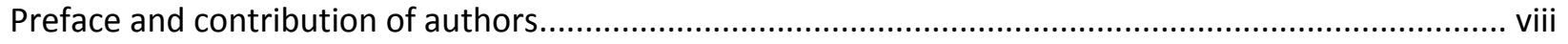

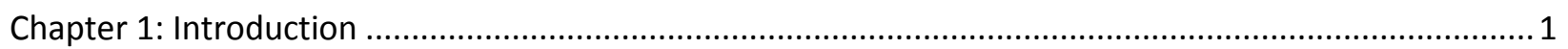

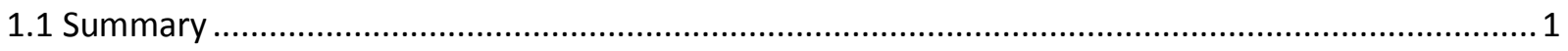

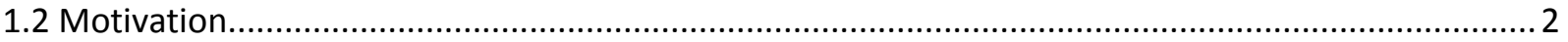

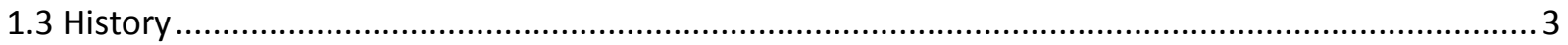

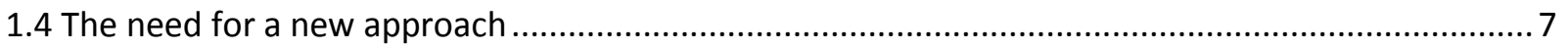

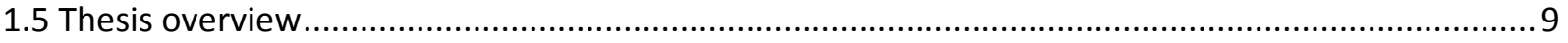

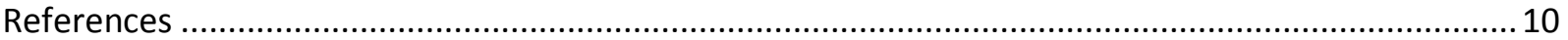

Chapter 2: Electronic structure of semiconductor nanocrystals.......................................................... 13

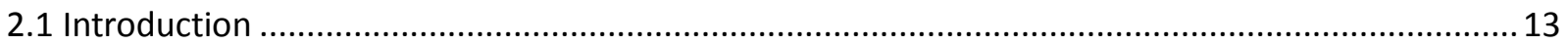

2.2 Semiconductors from bulk to nanoscale.................................................................................. 13

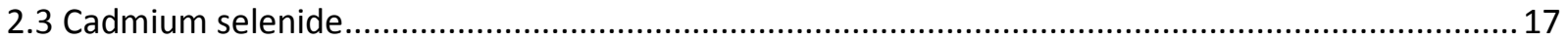

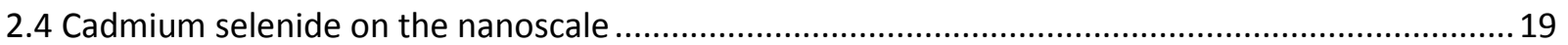

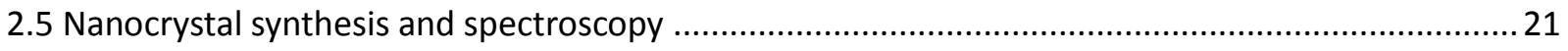

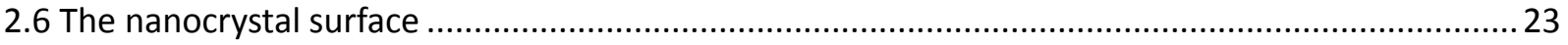

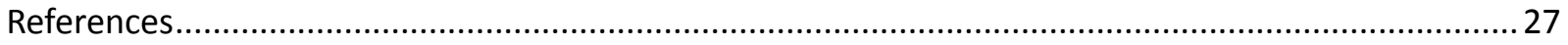

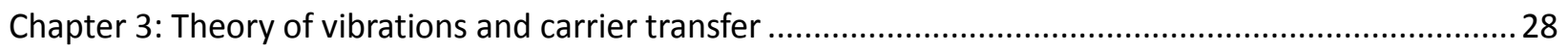

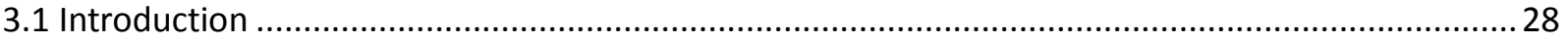

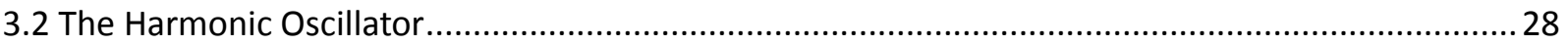

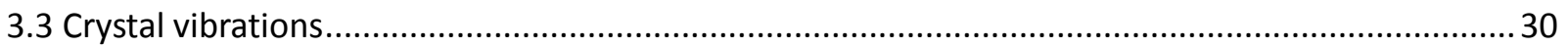

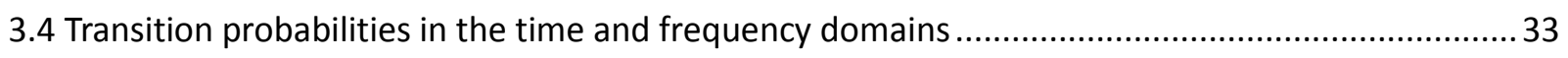

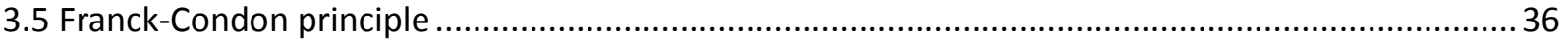

3.6 Classical Marcus theory of electron transfer ................................................................................ 41

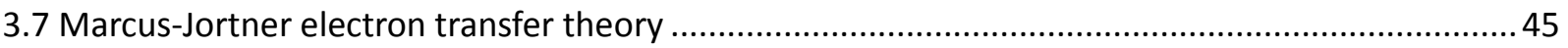




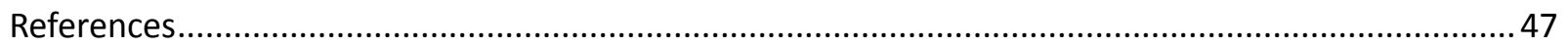

Chapter 4: Surface trapping in semiconductor nanocrystals.............................................................. 48

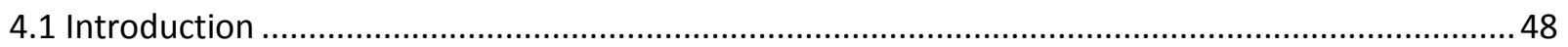

4.2 Temperature-dependent emission from semiconductor nanocrystals ........................................ 48

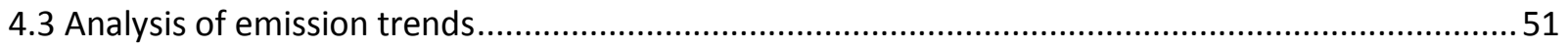

4.4 Theoretical approaches to understanding surface trapping …................................................... 53

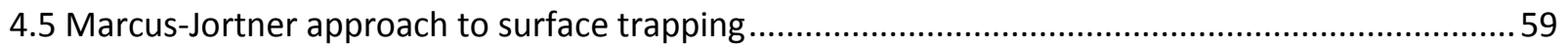

4.6 Marcus-Jortner electron transfer: a more complete picture ..................................................... 64

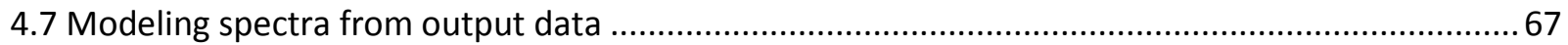

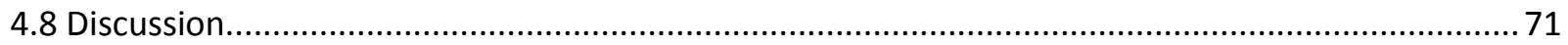

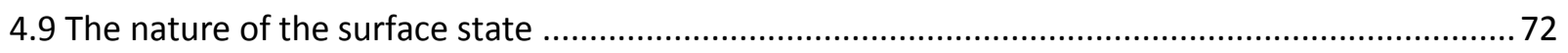

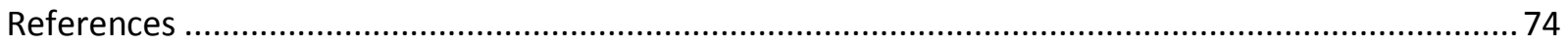

Chapter 5: Coupling strength and hot carrier effects .......................................................................... 77

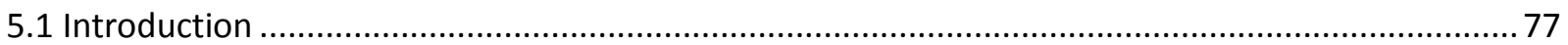

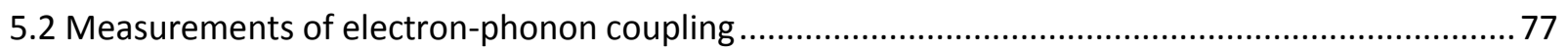

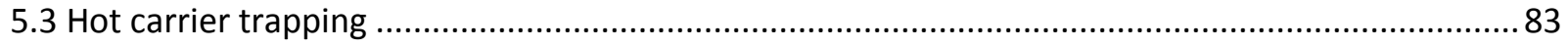

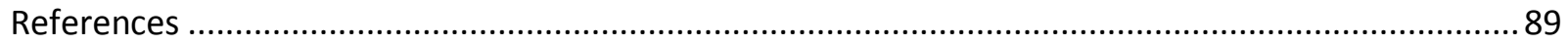

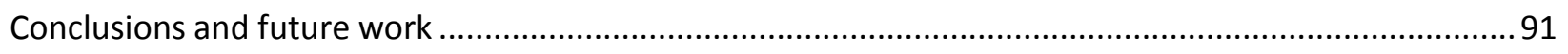

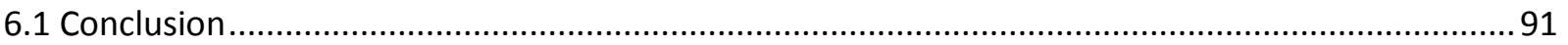

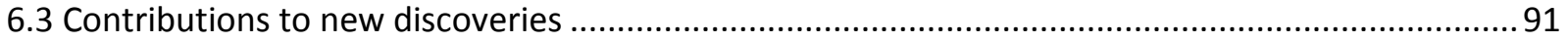

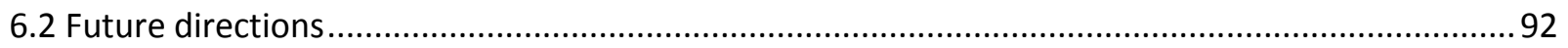

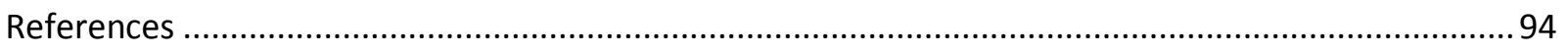

Appendix A …

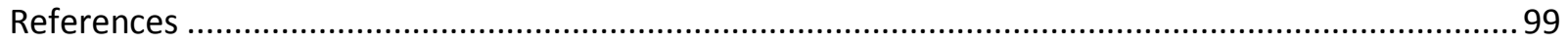

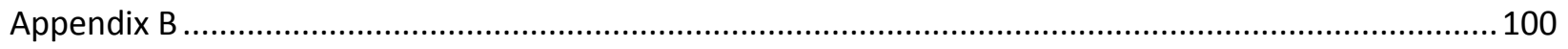

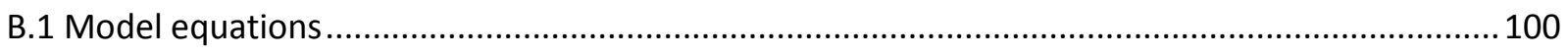

B.1. Treatment of the temperature-dependence of the quantum yield ............................................ 101

B.2. Treatment of reverse electron transfer rate ........................................................................... 104

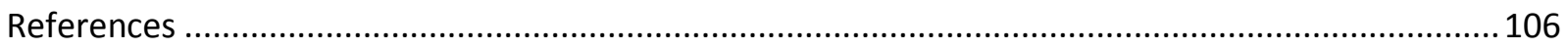




\section{Acknowledgements}

First, I thank Michael Krause for many years of fruitful and gratifying scientific collaboration and friendship. Officer Krause was an ideal and very understanding colleague to work with. I fondly recall spending numerous late nights together transforming findings which didn't yield any obviously meaningful insights into manuscripts that would earn acceptance in well-regarded and peer-reviewed scholarly journals. I also thank him for many afternoons and evenings spent drinking together that lead to the scientific ideas and revelations that oddly enough form the basis for actual experiments being performed in the Kambhampati group.

I thank blue monster Pooja Tyagi for helpful discussions in improving this thesis, for bringing endless entertainment to the Kambhampati group, and for stimulating discussions about the reaction coordinate.

I thank Jonathan Saari for his major contribution to the experimental work on phonons that is partially presented in Chapter 5 .

I thank Timothy Mack for carefully reading drafts of this thesis and making helpful suggestions and for bringing new and refreshing insights to the Kambhampati surface subgroup.

I thank Kunal Tiwari for offering excellent suggestions to address gaps and shortcomings in drafts of this thesis.

I thank Samuel Palato for suggesting several important improvements to this thesis that improved the clarity of several sections and addressed some shortcomings.

I thank Brenna Walsh for useful suggestions on drafts of this thesis and helpful comments that allowed several sections of this thesis to be clarified and improved. 
I thank Hélène Seiler for unexpectedly intense but stimulating discussions regarding the appropriate use of the principle of conservation of energy.

I thank Fred Kluck, J.P. Guay, Rick Rossi, Olivia Dinica, and Klaudia Jumaa for critical support with experiments.

I thank McGill University and Fonds québécois de la recherche sur la nature et les technologies for financial support.

I thank Chantal Marotte for consistent support and sympathy throughout my graduate student career.

I thank Samuel Sewall for serving as a mentor during my early work in the Kambhamapti group and the serving as doyen of the group.

Finally, I thank Patanjali Kambhampati for the great deal that I learned about scientific communication in the process of writing manuscripts with him, for serving as a source of motivation, encouragement, constructive criticism, and support (and the occasional joke among his students), for providing a flexible environment that enabled students to embark on scientific and personal pursuits, and for keeping at the forefront of the field and cultivating his group's and my own engagement with the scientific community. 


\section{Preface and contribution of authors}

The original contribution of this thesis is to offer a model of charge trapping to the surface of a nanocrystal that explains both the unique thermal response and the unusual spectral properties of surface emission in semiconductor nanocrysatals. This model, introduced in Chapter 3, holds that surface trapping is a semiclassical electron-transfer process in which the polarization induced by the trapped charged yields a surface state with strong electron-phonon coupling. This strong coupling, in turn, is responsible for the unique breadth and low energy of the surface emission. In addition, population exchange via trapping to and detrapping from the surface occurs readily, allowing the relative population of surface-trapped charge carriers to be modulated by temperature. Using parameters obtained from this model, the full nanocrystal emission spectrum, including the surface emission band, is accurately reproduced at several temperatures via simulations using the wavepacket correlation function approach. Finally, in Chapter 4 the foundation for extending this model to treat hot (excess energy) exciton trapping is presented.

Regarding the work on surface trapping presented in Chapters 4 and 5, Jonathan Mooney and Michael M. Krause performed the experiments, and Jonathan Mooney, Michael M. Krause, and Patanjali Kambhampati, and Jonathan I. Saari analyzed the data, while Jonathan Mooney and Patanjali Kambhampati wrote the manuscripts.

Regarding the work on phonons presented in Chapter 5, Jonathan I. Saari, Jonathan Mooney, Michael M. Krause, and Brenna R. Walsh performed the experiments, Jonathan Mooney, Jonathan I. Saari, and Patanjali Kambhampati analyzed the data, and Patanjali Kambhampati, Jonathan Mooney, and Anne Myers Kelley wrote the manuscript. 
Regarding the work on hot exciton trapping presented in Chapter 6, Jonathan Mooney, Michael M. Krause, and Jonathan I. Saari, performed the experiments, Jonathan Mooney, Jonathan I. Saari, Michael M. Krause and Patanjali Kambhampati analyzed the data, and Jonathan Mooney and Patanjali Kambhampati wrote the manuscript. 


\section{Chapter 1: Introduction}

\subsection{Summary}

Semiconductor nanocrystals are solids in which the lattice contains only hundreds of atoms. As a result of their nanometer size, nanocrystals' properties and behaviour are dictated by quantum effects, making them a model system in which to explore quantum mechanics. In addition, nanocrystals have found applications as fluorescent biological labels, sensors, and optical devices.

The surface of the semiconductor nanocrystal plays a vital role in determining its properties, behaviour and potential for use in applications. For example, charge carriers can trap to states on the nanocrystal surface and radiatively recombine from these states over a broad energy range. Yet a comprehensive and consistent explanation of the nature of these surface states has yet to be offered. The prevailing view is that the large width and low energy of the surface emission band arises from a broad energetic distribution of emitting states that lie deep inside the band gap (Figure 1.1). But this view is inconsistent with experiments suggesting thermal exchange occurs between the surface and core states of the nanocrystal, which is energetically not possible for states deep inside the band gap. In this thesis, surface trapping is explained as a semiclassical electron-transfer process in which the trapped charge polarization produces strong electron-phonon coupling, leading to emission which is broad and redshifted due to a phonon progression. By providing a microscopic explanation of surface charge trapping that is consistent with all experimental data, this model presents a conceptual framework to guide 
chemical control of the nanocrystal surface for applications such as charge transport, sensing, or photochemistry.

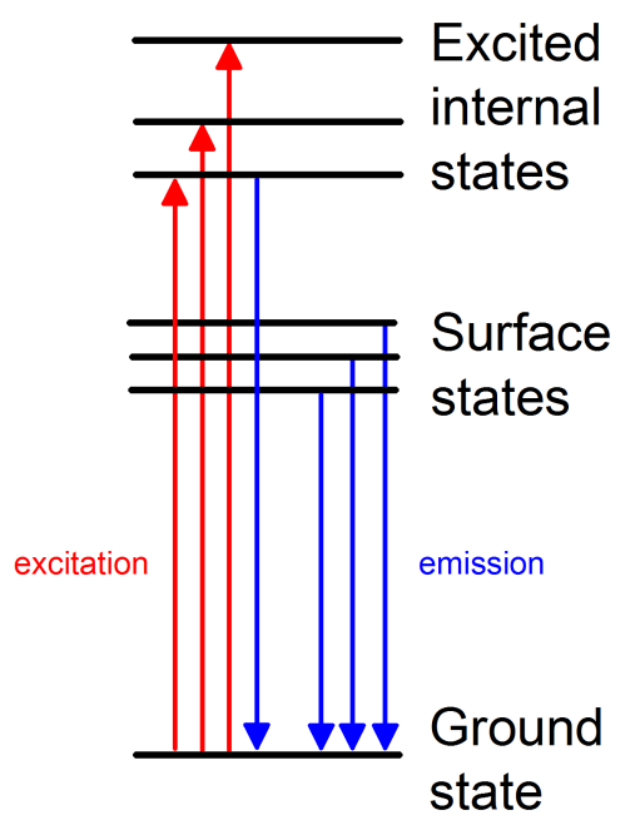

Figure 1.1. In addition to recombining radiatively from the lowest-energy "internal" excited state, charge carriers may trap at and recombine from surface states. In the traditional model, this energetic breadth of surface recombination is attributed to the existence of an energetically broad distribution of surface states.

\subsection{Motivation}

Since their emergence in the 1980s, semiconductor nanocrystals (NC) have attracted wide attention due to their potential applications as biological labels ${ }^{1}$ and light harvesters ${ }^{2}$ as well as 
their status as a model system for exploring quantum size effects. ${ }^{3}$ Increasingly, the surface of the nanocrystal is identified as playing a critical role in its properties and applications.

The surface of NCs is recognized for its importance in processes such as blinking ${ }^{4,5}$, optical gain $^{6-8}$, exciton relaxation dynamics ${ }^{6,9-11}$, and the conditions that determine quantum yield, ${ }^{12,13}$ photoluminescence lifetime, ${ }^{9}$ and the nature of the luminescence spectrum itself. ${ }^{14}$ Surface properties are especially important in small NCs given that a significant proportion of the atoms that compose these NCs are surface atoms.

The surface of the NC has been shown to play an especially critical role in whether potential NC applications are functional or not. Recent work has shown that surface traps play a critical role in the conduction efficiency of optoelectronic devices ${ }^{15}$ and that NC surface chemistry significantly affects Förster Resonance Energy Transfer to bioconjugates, highlighting the important role of the surface in sensing applications. ${ }^{16}$

It is widely accepted that charge carriers in NCs can sometimes become trapped on the NC surface $e^{10,17,18}$, yet the nature of this surface trapping remains poorly understood. A proper understanding of surface trapping in semiconducting nanocrystals holds promise for utilizing the surface in applications such as optoelectronic devices. ${ }^{19}$

\subsection{History}

Despite decades of study, the surface of the NC remains poorly understood relative to our understanding of the quantized excitonic states of the core of the NC. ${ }^{6,11,12}$ While the excitonic 
states are well described by the effective mass approximation applied to the particle-in-a-sphere model (see Chapter 2), this model assumes an infinite potential barrier at the surface of the nanocrystal. Yet it is well-known that carriers can escape from the core of the nanocrystal, suggesting that some refinement is required to this model that takes account of the nanocrystal surface.

NC surfaces in colloidal samples are typically passivated by organic molecules which bind to surface atoms. Initial work on the surface of NCs focused on the effect of different passivations on luminescence properties. The standard means to optically investigate NCs is to excite the system with a continuous-wave (CW) light source of energy equal to or greater than the band gap. The system then emits fluorescence via recombination from the excitonic states and also sometimes from surface states (Figures 1.1 and 1.2). Kuno et al. found that ligand exchanges resulted in the appearance of additional emission at lower energy in the photoluminescence (PL) spectrum (Figure 1.2) and a marked decrease in quantum yield. ${ }^{12}$ Guyot-Sionnest showed that capping the surface of CdSe NCs with a higher bandgap ZnS layer dramatically increased quantum yield and eliminated this low energy emission. ${ }^{20}$ Several additional studies ${ }^{18,21}$ demonstrated that the low energy emission could be quenched or enhanced by ligand or solvent changes, suggesting that low energy emission arises from states at the surface of the NC.

Understanding the precise nature of these surface states would provide significant insight into the behaviour of nanocrystals, and the low energy emission ascribed to these states offers one of the simplest means to monitor and examine surface trapping. Some of the earliest investigations into low energy emission from NCs were made by the group of Horst Weller. Noting the quenching of fluorescence in CdS NCs by $\mathrm{Cd}^{2+}$ and methyl viologen, Weller 


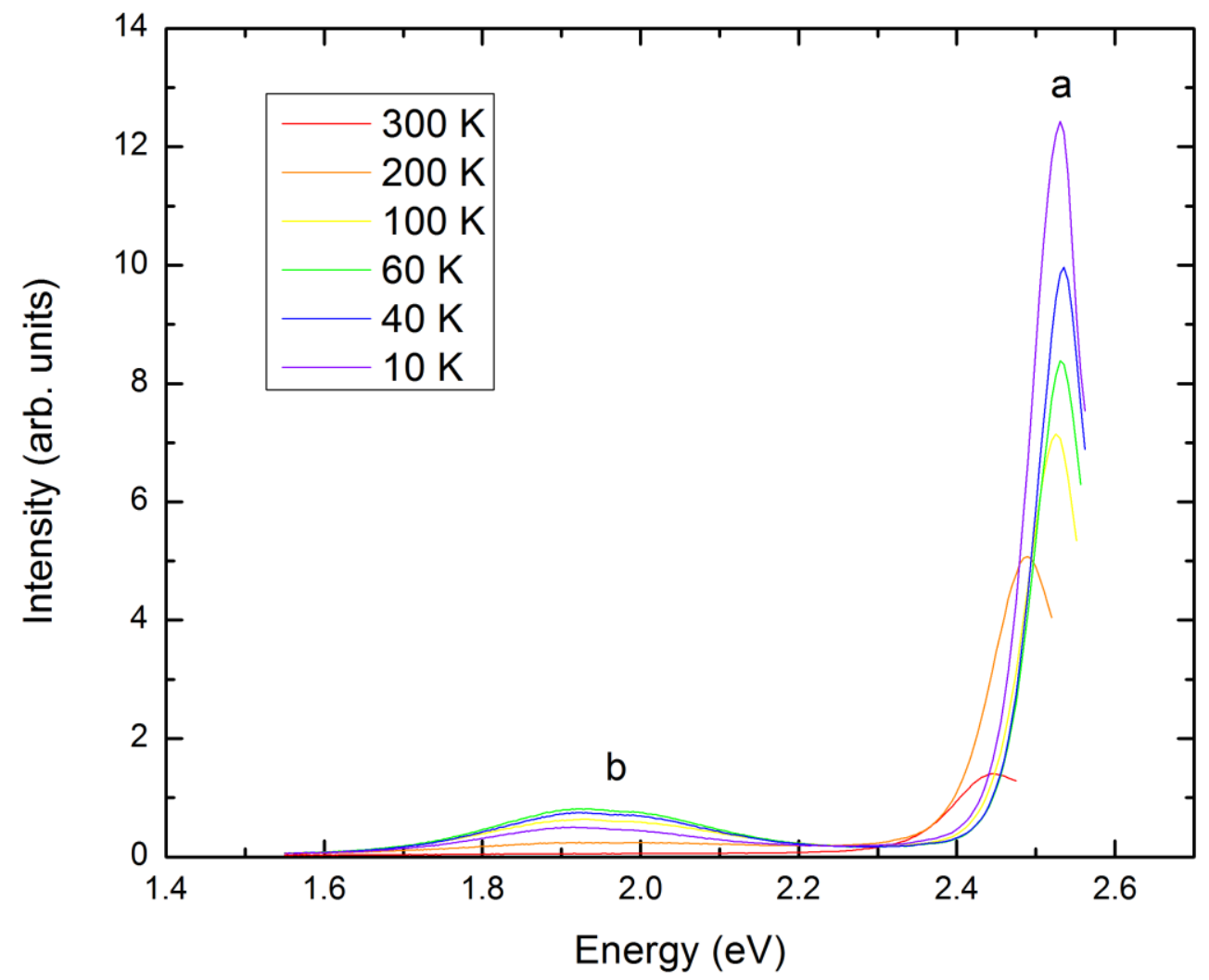

Figure 1.2 a) Temperature-dependent photoluminescence spectra of CdSe nanocrystals demonstrating excitonic (a) and surface (b) emission. The surface emission becomes significantly more visible at lower temperature.

suggested that this fluorescence occurs at surface anionic vacancies and that the broad emission occurs from electrons trapped in surface states of different energies. ${ }^{22,23}$ Further studies by Weller suggested that electrons were trapped in a narrow band while holes were trapped in a broad, deep distribution of states and argued, "Fluorescence spectroscopy is, therefore, an easy and sensitive method of studying the surface states of the particles. The spectral distribution gives information on the occurrence, population and depths of the surface traps." ${ }^{, 18}$ Findings 
from conductance spectroscopy ${ }^{24}$ and cyclic voltammetry ${ }^{25}$ experiments have been cited in support of this model, and the model proposed by Weller has been adopted by others. ${ }^{26}$

In the 1990s, theoretical work by Whaley's group found that an intense surface state with high oscillator strength could occur within the band gap of a CdSe nanocluster. These states would arise from unsaturated, "dangling" orbitals from Se surface atoms. ${ }^{27}$ Since these states are not observed experimentally, the authors suggested that surface relaxation might modify the energy of these states such that they move out of the band gap. A later study from the same group showed that while relaxation alone would not remove these states from the band gap, surface reconstruction together with a more different hybridization of the surface orbital would. ${ }^{28}$

The idea that surface states arise from "dangling" surface selenium atom orbitals is supported by experimental work from the Alivisatos and Bawendi groups. The Alivisatos group used X-ray photoelectron spectroscopy to investigate binding of ligand molecules to the nanocrystal surface. They found that while $\mathrm{Cd}$ surface atoms were well-passivated by trioctylphosphineoxide (TOPO) ligands, most surface Se atoms were completely unpassivated. ${ }^{29}$ The Bawendi group performed a ${ }^{31} P$ NMR study on TOPO-capped NCs. They found coverage ratios in good accord with the Alivisatos group and concluded nearly all $\mathrm{Cd}$ surface atoms are chemically passivated, while Se surface sites remain unpassivated. ${ }^{30}$

In 2001, a paper from Sandra Rosenthal's group provided significant insight into the nature of surface trapping and crystallized a model of understanding of the nature of surface emission in nanocrystals. Rosenthal's group found using fluorescence upconversion spectroscopy that the decay of excitonic emission was correlated with the rise of surface emission and developed a model for understanding the dynamics of surface trapping in NCs. 
Noting prior experimental and theoretical work indicating the presence of mid-gap selenium states in CdSe NCs and building on Weller's proposal, Rosenthal ascribed the breadth of the deep-trap emission to different species of Se dangling bonds on the surface of the NC. ${ }^{10}$ The model put forth in Rosenthal's work has been cited many times and forms the starting point for discussing the origin of surface emission in most of the recent literature. ${ }^{31-33}$

\subsection{The need for a new approach}

Several simple observations signal inconsistencies in this prevailing picture of the surface PL arising from mid-gap defect states. Temperature-dependent PL experiments reveal that the relative amount of surface PL reaches a maximum at intermediate temperature and exhibits a functional form distinct from the band edge core excitonic PL (Figure 1.2). ${ }^{34,35}$ Such behavior strongly suggests that the carrier exchange between the core state and the surface states is governed by forward and back activation barriers to population transfer. Forward and back barriers of appropriate magnitude would lead to a maximum in surface intensity at intermediate temperature and the unique functional form of the surface PL temperature-dependence. Yet the surface PL is redshifted from the core by $>10 \mathrm{k}_{\mathrm{B}} \mathrm{T}$; hence the assumed distribution of mid-gap defect states would not be capable of thermally induced carrier population (re)exchange at typical temperatures, in apparent contradiction to the observation.

Moreover, recent experimental work has shown that surface emission from an individual NC is inherently broad. ${ }^{36,37}$ Thus, according to the deep-trap model, the individual NCs would have to possess many trap states over a broad range of energy. As Banin first pointed out for nanorods $^{38}$, if this were the case then a redshifting of surface emission band would be expected at low temperature because there would be insufficient thermal energy to populate the higher- 
energy trap states in the NC. But no such redshifting is observed, demonstrating another contradiction between the deep-trap model and experiments.

This thesis challenges the prevailing view of nanocrystal surface emission as arising from a broad distribution of mid-gap defect states by examining temperature-dependent photoluminescence spectra of a range of NCs. The results of these temperature-dependent studies are fully explained by introducing a model which treats surface trapping as a semiclassical electron-transfer process that creates a strong polarization upon trapping. In this model, carriers can trap to and detrap from a single surface state with transition rates described by Marcus-Jortner electron transfer theory. Trapping to this surface state induces a strong polarization in the nanocrystal, resulting in strong electron-phonon coupling according to the Fröhlich mechanism.

The strong electron-phonon coupling that is created upon trapping explains the breadth and energy shift of the surface state emission. Emission from a state that is strongly coupled will take place in a Franck-Condon progression. The strength of the coupling is directly proportional to both the energy shift and the width of the emission. Perhaps most notably, the same values of coupling obtained from this model can account for both the electron-transfer rate and the spectral shape and energy of surface emission, a finding that is confirmed by simulating spectra based on a value of coupling obtained when fitting the temperature-dependent emission intensity data.

While prior works have suggested that electron-phonon coupling could be one factor determining the breadth of surface emission ${ }^{34,39}$ and invoked strong coupling to lattice phonons as part of a phenomenological explanation for the temperature-dependence, ${ }^{34}$ no work has emphasized the singularly important role of coupling in surface emission or provided a 
quantitative, microscopic model to explain the temperature-dependence of both core and surface emission.

The new approach offered in this thesis uniquely accounts for all observations and resolves the contradictions of the currently accepted model. In addition, these findings provide insight into the nature of the surface state, suggesting that is it is characterized by strong polarization and capable of thermal exchange with the core states of the NC. This model suggests that manipulation of the surface chemistry of NCs holds promise for energy harvesting and other applications via exploitation of this surface state.

\subsection{Thesis overview}

The electronic structure of semiconductor nanocrystals will be introduced in Chapter 2. The particle-in-a-sphere model with the effective mass approximation will be shown to be an effective theoretical treatment of nanocyrstal excitonic states, but the shortcomings of this model in treating surface states will be also considered. Chapter 3 will introduce the harmonic oscillator model for understanding vibrations and carrier transfer in nanocrystals. Using this model as a foundation, classical and semiclassical Marcus electron transfer theory will presented. These models permit the calculation of rates of electron transfer between states. In Chapter 4, semiclassical Marcus theory will be employed to explain the temperature-dependence of emission from the nanocrystal core and surface. The results of this treatment will be shown to also explain the spectrum of surface emission via Franck-Condon progressions from the harmonic oscillator model. Insights regarding the strong electron-phonon coupling in this surface state will be discussed. Chapter 5 will offer a more detailed treatment of phonons and coupling in NCs using continuous-wave and ultrafast spectroscopy and suggest that the model 
developed regarding trapping can also be employed to describe the trapping of "hot" (excess

energy) excitons. Finally, the conclusion will summarize major insights of this work and suggest

directions for future experiments.

\section{References}

1. Bruchez, M.; Moronne, M.; Gin, P.; Weiss, S.; Alivisatos, A. P., Semiconductor nanocrystals as fluorescent biological labels. Science 1998, 281, 2013-2016.

2. Kamat, P. V., Quantum Dot Solar Cells. Semiconductor Nanocrystals as Light Harvesters. J Phys Chem C 2008, 112, 18737-18753.

3. Alivisatos, A. P., Semiconductor clusters, nanocrystals, and quantum dots. Science 1996, 271, 933-937.

4. Galland, C.; Ghosh, Y.; Steinbruck, A.; Sykora, M.; Hollingsworth, J. A.; Klimov, V. I.; Htoon, H., Two types of luminescence blinking revealed by spectroelectrochemistry of single quantum dots. Nature 2011, 479, 203-U75.

5. Rosen, S.; Schwartz, O.; Oron, D., Transient Fluorescence of the Off State in Blinking $\mathrm{CdSe} / \mathrm{CdS} / \mathrm{ZnS}$ Semiconductor Nanocrystals Is Not Governed by Auger Recombination. Phys Rev Lett 2010, 104, 157404.

6. Kambhampati, P., Unraveling the Structure and Dynamics of Excitons in Semiconductor Quantum Dots. Acc Chem Res 2011, 44, 1-13.

7. $\quad$ Cooney, R. R.; Sewall, S. L.; Sagar, D. M.; Kambhampati, P., State-Resolved Manipulations of Optical Gain in Semiconductor Quantum Dots: Size Universality, Gain Tailoring, and Surface Effects. J Chem Phys 2009, 131, 164706.

8. Cooney, R. R.; Sewall, S. L.; Sagar, D. M.; Kambhampati, P., Gain Control in Semiconductor Quantum Dots via State-Resolved Optical Pumping. Phys Rev Lett 2009, 102, 127404-4.

9. $\quad$ Garrett, M. D.; Bowers, M. J.; McBride, J. R.; Orndorff, R. L.; Pennycook, S. J.; Rosenthal, S. J., Band edge dynamics in CdSe nanocrystals observed by ultrafast fluorescence upconversion. J Phys Chem C 2008, 112, 436-442.

10. Underwood, D. F.; Kippeny, T.; Rosenthal, S. J., Ultrafast Carrier Dynamics in CdSe Nanocrystals Determined by Femtosecond Fluorescence Upconversion Spectroscopy. J Phys Chem B 2001, 105, 436-443.

11. Kambhampati, P., Hot Exciton Relaxation Dynamics in Semiconductor Quantum Dots: Radiationless Transitions on the Nanoscale. J Phys Chem C 2011, 115, 22089-22109.

12. Kuno, M.; Lee, J. K.; Dabbousi, B. O.; Mikulec, F. V.; Bawendi, M. G., The band edge luminescence of surface modified CdSe nanocrystallites: Probing the luminescing state. $J$ Chem Phys 1997, 106, 9869-9882.

13. Asami, H.; Abe, Y.; Ohtsu, T.; Kamiya, I.; Hara, M., Surface state analysis of photobrightening in CdSe nanocrystal thin films. J Phys Chem B 2003, 107, 12566-12568.

14. Baker, D. R.; Kamat, P. V., Tuning the Emission of CdSe Quantum Dots by Controlled Trap Enhancement. Langmuir 2010, 26, 11272-11276. 
15. Geyer, S.; Porter, V. J.; Halpert, J. E.; Mentzel, T. S.; Kastner, M. A.; Bawendi, M. G., Charge transport in mixed CdSe and CdTe colloidal nanocrystal films. Phys Rev B 2010, 82, 155201.

16. Zhang, Y.; Zhang, H. Y.; Hollins, J.; Webb, M. E.; Zhou, D. J., Small-molecule ligands strongly affect the Forster resonance energy transfer between a quantum dot and a fluorescent protein. Phys Chem Chem Phys 2011, 13, 19427-19436.

17. Guyot-Sionnest, P.; Wehrenberg, B.; Yu, D., Intraband relaxation in CdSe nanocrystals and the strong influence of the surface ligands. J Chem Phys 2005, 123, 074709.

18. Hasselbarth, A.; Eychmuller, A.; Weller, H., Detection of Shallow Electron Traps in Quantum Sized Cds by Fluorescence Quenching Experiments. Chem Phys Lett 1993, 203, 271276.

19. Talapin, D. V.; Poznyak, S. K.; Gaponik, N. P.; Rogach, A. L.; Eychmuller, A., Synthesis of surface-modified colloidal semiconductor nanocrystals and study of photoinduced charge separation and transport in nanocrystal-polymer composites. Physica E 2002, 14, 237-241.

20. Hines, M. A.; Guyot-Sionnest, P., Synthesis and Characterization of Strongly Luminescing ZnS-Capped CdSe Nanocrystals. Journal of Physical Chemistry 1996, 100, 468-71.

21. Kalyuzhny, G.; Murray, R. W., Ligand effects on optical properties of CdSe nanocrystals. JPhys Chem B 2005, 109, 7012-7021.

22. Weller, H.; Koch, U.; Gutierrez, M.; Henglein, A., Photochemistry of Colloidal Metal Sulfides .7. Absorption and Fluorescence of Extremely Small Zns Particles - the World of the Neglected Dimensions. Ber Bunsen Phys Chem 1984, 88, 649-656.

23. Fojtik, A.; Weller, H.; Koch, U.; Henglein, A., Photo-Chemistry of Colloidal Metal Sulfides .8. Photo-Physics of Extremely Small Cds Particles - Q-State Cds and Magic Agglomeration Numbers. Ber Bunsen Phys Chem 1984, 88, 969-977.

24. Alperson, B.; Rubinstein, I.; Hodes, G., Identification of surface states on individual CdSe quantum, dots by room-temperature conductance spectroscopy. Phys Rev B 2001, 63 . 25. Kucur, E.; Bucking, W.; Giernoth, R.; Nann, T., Determination of defect states in semiconductor nanocrystals by cyclic voltammetry. J Phys Chem B 2005, 109, 20355-20360.

26. Hoheisel, W.; Colvin, V. L.; Johnson, C. S.; Alivisatos, A. P., Threshold for QuasiContinuum Absorption and Reduced Luminescence Efficiency in Cdse Nanocrystals. J Chem Phys 1994, 101, 8455-8460.

27. Hill, N. A.; Whaley, K. B., A Theoretical-Study of the Influence of the Surface on the Electronic-Structure of Cdse Nanoclusters. J Chem Phys 1994, 100, 2831-2837.

28. Pokrant, S.; Whaley, K. B., Tight-binding studies of surface effects on electronic structure of CdSe nanocrystals: the role of organic ligands, surface reconstruction, and inorganic capping shells. Eur Phys J D 1999, 6, 255-267.

29. Katari, J. E. B.; Colvin, V. L.; Alivisatos, A. P., X-Ray Photoelectron-Spectroscopy of Cdse Nanocrystals with Applications to Studies of the Nanocrystal Surface. J Phys Chem-Us 1994, 98, 4109-4117.

30. Becerra, L. R.; Murray, C. B.; Griffin, R. G.; Bawendi, M. G., Investigation of the Surface-Morphology of Capped Cdse Nanocrystallites by P-31 Nuclear-Magnetic-Resonance. $J$ Chem Phys 1994, 100, 3297-3300.

31. Bowers, M. J.; McBride, J. R.; Rosenthal, S. J., White-light emission from magic-sized cadmium selenide nanocrystals. J Am Chem Soc 2005, 127, 15378-15379.

32. McBride, J. R.; Dukes, A. D.; Schreuder, M. A.; Rosenthal, S. J., On ultrasmall nanocrystals. Chem Phys Lett 2010, 498, 1-9. 
33. Babentsov, V.; Riegler, J.; Schneider, J.; Fiederle, M.; Nann, T., Excitation dependence of steady-state photoluminescence in CdSe nanocrystal films. J Phys Chem B 2005, 109, 1534915354.

34. Lifshitz, E.; Dag, I.; Litvin, I.; Hodes, G.; Gorer, S.; Reisfeld, R.; Zelner, M.; Minti, H., Properties of CdSe nanoparticle films prepared by chemical deposition and sol-gel methods. Chem Phys Lett 1998, 288, 188-196.

35. Babentsov, V.; Sizov, F., Defects in quantum dots of IIB-VI semiconductors. OptoElectronics Review 2008, 16, 208-225.

36. Dukes, A. D.; Samson, P. C.; Keene, J. D.; Davis, L. M.; Wikswo, J. P.; Rosenthal, S. J., Single-Nanocrystal Spectroscopy of White-Light-Emitting CdSe Nanocrystals. J Phys Chem A 2011, 115, 4076-4081.

37. Layek, A.; De, S.; Thorat, R.; Chowdhury, A., Spectrally Resolved Photoluminescence Imaging of ZnO Nanocrystals at Single-Particle Levels. J Phys Chem Lett 2011, 2, 1241-1247. 38. Kan, S. H.; Aharoni, A.; Mokari, T.; Banin, U., Shape control of III-V semiconductor nanocrystals: Synthesis and properties of InAs quantum rods. Faraday Discuss 2004, 125, 23-38. 39. Chestnoy, N.; Harris, T. D.; Hull, R.; Brus, L. E., Luminescence and Photophysics of Cds Semiconductor Clusters - the Nature of the Emitting Electronic State. Journal of Physical Chemistry 1986, 90, 3393-3399. 


\section{Chapter 2: Electronic structure of semiconductor nanocrystals}

\subsection{Introduction}

This chapter examines the theoretical treatment of nanocrystal electronic structure. After reviewing traditional approaches to bulk semiconductors, the standard nanocrystal particle-in-asphere model employing the effective mass approximation is introduced. Effects specific to cadmium selenide are considered, and the fine structure that emerges in this nanoscale system is discussed. Finally, the shortcomings of this model in accounting for the nanocrystal surface are described and a brief review of theoretical works on nanocrystal surface states is presented.

\subsection{Semiconductors from bulk to nanoscale}

In solid state physics, the starting point for understanding crystals is the free electron model, which provides a simplified account of the behaviour of valence electrons in a periodic lattice. In this model, the electron is confined to a cube with sides of length $\mathrm{L}$ and the potential created by the atoms in the lattice is ignored. Under the Born-von Karman boundary conditions, the wavefunction is held to be periodic over lengths $L$, mimicking an infinite lattice. ${ }^{1}$ The quantum mechanical treatment of this system results in the following energy eigenvalues for the electron:

$$
E_{k}=\frac{\hbar^{2}}{2 m} k^{2}
$$

with $\hbar$ as the reduced Planck constant, $m$ as the mass of the electron and wavevector $k=0$, $\pm 2 \pi / L, \pm 4 \pi / L$, etc. due to the cube length and the periodic boundary conditions. Given that a bulk material is being treated, the macroscopic value of $L$ results in a very small spacing between energy levels, leading to a so-called quasi-continuous distribution of energy values. For 
example, if $L$ is set at $1 \mathrm{~m}$, the spacings between energy levels are $\sim 10^{-37} \mathrm{~J}$. The upper half of Figure 2.1a shows the dispersion curve obtained using the free-electron model.

An improvement on the free electron model can be made by introducing a weak, periodic potential to account for the effect of atoms on the electrons. This perturbation lifts the energy degeneracy at the Brillouin zone boundaries, introducing the characteristic band gap. ${ }^{1}$ Notably, in the reduced Brillouin zone representation, the resulting gap will occur at $k=0$. This simple model accounts for much of the fundamental physics of bulk semiconductors.
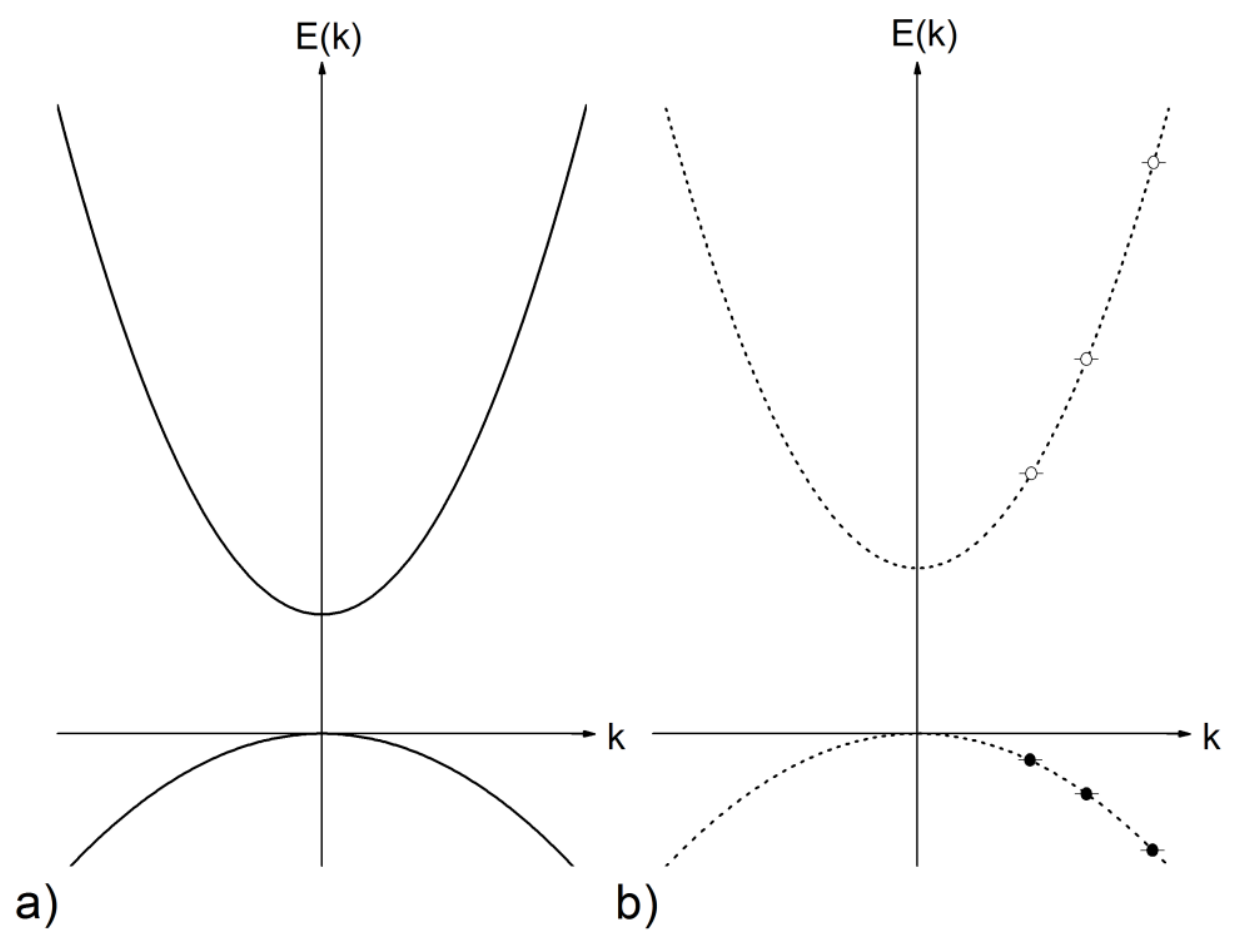

b)

Figure 2.1 a) Dispersion curves for bulk semiconductor (a) and nanocrystal (b) in the free electron model. The curves for the nanocrystal include discrete transitions and the band gap is larger due to the confinement energy.

To treat a nanocrystal system, the free-electron model is used, but rather than being confined to a cube, the electron is subject to zero potential within a sphere of radius $R$ and 
infinite potential elsewhere. In this case, solving the Schrödinger equation yields the following wavefuctions:

$$
\Phi_{n, l, m}(r, \theta, \phi)=C \frac{j_{l}\left(k_{l, n} r\right) Y_{l}^{m}(\theta, \phi)}{r}
$$

where $\mathrm{C}$ is a normalization constant, $Y_{l}^{m}(\theta, \phi)$ is a spherical harmonic, and $j_{l}\left(k_{l, n} r\right)$ is the $l^{\text {th }}$ order spherical Bessel function. The corresponding energies are:

$$
E_{k}=\frac{\hbar^{2}}{2 m} k_{n, l}^{2}
$$

Here $\mathrm{k}_{n, l}=\alpha_{n, l} / \mathrm{R}$, where $\alpha_{\mathrm{n}, l}$ is the $\mathrm{n}^{\text {th }}$ root of the $l^{\text {th }}$ order spherical Bessel function (the four smallest ones are $\alpha_{0,1}=\pi ., \alpha_{1,1}=4.49, \alpha_{2,1}=5.76$, and $\left.\alpha_{0,2}=2 \pi\right) .{ }^{2}$ The states are labeled using the quantum numbers $\mathrm{n}(1,2,3 \ldots)$ and $\mathrm{l}(\mathrm{s}, \mathrm{p}, \mathrm{d} \ldots)$ as $n L$ (e.g. 1S). As in the bulk case, energy spacing is inversely proportional to a length characteristic to the system, but in contrast to the bulk case values of $R$ are on the nanoscale, resulting in clearly quantized energy states (Figure 2.1b). For example, assuming a radius of $1 \mathrm{~nm}$ yields an $l=0$ to $l=1$ spacing of $10^{-19} \mathrm{~J}$, on the same order as the electronvolt.

Obviously this treatment has still completely ignored the effect of the lattice atoms by assuming an unreasonable potential of uniformly zero within the sphere. As a first refinement, the potential introduced by the atoms can be incorporated by treating the carriers (electrons and holes) as if they behave with an effective mass, $\mathrm{m}_{\text {eff }}$ different from their actual mass due to the presence of these atoms. ${ }^{2}$ This effective mass, by definition, is proportional to the second derivative of the dispersion curve: 


$$
m_{e f f}=\hbar^{2} \frac{\partial^{2} E}{\partial k^{2}}
$$

Thus, introducing an effective mass adjusts the curvature of the dispersion curve parabola. Notably, the effective mass can become negative near the top of a dispersion curve as a band fills up with electrons (Figure 2.1). In this configuration, absences of electrons are considered the charge carriers themselves and are referred to as holes. ${ }^{3}$ In the effective mass treatment, the dispersion curves are still assumed to be parabolic near band extrema, resulting in the following expressions for the conduction and valence bands in semiconductors:

$$
\begin{gathered}
E_{k}^{c}=\frac{\hbar^{2} k^{2}}{2 m_{e f f}^{c}}+E_{\text {bulk band gap }} \\
E_{k}^{v}=\frac{\hbar^{2} k^{2}}{2 m_{e f f}^{v}}
\end{gathered}
$$

Equations 2.5 and 2.6 account for the shape of the bands shown in Figure 2.1. A further refinement to this model involves taking the Coulombic attraction between the electron and the hole into account. Since this interaction scales as $1 / R$ while the carrier energy scales as $1 / R^{2}$, for sufficiently small crystallites, the confinement term dominates and the electrons and holes can be treated as independent particles with the Coulombic attraction term simply added as a first-order correction to the energy. Thus, the improved energy expression for carriers when including these corrections is: 


$$
E=E_{\text {bulk band gap }}+\frac{\hbar^{2}}{2 R^{2}}\left(\frac{\alpha_{\mathrm{n}, \mathrm{l}}^{\text {hole }}}{m_{\text {eff }}^{v}}+\frac{\alpha_{\mathrm{n}, \mathrm{l}}^{\text {electron }}}{m_{\text {eff }}^{c}}\right)-E_{\text {Coulombic }}
$$

here $\alpha_{\mathrm{n}, 1}$ is the Bessel function root as defined following equation 2.3. The complete equation 2.7 accounts for the band structure show in Figure 2.1.

The second confinement term illustrates the size-tunability of the band gap in nanocrystals. Due to the very small values of $R$, this term has an appreciable influence on the energy, so adjusting the value of $R$ allows the band gap energy to be modulated. Due to the smaller effective mass of electrons, the conduction band energy is primarily affected by changes in nanocrystal size.

\subsection{Cadmium selenide}

Cadmium selenide (CdSe) is a direct-gap II-IV semiconductor (i.e. the valence and conduction band extrema occur at the same value of $k$ ). It possesses a bulk band gap of $1.8 \mathrm{eV}$ and a wurtzite crystal structure. The conduction band arises from the $\mathrm{Cd} 5 \mathrm{~s}$ orbitals and is twofold degenerate at $k=0$, including spin. The valence band arises from the Se $4 \mathrm{p}$ orbitals and is sixfold degenerate at $k=0$, including spin. While the conduction band is fairly well-described within the particle in a sphere model and the effective mass approximation, the sixfold degeneracy of the valence band requires some additional consideration.

The total angular momentum of the valence band p-like states is $J=l+s$, where $l$ refers to orbital angular momentum and $s$ is the spin angular momentum. The orbital angular momentum $l=1$ for a $\mathrm{p}$ state, but its projection $\mathrm{m}_{1}$ can take on values of 0 and \pm 1 , while $s$ can 
take on values of $\pm 1 / 2$, resulting in the sixfold degeneracy. A strong spin-orbit interaction due to the high atomic number of $\operatorname{silicon}^{4}(0.42 \mathrm{eV}$ at $k=0)$ lifts the degeneracy at $k=0$, producing two states in which only the total angular momentum $J=l+s$ is conserved. This total angular momentum takes on values from $|l-s|$ to $l+s$, thus splitting the valence band at $k=0$ into two bands with total angular momentum $J=3 / 2$ and $J=1 / 2 .^{2}$ The $J=3 / 2$ state is fourfold degenerate with angular momentum projections $J_{z}= \pm 3 / 2$ and $\pm 1 / 2$, while the $J=1 / 2$ state is twofold degenerate with angular momentum projections $J_{z}= \pm 1 / 2$. This lower, twofold degenerate state is called the split-off hole.

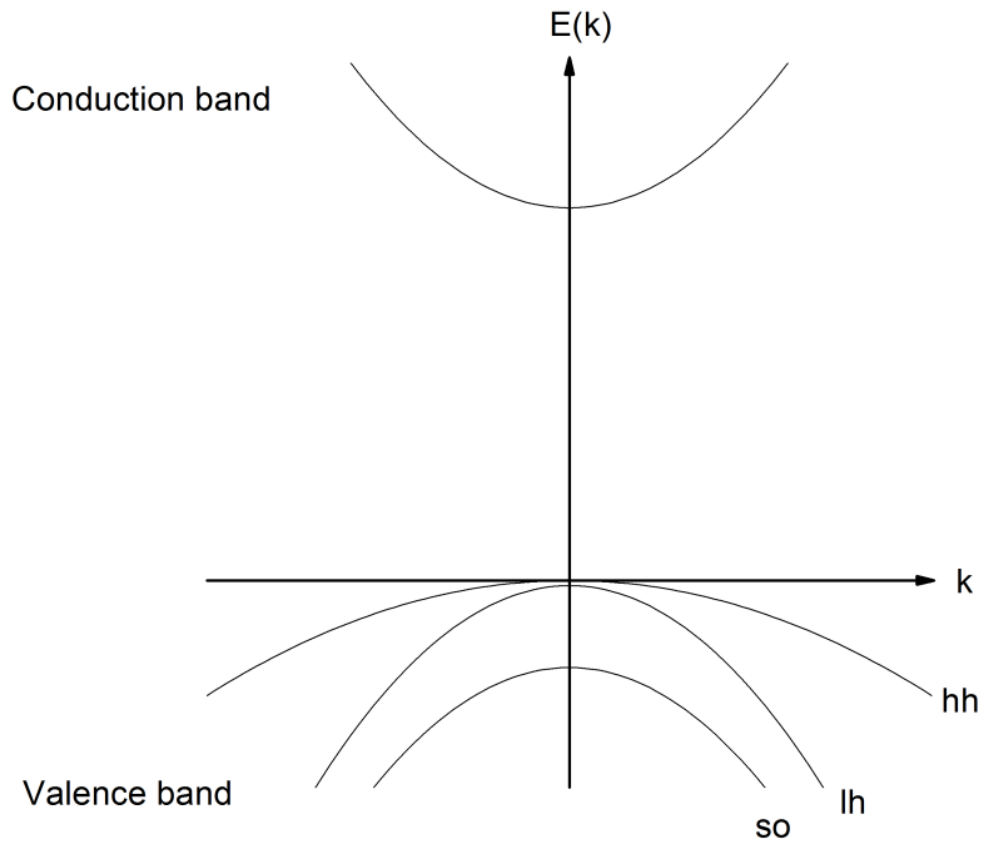

Figure 2.2 Dispersion curve for CdSe, including fine structure effects. Strong spin-orbit coupling lifts the degeneracy of the valence band, leading to the split-off hole (so) state. The Hamiltonian of the system results in two effective masses for the remaining band, the heavy hole (hh) and the light hole (lh), which are themselves split at $\mathrm{k}=0$ by the unique crystal axis of $\mathrm{CdSe}$ and nonsphericity effects. 
When a Hamiltonian including angular momentum is constructed and applied to the $J=$ $3 / 2$ state away from $k=0$, two energy eigenvalues result, with effective masses $m_{h}$ and $m_{l}$, called

the heavy hole and the light hole. ${ }^{5}$ The heavy hole has an angular momentum projection of $\pm 3 / 2$, while the light hole has a projection of $\pm 1 / 2$. In addition the wurtzite lattice in CdSe with its unique $\mathrm{c}$ axis produces a small $(25 \mathrm{meV})$ crystal field splitting, which lifts the degeneracy of the heavy hole and light hole bands even at $\mathrm{k}=0$. The three subbands that emerge from this treatment are referred to as the heavy hole, the light hole, and the split-off hole (Figure 2.2).

\subsection{Cadmium selenide on the nanoscale}

As mentioned previously, labeling of the states of nanocrystals involves the use of two quantum numbers that emerge from the particle-in-a-sphere treatment of the system, $n$ and $l$; states are thus labeled as $n L$ (e.g. 1S). However, the pseudo total angular momentum, $F$, of the state results from a combination of the particle-in-a-sphere angular momentum $L$ as well as a "unit cell" contribution to angular momentum arising from the original atomic basis of $\mathrm{CdSe}$ as described above and labeled $J$. The pseudo total angular momentum $F=L+J$, in analogy with $J=L+S$, takes on values from $|L-J|$ to $L+J . \quad$ The states are thus fully labeled $n L_{F}\left(\right.$ e.g. $\left.1 \mathrm{~S}_{3 / 2}\right)$. ${ }^{6,7}$

Ignoring the crystal field splitting from the wurtzite lattice described above, the first excited state of a CdSe nanocrystal is the eightfold degenerate $1 \mathrm{~S}_{\mathrm{e}}-1 \mathrm{~S}_{3 / 2}$ state, encompassing a twofold degenerate (from spin) conduction band $1 \mathrm{~S}_{\mathrm{e}}$ state and a fourfold degenerate valence band arising from the $J=3 / 2$ state. As mentioned above, the unique crystal axis actually splits the $J=3 / 2$ states into $J=3 / 2$ and $J=1 / 2$ projections, a splitting that is enhanced when effects of nonsphericity are included. Therefore, considering these effects only, the band edge exciton is split into two fourfold degenerate states (Figure 2.3). 
One very important effect which has been heretofore ignored is electron-hole exchange. In bulk semiconductors, this effect is marginal $(0.1 \mathrm{meV})$ whereas in organic molecules it is on the order of $1 \mathrm{eV}$. Since this term is proportional to the overlap between electrons and holes, in semiconductor nanocrystals the effect is enhanced due to carrier confinement. In the limit where the exchange term dominates, the important quantum number becomes system total angular momentum $\mathrm{F}$ which for the first excited state $\left(F_{h}=3 / 2, F_{e}=1 / 2\right)$ results in a fivefold degenerate $F$ $=2$ state (with projections $\pm 2, \pm 1$, and 0 ) and a threefold degenerate $F=1$ state (projections \pm 1 and 0$).^{2}$ When the exchange effect and the splitting are both included, the projections of $F$ become the good quantum numbers, resulting in five sublevels corresponding to the five projections, distinguished as lower (L) for the $F=2$ projections and upper (U) for the $F=1$ projections (Figure 2.3) ${ }^{8}$ Notably, emission from the lowest energy $\pm 2^{\mathrm{L}}$ states is optically forbidden by selection rules in the electric dipole approximation because two units of angular momentum are required to return to the ground state from this level. This state is labeled the dark exciton.

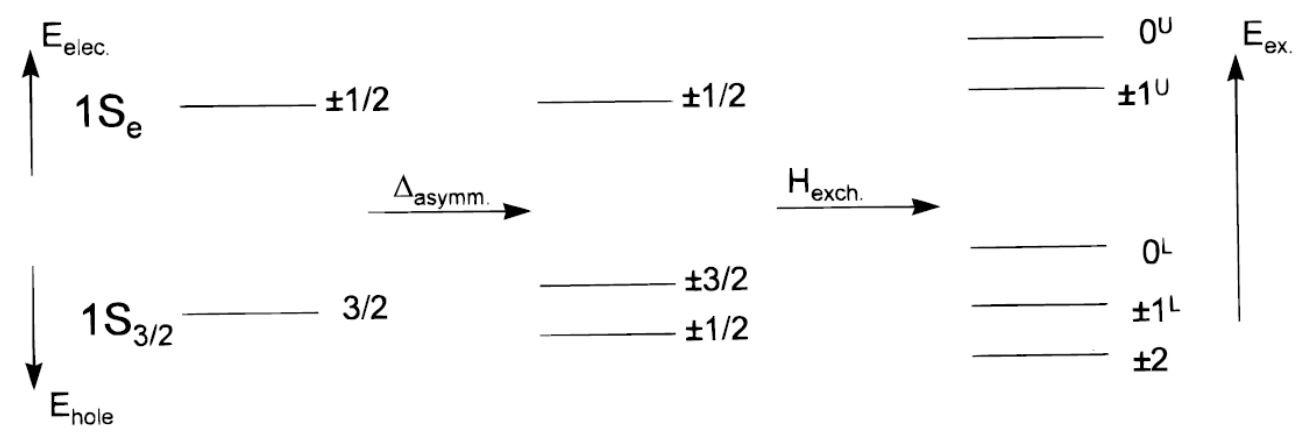

Figure 2.3 Asymmetry and electron-hole exchange lifts the degeneracy of the ground state in CdSe NCs, producing the fine structure. Reprinted with permission from "Luminescence Photophysics in Semiconductor Nanocrystals." Manoj Nirmal and Louis Brus. Accounts of Chemical Research 199932 (5), 407-414. Copyright (C) 1999 American Chemical Society 


\subsection{Nanocrystal synthesis and spectroscopy}

The typical method of preparing colloidal semiconductor nanocrystals involves a wet chemical synthesis that produces isolated nanocrystals dispersed in solvent (sols). The synthesis is designed as a precipitation reaction in which the nucleation period is short compared to the growth period, producing an ensemble of nanoparticles with low polydispersity. ${ }^{9}$ In this process, the size of the nanocrystal is controlled by the reaction time and other parameters such as temperature and the concentrations of reactants. During the growth phase, surfactant molecules in solution adsorb reversibly onto the surface of the nanocrystal, halting the growth and preventing individual nanocrystals from aggregating.

The selection rules for the probability of optical transitions can be determined simply when the electrons and holes are treated as independent of each other. This treatment is appropriate considering that the quadratic confinement term dominates the energy over the linear Coulombic term. The electron and hole wavefunctions, $\Psi_{\text {electron }}$ and $\Psi_{\text {electron }}$ respectively, which are based on those in equation 2.2, then appear as follows:

$$
\Psi_{\text {electron-hole pair }}=\Psi_{\text {electron }} \Psi_{\text {hole }}
$$

By the dipole matrix element, the transition probability is:

$$
P=\left|\left\langle\Psi_{\text {electron }}|\vec{e} \cdot \hat{p}| \Psi_{\text {hole }}\right\rangle\right|^{2}
$$

where $\vec{e}$ is the polarization vector of the light and $\hat{p}$ is the momentum operator.

Due to the orthonormality of the wavefunctions, the selection rules become $\Delta \mathrm{n}=0$ and $\Delta \mathrm{L}=0$. $^{2}$ These selection rules apply to transitions involving the creation or recombination of electron- 
hole pairs which are relevant to traditional nanocrystal spectroscopy. The low-energy, state to state "intraband" transitions involving single particles have different selection rules.

The absorption and emission spectra of a typical sample of CdSe nanocrystals is displayed in Figure 2.4. As is readily apparent from the absorption spectrum, discrete, state-like transitions are not visible. Rather, the transitions are broadened, appearing as bands rather than delta-function like discrete transitions. This broadening arises from the size dispersion of nanocrystals within the ensemble, in addition to homogeneous broadening of $\sim 50 \mathrm{meV} .^{10}$ Moreover, at higher energies, the density of conduction band states grows and state mixing makes individual transitions irresolvable. Nevertheless, at least the first two peaks in the absorption spectrum can be correlated with specific transitions between discrete excitonic states. As described above, the lowest-energy optical transition occurs between the $1 \mathrm{~S}_{\mathrm{e}}-1 \mathrm{~S}_{3 / 2}$ states and is highlighted in the spectrum. The second-lowest allowed transition occurs between the $1 \mathrm{~S}_{\mathrm{e}^{-}}$ $2 \mathrm{~S}_{3 / 2}$ states and is also indicated in the spectrum. Emission, which occurs from the lowest-energy excitonic state following Auger relaxation or energy transfer to ligands ${ }^{11}$, also appears as a single, inhomogeneously broadened band. 


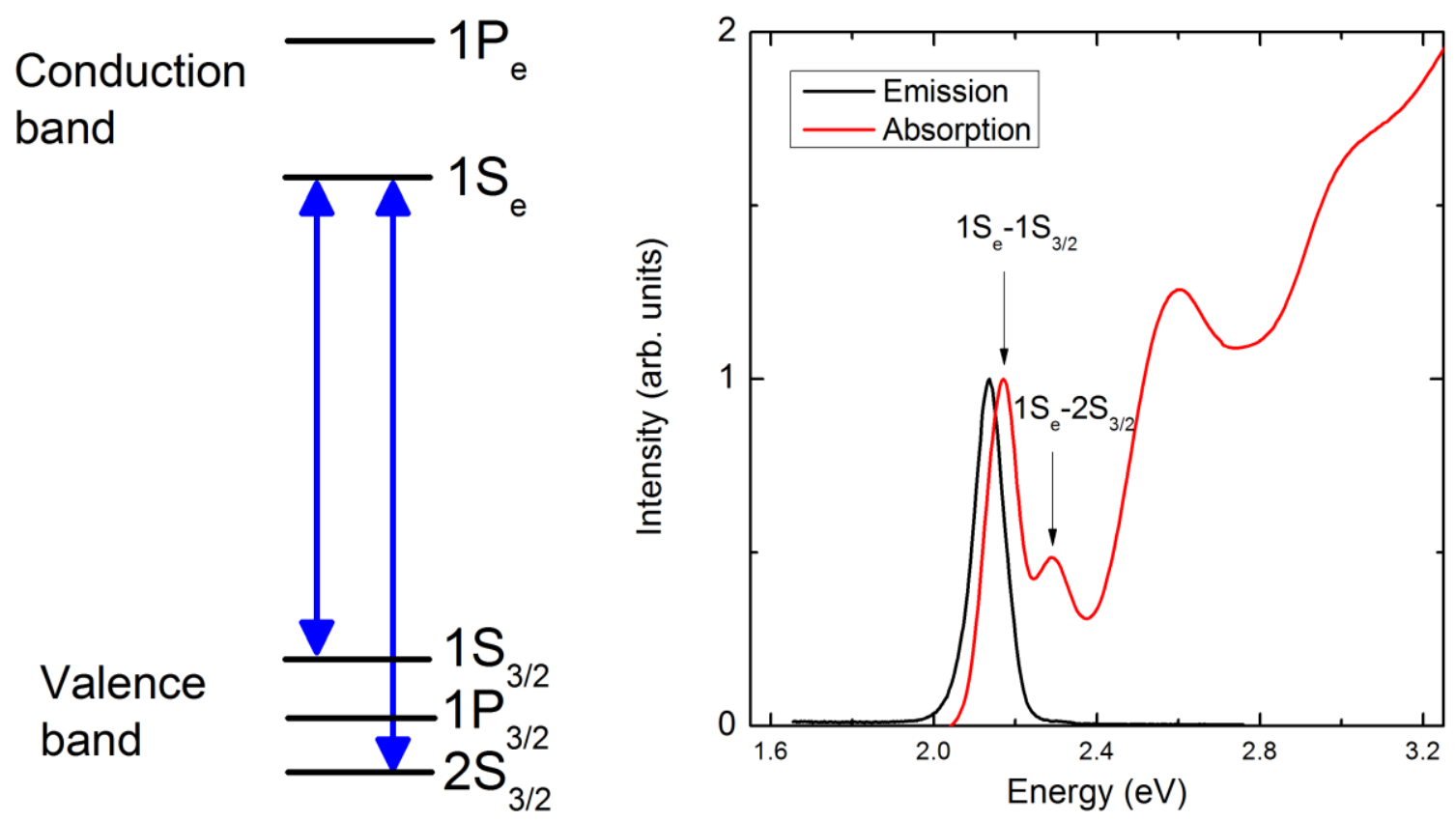

Figure 2.4. Absorption spectrum of CdSe nanocrystals. The first two transitions are highlighted and shown on the left as transitions in a schematic of the levels of the valence and conductions bands. The emission spectrum is also shown for comparison.

\subsection{The nanocrystal surface}

One of the clearest deficiencies of the particle-in-a-sphere model described above is assumption of an infinite potential barrier at the surface of the nanocrystal and the related Bornvon Karman (periodic) boundary condition, in which the wavefunction is assumed to simply "repeat" at the system boundaries. This problem also exists in the bulk semiconductor and a brief examination of its bulk treatment provides some insight into the issues at hand.

A preliminary accounting for the surface involves treating the system in one dimension and explicitly including the surface in the form of a boundary outside of which the potential is constant, rather than infinite. ${ }^{1}$ To properly consider this system, a more realistic potential than the 
free-electron case is typically used.

A typical treatment involves the assumption of a periodic potential within the crystal. This periodic potential terminates at the crystal surface, becoming constant there (Figure 2.5, solid line). Appropriate and physically reasonable wavefunctions are constructed on both sides of the interface based on these potentials; inside the crystal, this produces a periodic wavefunction (Figure 2.5, short dashed line), while outside it results in a smooth, exponentially decaying wavefunction.

The key insight in this treatment of surfaces is that the wavevector $k$ can in principle take on both real and imaginary values. ${ }^{12}$ Previously, in the absence of the surface, imaginary values were prohibited because they would have violated translational symmetry in the crystal, but they become possible in the presence of the surface. ${ }^{13}$ When an imaginary wavevector is employed on the crystal side of the interface, the resulting wavefunction exponentially decays inside the crystal (Figure 2.5, long dashed line) and is no longer delocalized. When the condition that wavefunctions on both sides of the interface as well as their derivatives be continuous at the interface is imposed and the system is solved in one dimension, the resulting wavefunctions are localized at the surface and have energies inside the bandgap (Figure 2.5, long dashed line). These solutions are called surface states. 


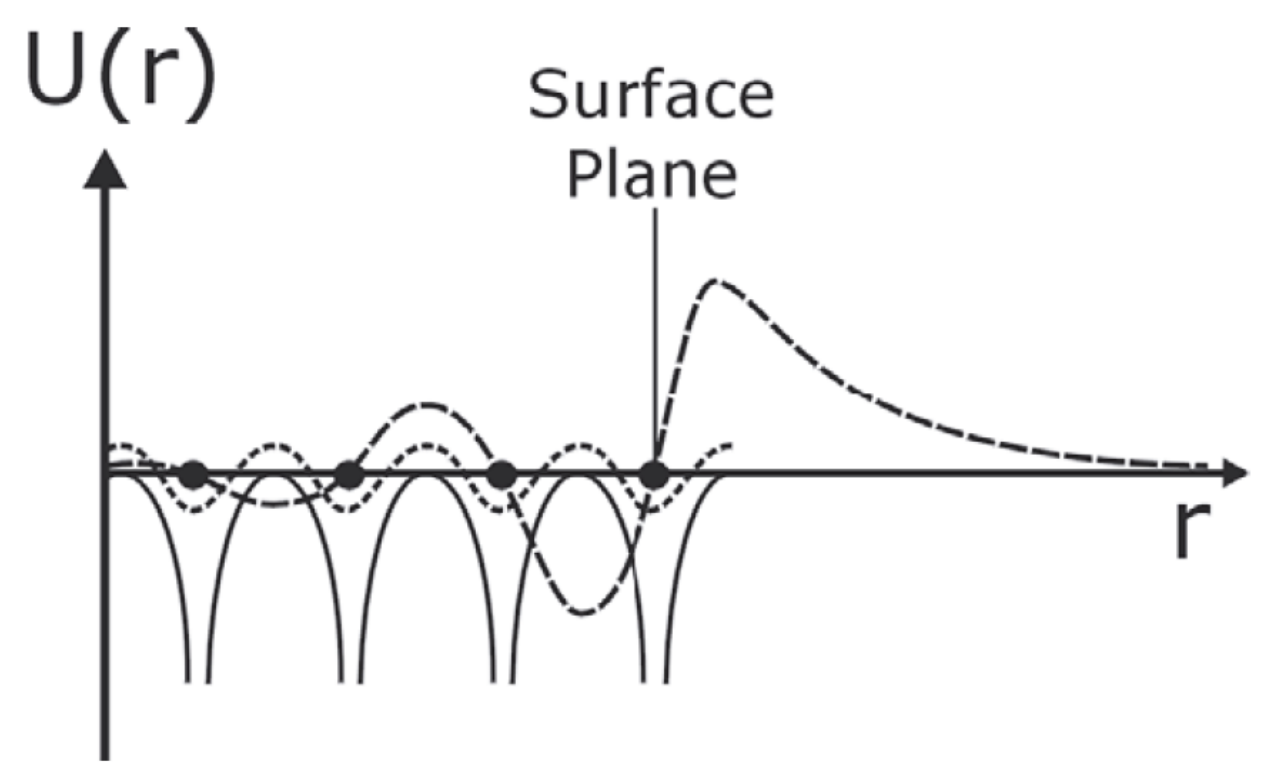

Figure 2.5 Surface state in a semiconductor. The potential is shown as a solid line, the normal wavefunction as a dotted line, and the surface state wavefunction as a dashed line. Figure 4 from Kevin M. Rosso, Structure and Reactivity of Semiconducting Mineral Surfaces: Convergence of Molecular Modeling and Experiment, Reviews in Mineralogy and Geochemistry, 2001, v. 42, p. $199-271$

Within the nanocrystal community, there have been several theoretical investigations regarding the presence of these surface states in nanocrystals. In 1994, Hill and Whaley conducted a theoretical study on trapping of charge carriers to surface states in CdSe NCs. ${ }^{14}$ Specifically, the authors calculated the transition probabilities for a CdSe system in which unpassivated or passivated surface states were explicitly included. Notably, they found that in clusters with Se-rich surfaces, an intense surface state appeared within the band gap. Such a state is not observed experimentally, a result the authors posited was due to surface relaxation which decreases the energy of the state toward the valence band. Nevertheless, the result is 
significant in that it shows that not only are mid-gap states possible, but also that surface states may exist and trap carriers without mid-gap states appearing in the absorption spectrum at all.

In 1999, Porkant and Whaley ${ }^{15}$ noted that experimental studies backed up their previous prediction of unpassivated Se atoms on the surface of the nanocrystal. They modeled the electronic structure of CdSe with prolate symmetry, examined the effect of passivation with TOPO ligands of different bond hybridization, predicting surface states within the band gap. Modeling which included surface reconstruction did not remove the Se orbitals within the gap, but including this effect and proposed different hybridization of the Se surface orbitals did. Again, this result is significant for showing that surface states may manifest in several ways in semiconductor nanocrystals.

In summary, the particle-in-a-sphere model with the effective-mass approximation provides a compelling and simple account of the primary features of semiconductor nanocrystals. However, the assumption of an infinite potential barrier represents an oversimplification. Surface states likely exist in nanocrystals and may remain underinvestigated due to the broad use of a model which does not account for them. 


\section{References}

1. Kittel, C., Introduction to solid state physics. 8th ed.; Wiley: Hoboken, NJ, 2005; p xix, $680 \mathrm{p}$.

2. Norris, D. J., Electronic Structure in Semiconductor Nanocrystals. In Semiconductor and metal nanocrystals : synthesis and electronic and optical properties, Marcel Dekker: New York, 2004; pp xiv, 484 p.

3. Ashcroft, N. W.; Mermin, N. D., Solid state physics. Holt: New York,, 1976; p xxi, 826 p.

4. Grundmann, M., The physics of semiconductors : an introduction including nanophysics and applications. 2nd ed.; Springer-Verlag: Berlin ; New York, 2010; p xxxvii, 864 p.

5. Dyakonov, M. I., Spin physics in semiconductors. Springer: Berlin, 2008; p xviii, 439 p.

6. Yu, P. Y.; Cardona, M., Fundamentals of semiconductors : physics and materials

properties. 4th ed.; Springer: Berlin ; New York, 2010; p xx, 775 p.

7. Comprehensive semiconductor science and technology. Elsevier: Boston, MA, 2011.

8. Nirmal, M.; Brus, L., Luminescence photophysics in semiconductor nanocrystals. Accounts Chem Res 1999, 32, 407-414.

9. Burda, C.; Chen, X. B.; Narayanan, R.; El-Sayed, M. A., Chemistry and properties of nanocrystals of different shapes. Chem Rev 2005, 105, 1025-1102.

10. Salvador, M. R.; Hines, M. A.; Scholes, G. D., Exciton-bath coupling and inhomogeneous broadening in the optical spectroscopy of semiconductor quantum dots. $J$ Chem Phys 2003, 118, 9380-9388.

11. Cooney, R. R.; Sewall, S. L.; Dias, E. A.; Sagar, D. M.; Anderson, K. E. H.;

Kambhampati, P., Unified picture of electron and hole relaxation pathways in semiconductor quantum dots. Phys Rev B 2007, 75.

12. Lotfollahi, R. Electronic Structure of Surfaces 2006.

13. Mönch, W., Semiconductor surfaces and interfaces. 3rd, rev. ed.; Springer: Berlin ; New York, 2001; p xvi, 548 p.

14. Hill, N. A.; Whaley, K. B., A Theoretical-Study of the Influence of the Surface on the Electronic-Structure of Cdse Nanoclusters. J Chem Phys 1994, 100, 2831-2837.

15. Pokrant, S.; Whaley, K. B., Tight-binding studies of surface effects on electronic structure of CdSe nanocrystals: the role of organic ligands, surface reconstruction, and inorganic capping shells. Eur Phys J D 1999, 6, 255-267. 


\section{Chapter 3: Theory of vibrations and carrier transfer}

\subsection{Introduction}

The previous chapter described the electronic structure of semiconductor nanocrystals. This chapter presents the harmonic oscillator model which serves as the basis for the theoretical treatment of vibrations and carrier transfer in molecules and crystalline systems. First, the harmonic oscillator model is discussed and applied to atoms in a crystal lattice. Then, lightinduced transitions between quantum states are considered in both the time and frequency domains. This discussion is followed by a description of the Franck-Condon principle and its application to absorption and emission involving vibrational states. Finally, the classical and semiclassical versions of Marcus Theory are described using the harmonic oscillator framework.

\subsection{The Harmonic Oscillator}

Thus far, the approach utilized to explain the behaviour of atoms in crystals has considered their positions to remain fixed. A more realistic approach must incorporate the effects of vibrations that are present. These vibrations will be shown to play a key role in the electronic properties of these materials.

The classical approach to the study of vibrations involves imagining a mass attached to a spring in which displacement from some equilibrium length produce a restoring force $F$ which is directly proportional to the magnitude of the displacement: 


$$
F=m \frac{d^{2} x}{d t^{2}}=-k x
$$

This equation is called Hooke's Law, and the value $k$ is called the force constant of the spring, $m$ as the mass, $x$ is the displacement from equilibirum, and $t$ is time.

As force is the negative derivative of potential, $V$ :

$$
F=-\frac{d V}{d x}
$$

the potential of this system can be written as follows, assuming zero potential for the undistorted system:

$$
V(x)=\frac{1}{2} k x^{2}
$$

Solving the Schrödinger equation for a simple diatomic molecule using this potential where $\mathrm{x}$ represents internuclear distance, which is a good approximation of the real potential near the region of minimum energy, yields the following energy eigenvalues:

$$
E_{v}=\hbar \sqrt{\frac{k}{\mu}}\left(v+\frac{1}{2}\right)
$$

Here $\omega=\sqrt{\frac{k}{\mu}}$ is the fundamental frequency, $v$ is the vibrational quantum number, and $\mu$ is the reduced mass $\left(\mu=\frac{m_{1} m_{2}}{m_{1}+m_{2}}\right)$, where $m_{1}$ and $m_{2}$ are the masses of the two atoms. 
The wavefunctions which correspond to this system are depicted in Figure 3.1.

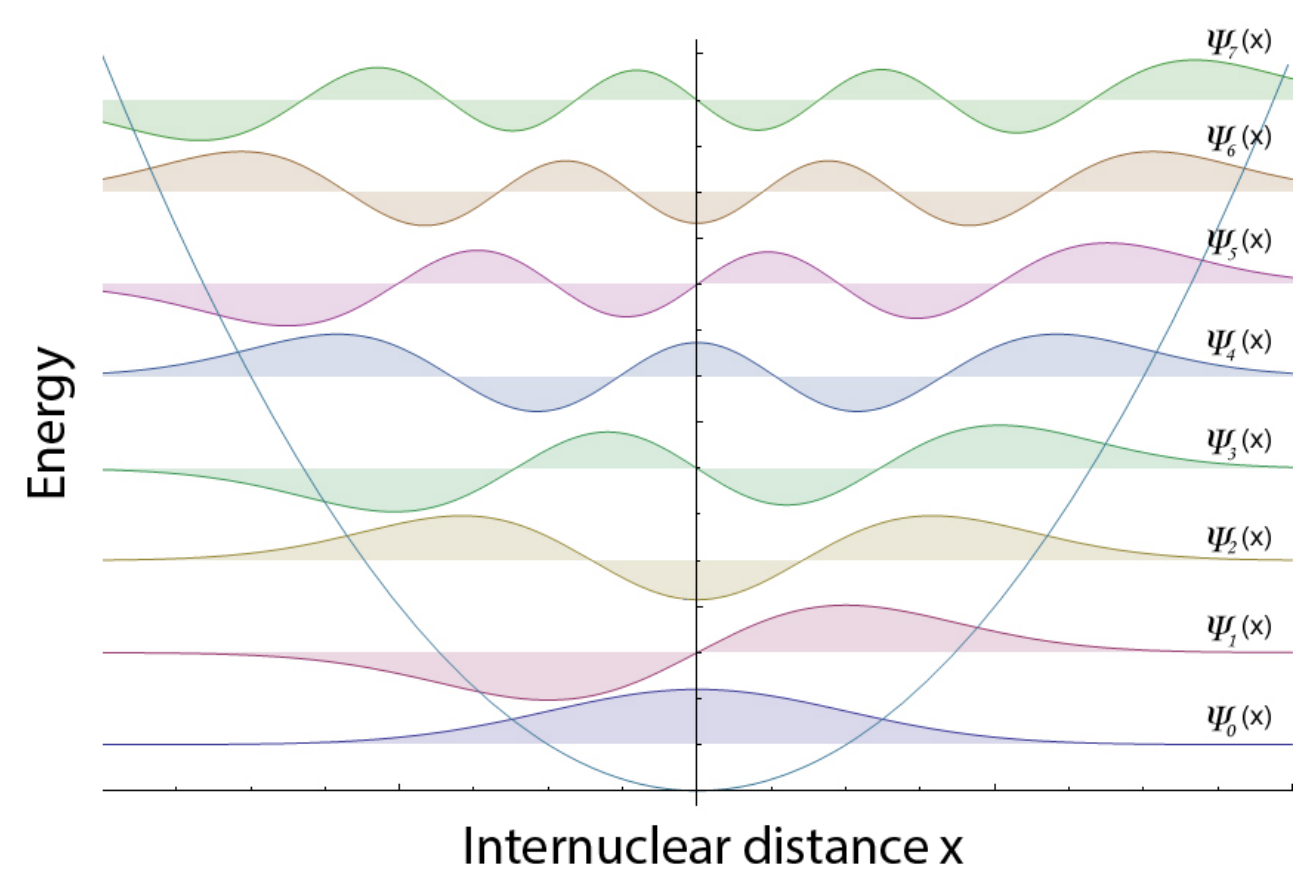

Figure 3.1 Harmonic oscillator wavefucntions superimposed on harmonic potential.

Adapted from work by AllenMcC. (File:HarmOsziFunktionen.jpg) [CC-BY-SA-3.0

(http://creativecommons.org/licenses/by-sa/3.0)], via Wikimedia Commons

Notably, some part of these wavefunctions "leaks" out of the classical potential barrier, a factor which will be revisited later in this chapter in the context of carrier transfer.

\subsection{Crystal vibrations}

Thus far the treatment of the harmonic oscillator has only been applied to simple diatomic systems. However, the same theory can be readily applied to atoms in a crystal lattice. Here, to preserve the very concept of a lattice, the "positions" of atoms within the lattice are 
assumed to be their equilibrium (as opposed to instantaneous) positions and the atoms are assumed be subject to a harmonic restoring force when displaced from these equilibrium positions (Hooke's law). ${ }^{1}$

One challenge encountered in crystalline systems is that changes in the positions of atoms are accompanied by changes in the distribution of electrons. However, by noting that the timescale of electronic motion is far shorter than that of nuclear motion, the adiabatic approximation can be invoked, in which it is assumed electrons react so quickly to lattice motions that at any moment they are in their ground state for a particular nuclear configuration. ${ }^{1}$

With these factors in mind, the system is constructed as a one-dimensional set of atoms separated by a fixed distance and subject to the potential described in Equation 3.3. In this equation, the coordinate refers only to the separation between nearest-neighbour atoms, such that the restoring force only acts on neighbouring atoms. As in the particle-in-a-box model from Chapter 1, the atoms at the end of the chain can be treated using the Born-von Karman boundary conditions. ${ }^{1}$ In such a model, all nearest neighbours are connected with springs of force constant $k$ and the unit cell may contain two atoms of different masses. Setting up Newton's equation for this system yields ${ }^{2}$ :

$$
\begin{aligned}
& M_{1} \ddot{u}_{n 1}+k\left(2 u_{n 1}-u_{n 2}-u_{n-1,2}\right)=0 \\
& M_{2} \ddot{u}_{n 2}+k\left(2 u_{n 2}-u_{n 1}-u_{n-1,1}\right)=0
\end{aligned}
$$

Here $M_{1}$ and $M_{2}$ refer to the masses of the atoms and the values $u_{n}$ represent the displacement of a given atom $n$ from its equilibrium position.

The above equations can be solved with an appropriate ansatz (Equation 3.7) 


$$
u_{n \alpha}=\frac{1}{\sqrt{M_{\alpha}}} u_{\alpha}(q) e^{i(q a n-\omega t)}
$$

Here $q$ is the phonon wavevector, $a$ is the lattice spacing, and $\omega$ is the frequency. Solving equations 3.5 and 3.6 by plugging in 3.7 yields the following relation between $\omega$ and $\mathrm{k}$ :

$$
\omega^{2}=k\left(\frac{1}{M_{1}}+\frac{1}{M_{2}}\right) \pm k\left[\left(\frac{1}{M_{1}}+\frac{1}{M_{2}}\right)^{2}-\frac{4}{M_{1} M_{2}} \sin ^{2} \frac{\mathrm{qa}}{2}\right]^{\frac{1}{2}}
$$

The result yields two branches of phonons with distinct dispersion curves, referred to as optical and acoustic (Figure 3.2). At $q=0$, the acoustic mode has $\omega=0$ while the optical mode has $\omega=\sqrt{\frac{2 f}{\mu}}$. At $q=\frac{\pi}{a}$, the acoustic mode has $\omega=\sqrt{\frac{2 f}{M_{1}}}$ while the acoustic mode has $\omega=\sqrt{\frac{2 f}{M_{2}}}$. In the acoustic branch, the ions within a unit cell move in phase, while in the optical branch, their motion is out of phase. $^{2}$ In three dimensions, there would be three acoustic and three optical modes whose energies would normally not be degenerate. More generally, in a threedimensional crystal, there are $3 n$ modes, where $n$ is the number of atoms per unit cell. These are divided into 3 acoustic modes and (3n-3) optical modes. 


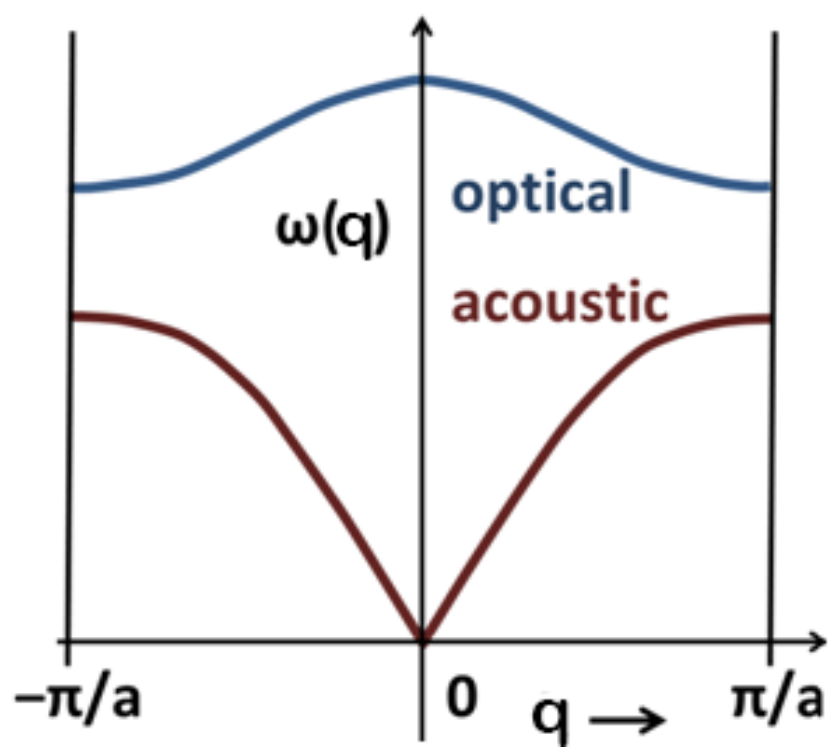

Figure 3.2. Solutions to the equations of motion for a linear diatomic chain of atoms. There are two branches, labeled acoustic and optical. By Brews ohare (Own work) [CC-BY-SA-3.0 (http://creativecommons.org/licenses/by-sa/3.0)], via Wikimedia Commons

Notably, the phonon energy can be shown to be formally identical to the energy of a collection of independent harmonic oscillators, where the collective displacement of atoms plays the role of " $x$ " in Equation 3.3 for each mode. ${ }^{3}$

\subsection{Transition probabilities in the time and frequency domains}

Before exploring the relationship between electronic and vibrational states, a brief overview of electronic transitions is in order. The probability of an electronic transition occurring between electronic states depends on the transition dipole moment:

$$
P=\frac{\pi}{2 \hbar^{2}}\left|\left\langle\psi^{\prime}\left|E_{0} \cdot \vec{\mu}\right| \psi\right\rangle\right|^{2}\left[\delta\left(\omega_{f i}-\omega\right)+\delta\left(\omega_{f i}+\omega\right)\right]
$$

Here $\omega_{f i}$ is the energy separation between the initial and final states, $\omega$ is the frequency of 
light, $\omega_{f i}$ is the difference in energy between final and initial states, $\psi$ is the wavefunction, $E_{0}$ is the magnitude of the electric field, and $\mu$ is the dipole operator.

Equation 3.9 is more commonly expressed as the net change in energy of the radiation field. To do so, a sum is carried out over all possible initial and final states, weighted by the probability of occupation of each state. Finally, a new value intensity $I(\omega)$, which is proportional to the net change in energy of the radiation, is defined, in which several terms in the equation are divided out in the definition to provide a simple expression:

$$
I(\omega)=\sum_{i, f} \rho_{i}|\langle f|\vec{\mu}| i\rangle|^{2}\left[\delta\left(\omega_{f i}-\omega\right)\right]
$$

Here $\rho_{i}$ is the probability of being found initially in state $i$,

Noting the wondrous properties of the Dirac delta function,

$$
\delta(\omega)=\frac{1}{2 \pi} \int_{-\infty}^{\infty} e^{i \omega t} d t
$$

this expression can be re-written as follows:

$$
I(\omega)=\frac{1}{2 \pi} \sum_{i, f} \rho_{i}\langle i|\vec{\mu}| f\rangle\langle f|\vec{\mu}| i\rangle \int_{-\infty}^{\infty} e^{i\left(\frac{E_{f}-E_{i}}{\hbar}-\omega\right) t} d t
$$

where $E_{f}$ and $E_{i}$ are the energies of the final and initial states, respectively.

The energy eigenvalues can be re-expressed using the Heisenberg operator ${ }^{4}$ 


$$
I(\omega)=\frac{1}{2 \pi} \int_{-\infty}^{\infty} \sum_{i, f} \rho_{i}\langle i|\vec{\mu}(0)| f\rangle\langle f|\vec{\mu}(t)| i\rangle e^{i \omega t} d t
$$

which incorporates the time-dependent Heisenberg Operator

$$
\mu(t)=e^{\frac{i H t}{\hbar}} \vec{\mu}(0) e^{\frac{-i H t}{\hbar}}
$$

where $H$ is the Hamiltonian of the matter.

Finally, invoking the completeness relation for states $f$ and using brackets to now represent an ensemble average over states $i$ yields ${ }^{5}$

$$
I(\omega)=\frac{1}{2 \pi} \int_{-\infty}^{\infty}\langle\vec{\mu}(0) \vec{\mu}(t)\rangle e^{i \omega t} d t
$$

This expression shows that the spectrum can be expressed as the Fourier transform of the dipole-dipole correlation function.

The time-correlation function in 3.15 reflects broadening due to homogeneous and inhomogenous factors:

$$
\langle\vec{\mu}(0) \vec{\mu}(t)\rangle \propto e^{i \omega_{f i} t-\frac{1}{2} \Theta^{2} t^{2}-\Gamma|t|} d t
$$

In this equation, $\Theta$ is an inhomogeneous broadening parameter, and $\Gamma$ is the homogeneous linewidth. Homogeneous broadening factors include lifetime and collisional broadening, while inhomogeneous broadening arises from the size distribution within the sample. These factors result in a spectrum containing physically-realistic peaks rather than delta-function transitions. 


\subsection{Franck-Condon principle}

Returning to vibrations in the molecular case, the vibrational energy surfaces described by equation 3.3 and the associated ladder of energies described by equation 3.5 will exist not only for the ground electronic state but also for any excited states as well. As pointed out by Franck in 1926 for molecules, these excited states may have potential energy curve minima at larger internuclear distance and smaller force constants owing to the weakening of bonds upon exictation. ${ }^{6}$ Huang and Rhys showed that this treatment could be extended to crystalline systems. $^{7}$

As above, the probability of a transition occurring between electronic states, each of which will possess a ladder of vibrational states, depends on the transition dipole moment. However, in this system, the Hamiltonian of the excited state will involve the potential of equation 3.3 with the replacement of $x$ with $(x-d)$, where $d$ is the shift in equilibrium internuclear distance relative to the ground state. 


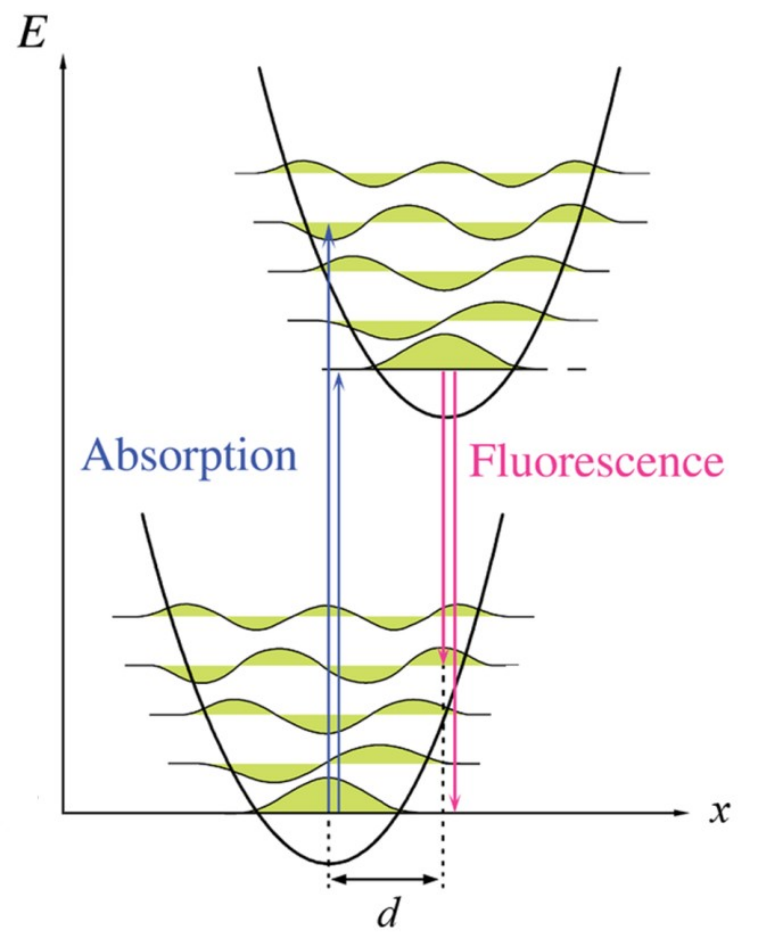

Figure 3.3. Illustration of the Franck-Condon principle in an energy diagram. Transitions probabilities depend on the overlap between the wavefunctions. By Mark M. Somoza (Own work) [GFDL (http://www.gnu.org/copyleft/fdl.html), CC-BY-SA-3.0

(http://creativecommons.org/licenses/by-sa/3.0/) or CC-BY-SA-2.5-2.0-1.0

(http://creativecommons.org/licenses/by-sa/2.5-2.0-1.0)], via Wikimedia Commons

By the Born-Oppenheimer approximation, this matrix element in Equation 3.9 can be partitioned into electronic $\left(\Psi_{e}\right)$ and vibration $\left(\Psi_{v}\right)$ components:

$$
\left\langle\psi^{\prime}\left|E_{0} \cdot \vec{\mu}\right| \psi\right\rangle=\left\langle\psi_{e}^{\prime}\left|E_{0} \cdot \vec{\mu}\right| \psi_{e}\right\rangle\left\langle\psi_{v}^{\prime} \mid \psi_{v}\right\rangle
$$


The vibrational component $\left\langle\psi_{v}^{\prime} \mid \psi_{v}\right\rangle$ is referred to as the Franck-Condon factor. Formally, the Franck-Condon factor must be determined by calculating the integral between the vibrational wavefunctions of the initial and final vibrational states. However, by making the simplifying assumption that the vibrational frequencies of the ground and excited states are equal and noting the relative displacement $d$ between the equilibrium internuclear distances of the two vibrational states, a simpler expression can be found. ${ }^{8}$ For absorption, the Franck-Condon factors are:

$$
\left|\left\langle 0 \mid v^{\prime}\right\rangle\right|^{2}=e^{-S} \frac{1}{v^{\prime} !} S^{v \prime}
$$

Here $v^{\prime}$ is the vibrational quantum number from the harmonic oscillator model and $\mathrm{S}$ is called the Huang-Rhys parameter and is related to the relative displacement $d$ by $S=\frac{d^{2} \mu \omega_{0}}{2 \hbar}$.

Thus, the absorption spectrum can be written as:

$$
\sigma_{a b s}(\omega)=\left|\mu_{e g}\right|^{2} \sum_{v^{\prime}=0}^{\infty} e^{-S} \frac{1}{v^{\prime} !} S^{v \prime} \delta\left(\omega-\omega_{e g}-v^{\prime} \omega_{0}\right)
$$

Here $\mu_{e g}$ is the overlap between electronic wavefunctions, $\omega_{0}$ is the vibrational frequency, and $\omega_{e g}$ is the electronic energy difference between states. Again, the delta function ensures conservation of energy between the absorbed photon and the energy difference between the initial and final states.

The transition probabilities depend on the degree of overlap between the vibrational wavefunctions on the two potential energy surfaces for a given nuclear configuration, which is 
dependent upon the displacement $d$ and thus $\mathrm{S}$ (Figure 3.3). When $\mathrm{S}=0$, there is no difference between the energy minima of the ground and excited states. As a result, the transition occurs completely between the $v=0$ to $v^{\prime}=0$ vibrational states (Figure 3.4), so the only Franck-Condon factor with any weight involves $v^{\prime}=0$. For a displacement of $0<\mathrm{S}<1$, there is a small probability of transition to some of the lower vibrational states above $v^{\prime}=0$ (Figure 3.4). Larger values of $\mathrm{S}>1$ show a Poisson distribution of transition probabilities (Figure 3.4) with a maximum for absorption into a higher $v^{\prime}$ state. Notably, the value of S can be easily correlated to the ratio between the $v^{\prime}=0$ and $v^{\prime}=1$ peaks in the spectrum.

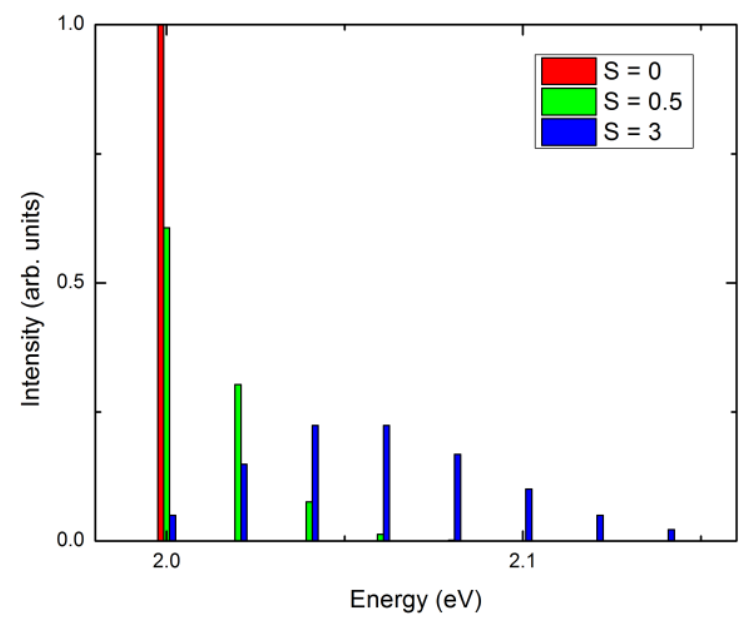

Figure 3.4. Effect of displacement, $S$, on the transition probability between vibrational states. Small values of S result in transitions mostly or exclusively involving the $0-0$ vibrational states, while larger values lead to a Poisson distribution among the final vibrational states.

The envelope of these transitions will have a Gaussian profile arising from equation 3.17 with a maximum at ${ }^{9}$ 


$$
\omega=\omega_{e g}+S \omega_{0}
$$

In addition, the excess vibrational energy present in the final electronic state upon excitation at the initial electronic state potential minimum can be defined as:

$$
\lambda=S \hbar \omega_{0}
$$

This term, $\lambda$, is called the reorganization energy. Notably, since vibrational energy is quickly dissipated on the femtosecond to picoseconds timescale and emission occurs from the lowest vibrational state of the excited electronic state, the fluorescence spectrum will be shifted by $2 \lambda$ from the absorption spectrum and mirror it (Figure 3.5). In reality, the transitions do not appear as delta functions in the experimental spectrum due to the broadening factors discussed in equation 3.16. 


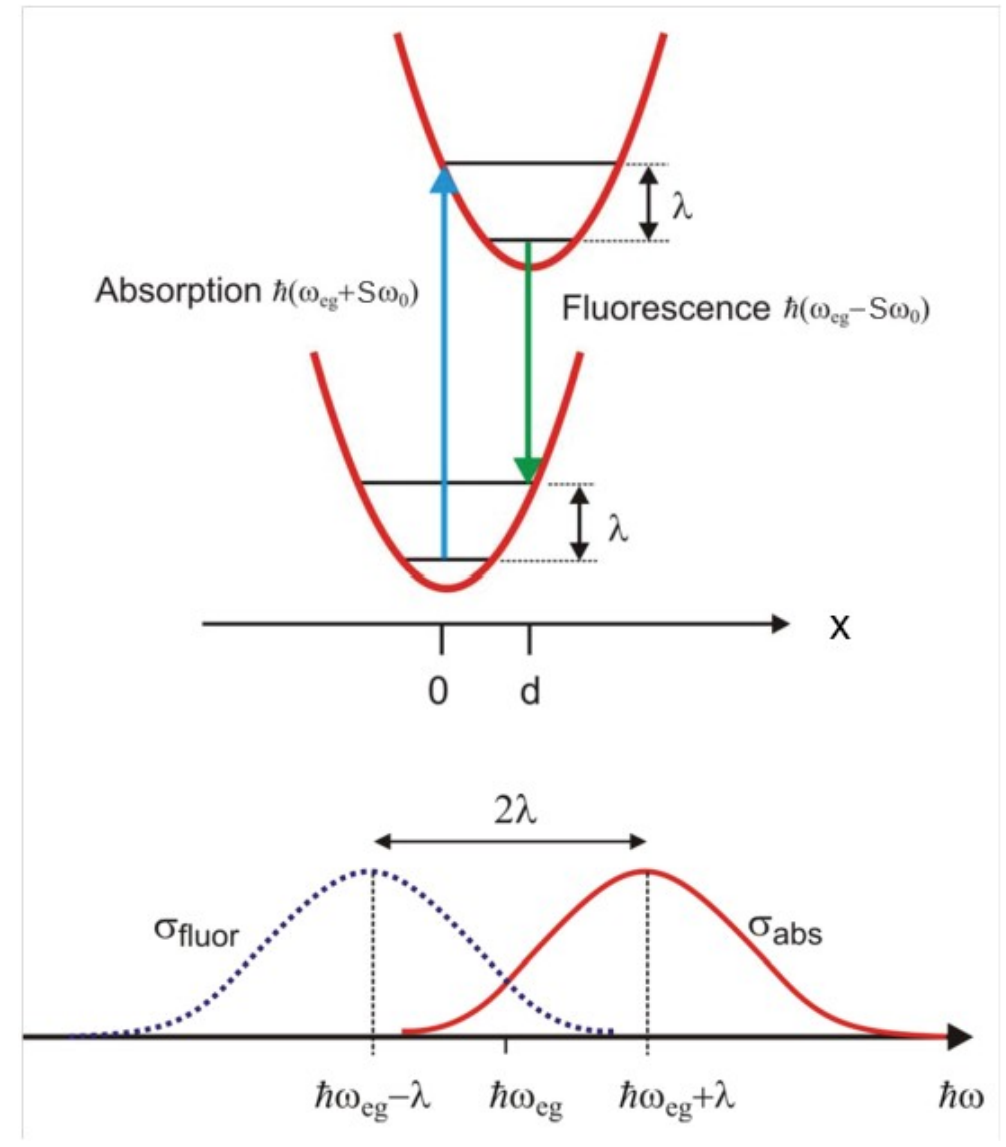

Figure 3.5. Illustration of the offset between the absorption and emission spectra by a factor of

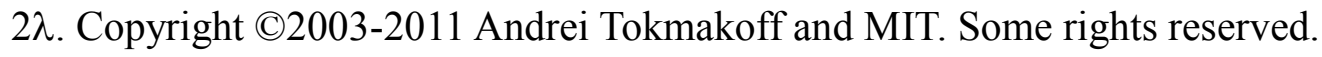
http://ocw.mit.edu/terms/

\subsection{Classical Marcus theory of electron transfer}

The above description of the harmonic oscillator provides a good foundation for discussing Marcus theory of electron transfer. In the case of electron transfer, nonradiative transitions take place due to the intersection of two vibrational potential curves rather than due to vertical transitions of absorption or emission between electronic states. Consider a pair of 
intersecting parabolic potential curves with minima at $x_{r}$ and $x_{p}$ and separated by energy $\Delta G^{0}$ (Figure 3.6). Here the ordinate is Gibbs free energy, employed to take account of changes in density of states as well as energy. The abscissa is a coordinate accounting for the polarization and vibrational difference between the two states. The potential surfaces are assumed to be harmonic in nature and to possess the same force constant. In this formulation, the barrier occurs at

$$
V\left(x_{r}\right)=V\left(x_{p}\right)
$$

where $V\left(x_{a}\right)$ is the potential at the value $a$ of coordinate $x$.

$$
\begin{gathered}
\text { or } \\
\frac{1}{2} k\left(x_{c}-x_{r}\right)^{2}=\Delta G^{0}+\frac{1}{2} k\left(x_{c}-x_{p}\right)^{2}
\end{gathered}
$$

where the potential has been replaced by the definition of potential from equation 3.3, accounting for the free energy difference $\Delta G^{0}$.

Solving for $x_{c}$ yields:

$$
x_{c}=\frac{\Delta G^{0}}{k\left(x_{p}-x_{r}\right)}+\frac{\left(x_{p}+x_{r}\right)}{2}
$$




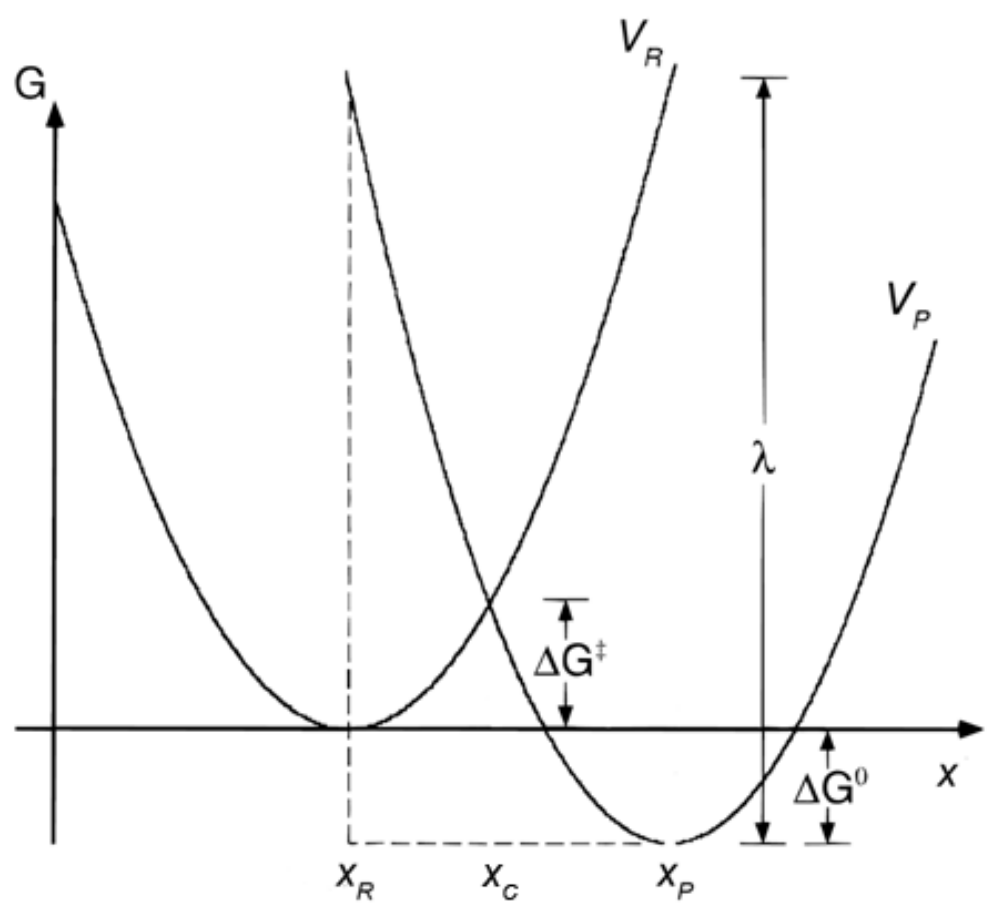

Figure 3.6. Schematic of displaced harmonic oscillators to explore electron transfer, indicating values of reorganization energy, $\lambda$, free energy difference $\Delta \mathrm{G}^{0}$, and free energy barrier $\Delta \mathrm{G}^{\ddagger}$. Reprinted with permission from "Contemporary Issues in Electron Transfer Research" Paul F. Barbara, Thomas J. Meyer, and Mark A. Ratner The Journal of Physical Chemistry 1996100 (31), 13148-13168). Copyright 1996 American Chemical Society.

The reorganization energy $\lambda$ may also be defined as the potential difference between the equilibrium positions of the two curves:

$$
\lambda=\frac{1}{2} k\left(x_{r}-x_{p}\right)^{2}
$$


The free energy barrier $\Delta \mathrm{G}^{\ddagger}$ is equal to

$$
\Delta G^{\ddagger}=\frac{1}{2} k\left(x_{c}-x_{r}\right)^{2}
$$

Utilizing equations 3.24 and 3.26, the free energy barrier becomes

$$
\Delta G^{\ddagger}=\frac{\left(\Delta G^{0}+\lambda\right)^{2}}{4 \lambda}
$$

Thus the activation energy can be defined in terms two fundamental quantities: the free energy difference $\Delta \mathrm{G}^{0}$ between the two states and the reorganization energy $\lambda$, which is a function of the separation between the two states.

By analogy with the Arrhenius equality and the Eyring equation from transition state theory, Marcus derived the following expression for the rate of electron transfer:

$$
\mathrm{k}_{\mathrm{ET}}=\mathrm{Ae}^{\frac{-\left(\Delta G^{0}+\lambda\right)^{2}}{4 \lambda k_{B} T}}
$$

Here $A$ is a prefactor that depends on the frequency of crossing the barrier top and depends on the degree of electronic coupling between the initial and final states. Values of $A$ vary widely depending on the particular system, resulting in electron transfer rates ranging from the femtosecond timescale to almost inifinitely slow.

Notably, as Marcus Theory was being developed as a means to explain electron transfer in molecules, advances in the solid state physics community led to the development of an equivalent theory to explain carrier transfer in crystals. Huang and Rhys first developed this 
theory of nonradiative transitions, a process referred to in the physics literature as multiphonon emission (MPE). In their original work, this kind of transition was restricted to interactions with longitudinal optical (LO) phonons of fixed frequency. ${ }^{7}$ Following advances by Kovarskii and Sinyavskii, in the 1970s, Lang and Henry presented a compelling model of recombination from deep levels of semiconductors using a configuration coordinate approach. ${ }^{10,11}$

\subsection{Marcus-Jortner electron transfer theory}

One of the major limitations of classical Marcus Theory as described above lies in its predictive power for electron transfer rates at low temperature. Classical Marcus Theory predicts a vanishing rate constant as temperature approaches zero. But experimental data on the bacterium Chromatium shows that the rate becomes temperature-independent but nonzero in the low-temperature regime. ${ }^{12,13}$

Marcus theory can be improved by incorporating the possibility of quantum-mechanical tunneling through the activation barrier. In classical Marcus theory, only low-frequency modes corresponding to the polarization changes in external medium are considered to affect the rate of electron transfer, a treatment that is typically reasonable at high-temperature. However, highfrequency modes corresponding to vibrations within the charge transfer system itself also exist. In Marcus-Jortner theory, both low-frequency modes of the medium and high-frequency "intramolecular" or "inner sphere" modes of the system in which tunneling may be significant are considered. ${ }^{13}$ For simplicity, these high-frequency "quantum" modes are characterized by a mean frequency $\omega$ and an effective electron-phonon coupling strength, $S$, while the low 
frequency modes are similarly specified by a single mean frequency and an effective coupling strength together represented by the reorganization energy $\lambda_{m} \cdot{ }^{13}$ Jortner refers to this simplification an "Einstein-type" approximation. ${ }^{13}$

Under the conditions of thermal excitation of the medium phonon modes but insufficient thermal energy to excite the quantum modes (semiclassical Marcus Theory), Jortner derives the following expression for the rate of electron-transfer ${ }^{13}$ :

$$
k_{E T}=\frac{2 \pi}{\hbar} H_{R P}^{2}\left(\frac{\pi}{\hbar^{2} \lambda_{m} k_{b} T}\right)^{\frac{1}{2}} \sum_{v^{\prime}} e^{-S} \frac{S^{v^{\prime}}}{v^{\prime} !} e^{\frac{-\left(\Delta G^{0}+\lambda_{m}+v^{\prime} \hbar \omega\right)^{2}}{4 \lambda_{m} k_{b} T}}
$$

In this expression, $\mathrm{H}_{\mathrm{RP}}$ is the electronic matrix element of the reactant and product states, $\lambda_{\mathrm{m}}$ is the reorganization energy of the low frequency mode, $S$ is the coupling strength of the high frequency mode and $\omega$ is its frequency, and $v^{\prime}$ is the index of excited vibrational states.

While this expression appears complicated at first, it is relatively easily understood. The expression emerges from the golden rule in perturbation theory. $\mathrm{H}_{\mathrm{RP}}$ is the electronic matrix element for the reactant and product states. The terms prior to the summation include the classical density of states. ${ }^{14}$ The first term in the summation is the sum of all possible FrankCondon vibrational overlaps. The exponential part of the term represents the populations having the energy required to undergo electron transfer with energy conservation. The terms that dominate the sum are those for which $\left|\Delta \mathrm{G}^{0}\right| \approx \lambda_{\mathrm{m}}+v^{\prime} \hbar \omega$, where $\Delta \mathrm{G}^{0}$ is a negative quantity representing the energy released. Performing the summation over product vibrational states is what permits tunneling, in contrast to classical Marcus theory. Marcus-Jortner theory has been 
shown to better account for electron transfer rates at low temperature and to address other shortcomings of classical Marcus theory. ${ }^{14}$

\section{References}

1. Ashcroft, N. W.; Mermin, N. D., Solid state physics. Holt: New York,, 1976; p xxi, 826 p. 2. Ibach, H.; Lüth, H., Solid-state physics : an introduction to principles of materials science. 4th extensively updated and enlarged ed.; Springer: Berlin ; New York, 2009; p xiv, 533

p. 3. Kaxiras, E., Atomic and electronic structure of solids. Cambridge University Press: Cambridge, UK ; New York, 2003; p xx, 676 p.

4. Gordon, R. G., Correlation Functions for Molecular Motion. In Advances in magnetic and optical resonance, Waugh, J. S., Ed. Academic Press: San Diego, 1990; pp 1-42.

5. Tokmakoff, A., Time Correlation Functions. In Time Dependent Quantum Mechanics and Spectroscopy, MIT OpenCourseWare: 2011.

6. Franck, J., Elementary processes of photochemical reactions. T Faraday Soc 1926, 21, 0536-0542.

7. Huang, K.; Rhys, A., Theory of Light Absorption and Non-Radiative Transitions in FCentres. Proc R Soc Lon Ser-A 1950, 204, 406-423.

8. Kelley, A. M., Electronic Spectroscopy. In Condensed-Phase Molecular Spectroscopy and Photophysics, John Wiley \& Sons, Inc.: 2012; pp 139-162.

9. Tokmakoff, A., Electronic Spectroscopy: Displaced Harmonic Oscillator Model. In TimeDependent Quantum Mechanics and Spectroscopy, Massachusetts Institute of Technology: 2009. 10. Lang, D. V.; Henry, C. H., Nonradiative Recombination at Deep Levels in Gaas and Gap by Lattice-Relaxation Multiphonon Emission. Phys Rev Lett 1975, 35, 1525-1528.

11. Henry, C. H.; Lang, D. V., Nonradiative Capture and Recombination by Multiphonon Emission in Gaas and Gap. Phys Rev B 1977, 15, 989-1016.

12. Hopfield, J. J., Electron-Transfer between Biological Molecules by Thermally Activated Tunneling. P Natl Acad Sci USA 1974, 71, 3640-3644.

13. Jortner, J., Temperature-Dependent Activation-Energy for Electron-Transfer between Biological Molecules. J Chem Phys 1976, 64, 4860-4867.

14. Barbara, P. F.; Meyer, T. J.; Ratner, M. A., Contemporary issues in electron transfer research. J Phys Chem-Us 1996, 100, 13148-13168. 


\section{Chapter 4: Surface trapping in semiconductor nanocrystals}

*This chapter was partially adapted from Mooney, Jonathan, et al. "Challenge to the deep-trap model of the surface in semiconductor nanocrystals." Physical Review B 87.8 (2013): 081201. and Mooney, Jonathan, et al. "A microscopic picture of surface charge trapping in semiconductor nanocrystals." The Journal of chemical physics 138.20 (2013): 204705.

\subsection{Introduction}

With the theoretical foundation offered in prior chapters, it is possible to explore carrier trapping and emission in semiconductor nanocrystals (NCs). This chapter examines experimental data obtained over a broad temperature range of emission from semiconductor NCs. These data are analyzed in the framework of classical (Marcus) and semiclassical (MarcusJortner) electron transfer (ET) theory. Semiclassical ET theory is found to properly account for both the observed temperature-dependence as well as the spectral properties of surface emission. A model for understanding surface trapping in terms of a strongly coupled surface state is discussed.

\subsection{Temperature-dependent emission from semiconductor nanocrystals}

Steady-state photoluminescence (PL) spectra over a broad temperature range were obtained on samples of CdSe, CdS, and CdSe/ZnS NCs with radii ranging from $1.15 \mathrm{~nm}$ to 2.04 $\mathrm{nm}$, with size distributions within the sample of $5-10 \%$ for $\mathrm{CdSe}$ and $\sim 20 \%$ for $\mathrm{CdS}$. 
Experimental details are noted in Appendix A. Fig. 4.1(a) shows PL spectra of a characteristic sample of CdSe NCs, with the band edge absorption at $2.50 \mathrm{eV}$ and a corresponding nanocrystal radius of $1.15 \mathrm{~nm}$ determined from the energy of the band edge absorption feature. ${ }^{1}$ The electronic structure of CdSe NCs is well-known, and emission arises from the lowest-energy "band edge" quantized core excitonic state ${ }^{2}$. This PL peak is visible near $2.44 \mathrm{eV}$ in Fig. 4.1(a). The core PL blueshifts and narrows as temperature decreases due to lattice contraction (see R dependence in Equation 2.7) and electron-phonon interactions, consistent with prior work. ${ }^{3}$ Here, an excitation energy resonant with the band-edge exciton (1S peak) is employed at all temperatures to ensure consistency between measurements by minimizing trapping of hot (excess energy) excitons to surface states. ${ }^{4-8}$

As expected for a surface-passivated $\mathrm{NC},{ }^{9,10}$ at room temperature no low-energy trap emission is visible from CdSe [Fig. 4.1(a)]. Our samples exhibited $\sim 10 \%$ quantum yield at 300 $\mathrm{K}$, consistent with prior works. ${ }^{2,11,12}$ As temperature is decreased, however, low-energy surface emission presents itself in the spectrum, becoming prominent ( $>10 \%$ of total emission) below $200 \mathrm{~K}$. Similar observations have been reported previously. ${ }^{13,14}$ Notably, as temperature is lowered below $70 \mathrm{~K}$, the surface PL intensity consistently decreases.

Fig. 4.1(b) shows PL spectra of CdS NCs $(\mathrm{R}=2.04 \mathrm{~nm})$. While the general trends are the same as for CdSe, there are two major differences: in CdS NCs surface emission is present at room temperature and it is similar in intensity to core emission. This observation has historically been rationalized by suggesting a lower surface quality of $\mathrm{CdS}$ creating more surface traps from which the PL arises. ${ }^{15}$ 

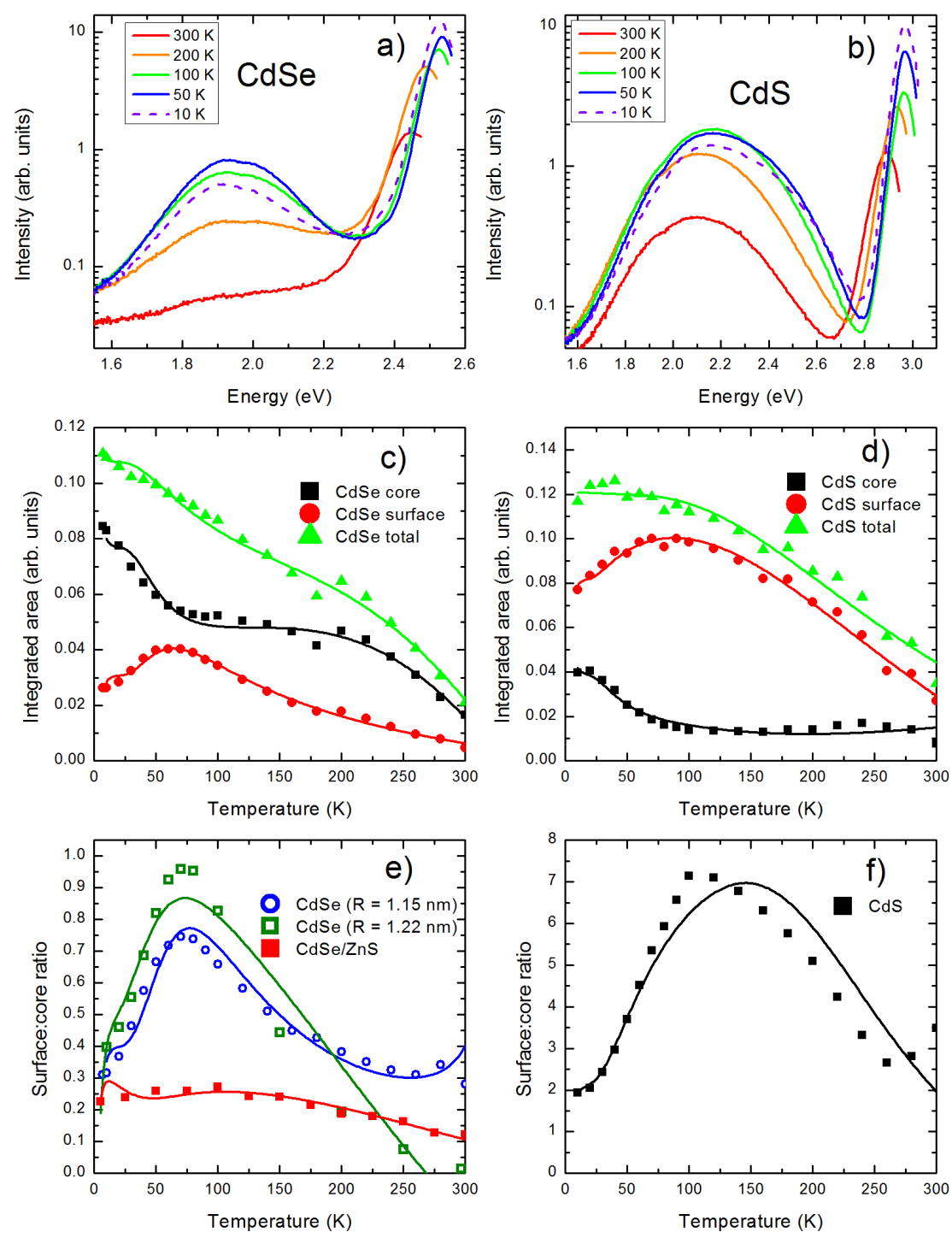

Figure 4.1. PL spectra of $\mathrm{CdSe}(\mathrm{R}=1.15 \mathrm{~nm})$ (a) and $\mathrm{CdS}$ (b) $\mathrm{NC}$ from $10 \mathrm{~K}$ to $300 \mathrm{~K}$. The core PL is at higher energies while the surface PL is broader and redshifted. Integrated areas of the core and surface lineshape bands for CdSe $(\mathrm{R}=1.15 \mathrm{~nm})(\mathrm{c})$, and $\mathrm{CdS}(\mathrm{d})$, show the turnover points in surface emission. Temperature dependence of the relative lineshape areas for two sizes of $\mathrm{CdSe}(\mathrm{e}), \mathrm{CdSe} / \mathrm{ZnS}$ and $\mathrm{CdS}$, (f). In Fig. 4.1c)-f), symbols represent experimental data, while solid lines represent the fits described in Section 4.6. 


\subsection{Analysis of emission trends}

To quantitatively analyze the results, the spectra presented in energy units were converted into lineshapes for further analysis by factoring out the $v^{3}$ (frequency cubed) dependence of spontaneous emission. ${ }^{16}$ The band edge and surface bands in the lineshapes were then simultaneously fitted to a Voigt peak and a Gaussian peak, respectively. The areas of these peaks provide a more meaningful measure of the exciton populations of the two states. ${ }^{16}$

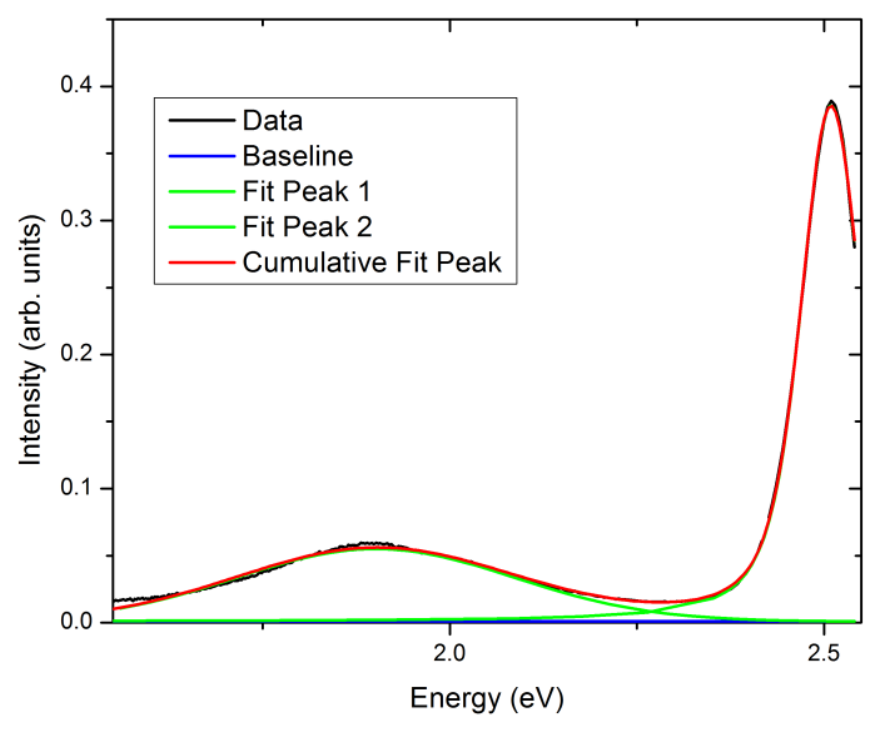

Figure 4.2. PL spectra of CdSe NC linehspes simultaneously fitted to a Voight peak (band edge peak) and a Gaussian peak (surface peak) to determine lineshape areas.

Fig. 4.1(c) (4.1(d)) shows the lineshape peak area (integrated intensity) of the core, surface, and total emission for CdSe (CdS). Also shown are the relative areas of the two bands 
[Figs. 4.1(e) and 4.1(f), points]. For CdSe (Fig 4.1c), the core PL increases at low temperature precisely as expected. ${ }^{3}$ The surface PL increases, albeit with a stronger temperature dependence. What is newly apparent from the lineshape area analysis is that the decrease in surface lineshape area from $70 \mathrm{~K}$ to $10 \mathrm{~K}$ complements a corresponding increase in core lineshape area. This complementarity suggests that thermodynamic population exchange can take place between the two states and that the populations of the two states are in some way correlated.

In CdS NCs (Fig. 4.1d), surface lineshape area increases as temperature drops down to $\sim 100 \mathrm{~K}$, while core lineshape area shows little temperature-dependence over this range. However, as temperature drops below $100 \mathrm{~K}$, surface lineshape area decreases while core lineshape area grows substantially.

The total lineshape areas (core + surface) for the various NCs studied are also presented in Fig. 4.1(c and d). As temperature is decreased, there is a rather consistent increase in total lineshape area over the entire temperature range studied. That is, even as intensity shifts from the surface lineshape band to the core lineshape band below an intermediate temperature, as discussed above, the cumulative lineshape area does not decrease as temperature is lowered. Such an observation again suggests population exchange between the two states.

An analysis of another size of CdSe NCs ( $\mathrm{R}=1.22 \mathrm{~nm}$, Fig. 4.1e) and CdSe/ZnS (Fig 4.1e) shows the same basic trends. The ratios presented in Figures $4.1 \mathrm{e}$ and $4.1 \mathrm{f}$ provide a very clear demonstration that the relative amount of surface lineshape area is a highly temperaturedependent property and reaches a distinct maximum at an intermediate temperature between 10 $\mathrm{K}$ and $300 \mathrm{~K}$, typically in the range $70 \mathrm{~K}-100 \mathrm{~K}$, for all samples. What is notable is that regardless of whether, at intermediate temperatures, the core lineshape areas are similar in 
magnitude to the surface $(\mathrm{R}=1.22 \mathrm{~nm}$, Fig. $4.1 \mathrm{e})$ or significantly greater than the surface $(\mathrm{R}=$ $1.15 \mathrm{~nm}$, Fig. 4.1e.), this behaviour is identical, a finding our group has recently extended to ultrasmall CdSe NCs in which core lineshape area is significantly less than that of the surface at room temperature. ${ }^{17}$ In other words, CdSe NCs display the same temperature-dependent behaviour regardless of the relative amounts of surface:core lineshape area.

\subsection{Theoretical approaches to understanding surface trapping}

While these data are consistent with prior experimental works, they are remarkably inconsistent with existing descriptions of the nature of the NC states which give rise to the surface PL. The standard model for describing the origin of the surface PL invokes an energetically broad distribution of surface localized defect states. ${ }^{11,12}$ These surface defects are expected to lie deep within the bandgap, thereby rationalizing the $300-500 \mathrm{meV}$ redshift $(\delta \mathrm{E})$ of the surface PL with respect to the band edge core excitonic PL. Similarly, the $300-500 \mathrm{meV}$ bandwidth $(\Delta \mathrm{E})$ of the surface PL is rationalized by a broad energetic distribution of these surface defects. Under these conditions, this standard model suggests highly exergonic, irreversible relaxation can occur from the 1S core excitonic state to these surface defect states, which can then emit light with negligible temperature dependence relative to the core [Fig. 4.2(a) and 4.2(b)] since the redshift is an order of magnitude larger than available thermal energies, $\delta \mathrm{E}$

$>10 \mathrm{k}_{\mathrm{B}} \mathrm{T} .{ }^{11,18}$ Such a model rationalizes the breadth and redshifting of the surface PL, but completely fails to account for the temperature dependence [Fig. 4.1(c) and 4.1(d)]. Indeed, such a model would predict no expected temperature dependence of integrated surface PL relative to core PL, in stark contrast to the data. 
The rich temperature dependence of the relative areas immediately suggests a thermally activated process by which population exchange can occur between the core excitonic states and the surface states. This view requires a configuration coordinate approach in order to introduce energetic barriers which dictate the thermodynamics of core and surface populations. Classical Marcus electron transfer (ET) theory has been recently used by Jones and Scholes ${ }^{9}$ to explain the temperature dependence of PL lifetimes. This approach treats carrier trapping as a classical ET reaction in which a delocalized exciton state is converted into a state in which a charge carrier is largely localized on the surface. Notably, the configuration coordinate approach of Marcus ET theory is equivalent to independently-developed theories from solid-state physics for deep traps in semiconductors and has been used by Brus to explain carrier trapping on surface states of $\mathrm{Si}$ nanocrystals. ${ }^{19}$

This configuration coordinate approach based upon classical Marcus electron transfer (ET) theory can rationalize the temperature dependence of the PL ratio, Fig. 4.2(c) and 4.2(d). Specifically, a configuration involving a surface state lower in energy $\left(\Delta \mathrm{G}^{0}\right)$ but within $\mathrm{k}_{\mathrm{B}} \mathrm{T}$ of the core state and with a core-to-surface activation barrier $\left(\Delta \mathrm{G}^{\ddagger}\right)$ smaller than $\Delta \mathrm{G}^{0}$ can correctly account for the observed temperature dependence of the PL ratios. As temperature is lowered, the decrease in thermal energy will preferentially populate the surface state in a Boltzmann-like manner. However, below some temperature, population will be unable to classically surmount $\Delta \mathrm{G}^{\ddagger}$, leading to an increase in core state population. Such a trend would give rise to the observed peak in the surface:core ratio. 

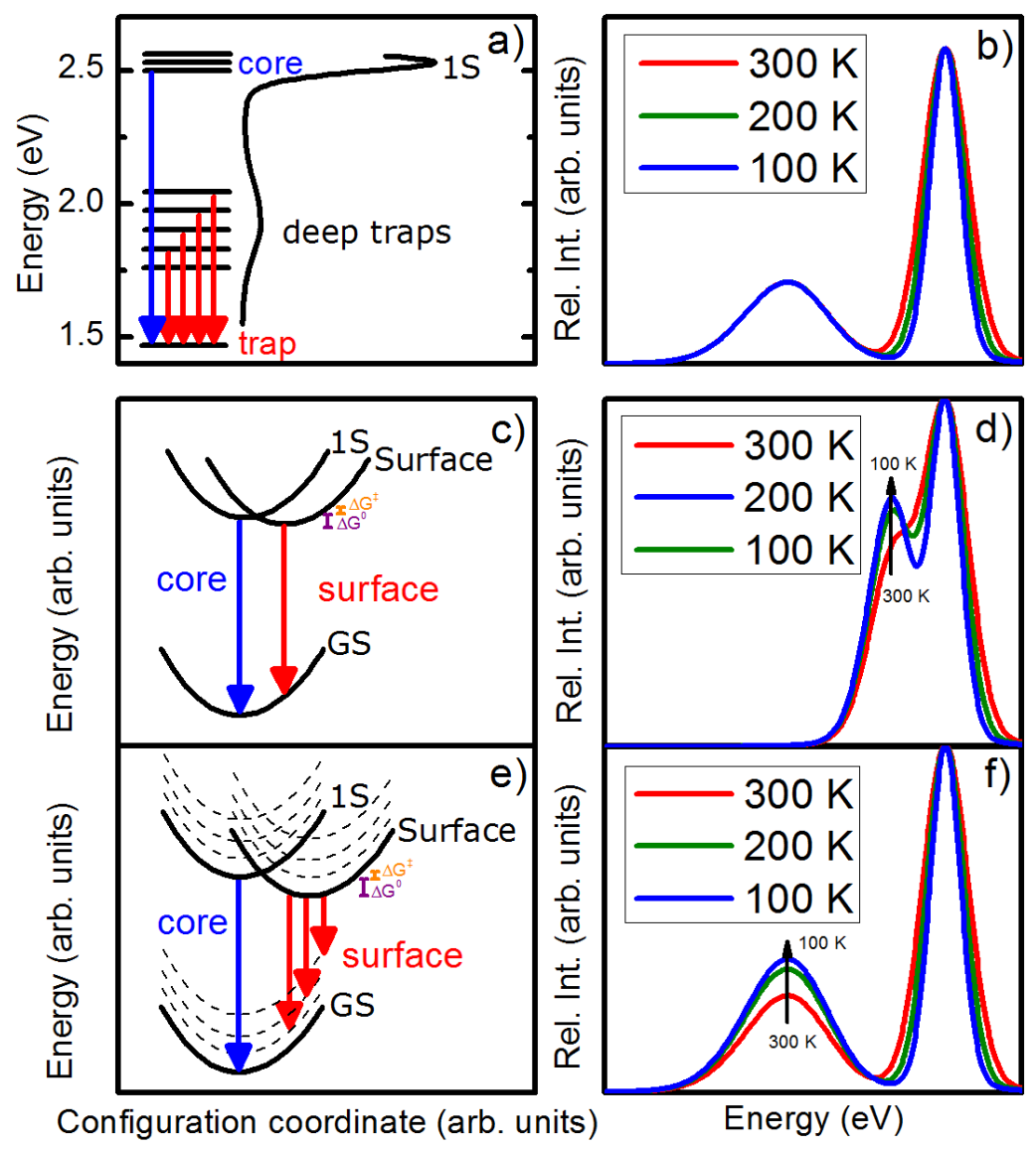

Figure 4.3. Three models for core and surface emission from NCs. The standard energy level picture of surface PL invokes a distribution of deep traps shown in the exciton representation, (a). Due the depth of these traps, the relative PL spectra would exhibit no temperature dependence, (b). A configuration coordinate picture using classical electron transfer (ET) theory to describe thermally activated population transfer from the core to the surface, (c). The simulated spectra would show temperature dependence, but with little broadening and redshifting, (d). A minimal configuration coordinate picture using a semiclassical ET theory, (e). The solid line represents the classical mode and the dashed lines represent the quantum mode, with displacements implicit. The small free energy difference along the classical bath coordinate gives rise to the temperature dependence of the surface PL, whereas the large coupling to the quantum (phonon) modes gives rise to the broadening and redshifting, (f) 


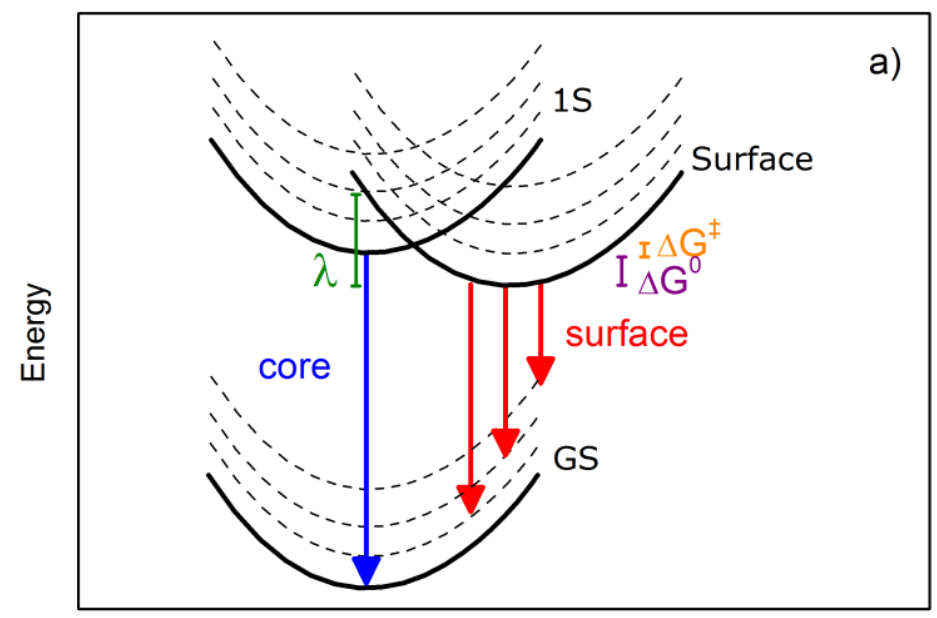

Configuration coordinate (arb. units)

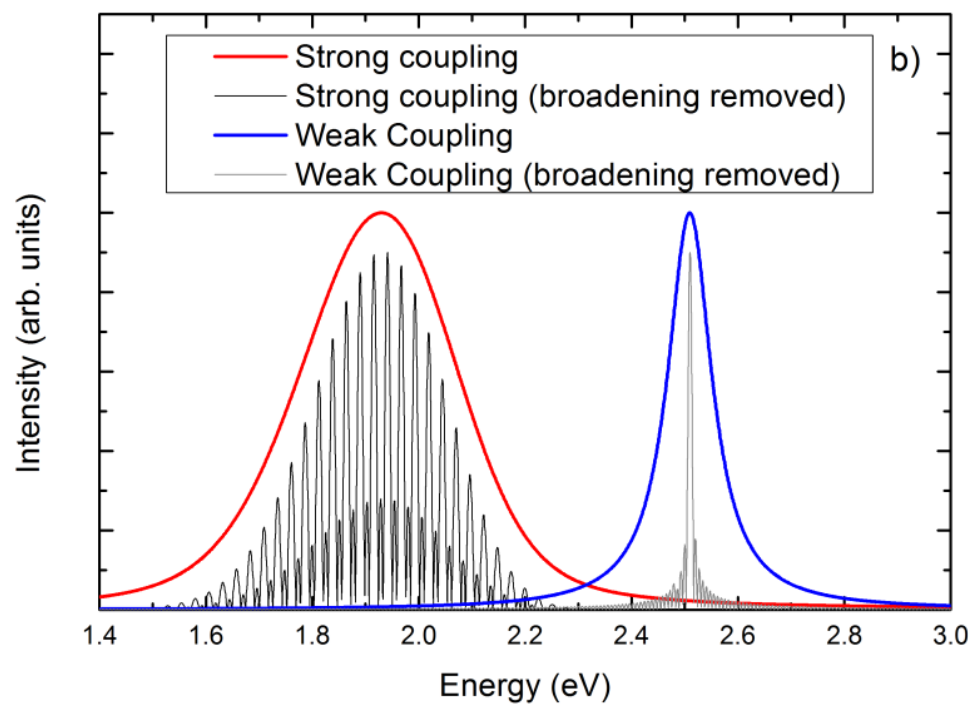

Figure 4.4. Schematic representation of electron-transfer. A displaced (strongly-coupled) harmonic oscillator will emit into several vibrational levels of the ground-state, while an undisplaced oscillator will emit primarily into one state (a). With stronger coupling, a broader and redshifted spectrum is observed (b). The effect of incorporating broadening factors (Equation 3.16) is illustrated. 
To demonstrate how Marcus theory with the appropriate parameters can explain the observed data, the rates for forward and back electron transfer were calculated according to traditional Marcus theory (Equation 3.35) using a model system of three states (Fig 4.3) in which electron transfer occurs with the parameters $\Delta \mathrm{G}^{0}=10 \mathrm{meV}$ and $\lambda=300 \mathrm{meV}$. These rates are plotted as a function of temperature in Fig. 4.5. The back rate is always slower than the forward rate owing to the larger value of $\Delta \mathrm{G}^{\ddagger}$ for the back reaction. Moreover, because $\Delta \mathrm{G}^{\ddagger}$ is greater for the back reaction, the relative difference in the two rates (i.e. the ratio between rates) grows as temperature decreases due to the exponential dependence of the rate on $-\Delta G^{\ddagger} / k_{B} T$ (Fig 4.5).

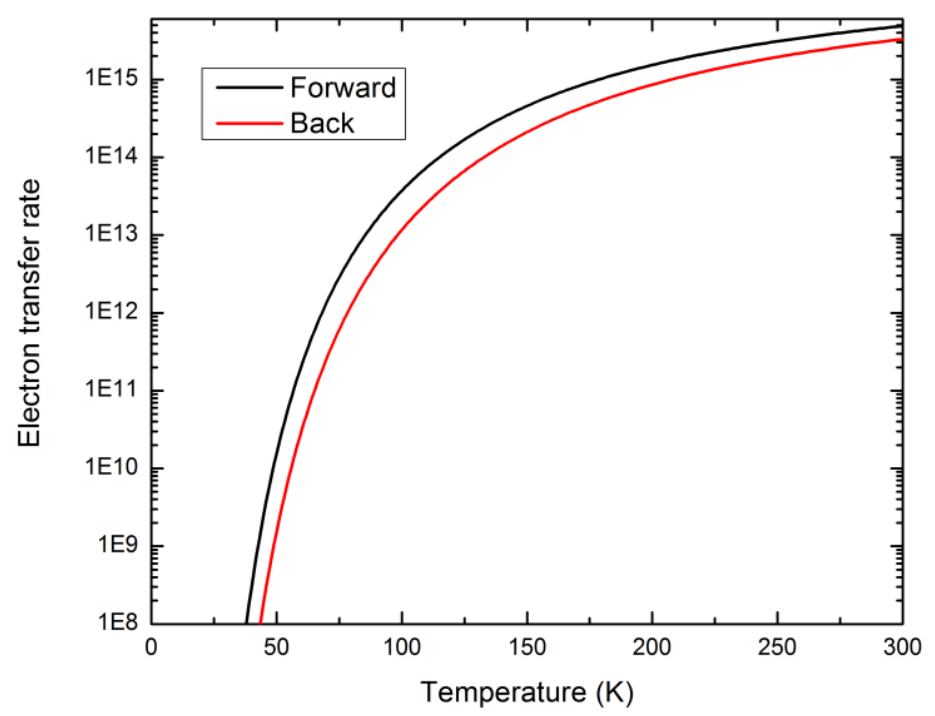

Figure 4.5. Calculated rates of forward and back electron transfer show a back transfer rate that is smaller than the forward rate, a difference that grows as temperature is lowered.

The more rapid falling off of the back electron transfer rate relative to the forward rate with decreasing temperature predicts that the relative population of the surface state will increase as temperature drops, resulting a Boltzmann-like trend for relative population with temperature. 
Below a given temperature, however, the activation barrier $\Delta \mathrm{G}^{\ddagger}$ to forward trapping becomes significant relative to thermal energy and the trapping rate slows to less than that of the emission rate. When this is the case, the relative population emitting from the core state will be larger, as carriers will be unable to trap to the surface state before emitting. The surface:core population ratio is shown in Figure 4.5. Notably, the maximum in the surface:core ratio found in the experimental data is reflected in this model.

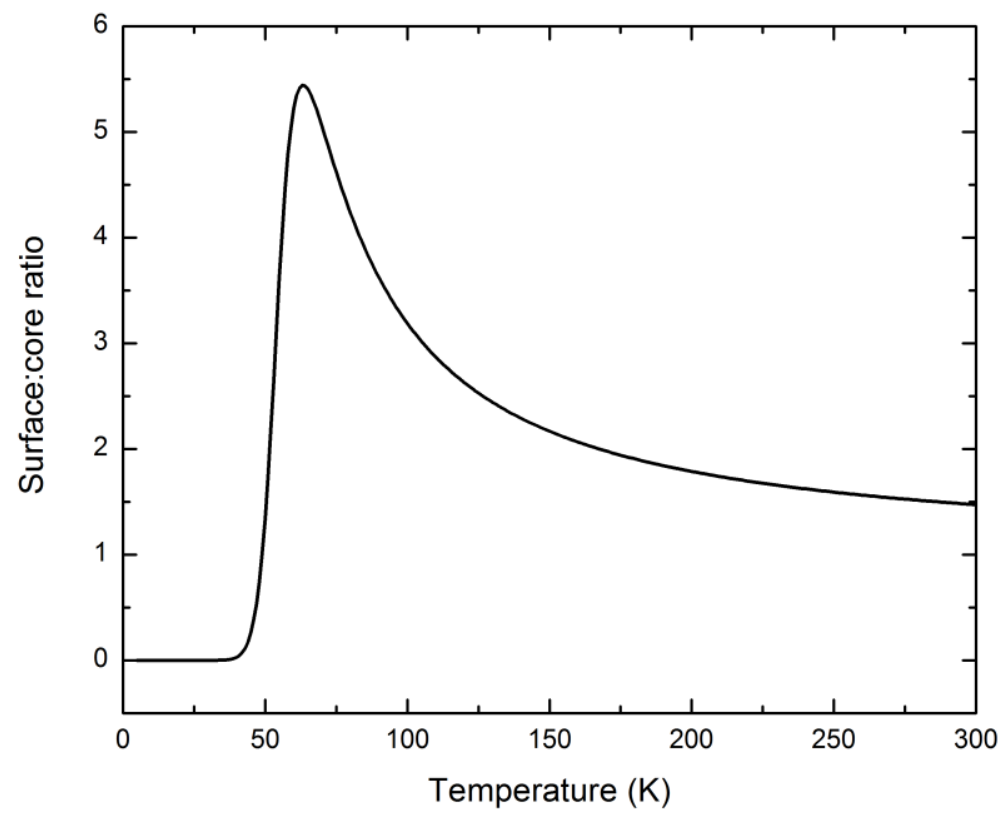

Figure 4.6. The ratio of surface:core emission shows a maximum at intermediate temperature but drops rapidly at low temperature when calculated using classical Marcus theory.

While this model makes significant progress in accounting for the observed phenomena, it contains some problems. Notably, the ratio of surface:core population is predicted to drop off exceedingly fast at low temperature, resulting in $100 \%$ of the population remaining in the core 
state at very low temperature. Such behaviour is not observed experimentally. In addition, only small values of both $\Delta \mathrm{G}^{0}$ and $\Delta \mathrm{G}^{\ddagger}$ on the order of $\mathrm{k}_{\mathrm{B}} \mathrm{T}$ can produce the required thermal exchange. Small values of both $\Delta \mathrm{G}^{0}$ and $\Delta \mathrm{G}^{\ddagger}$ are only possible at small values of coupling (reorganization energy), owing to the relationship in Equation 3.27:

$$
\Delta G^{\ddagger}=\frac{\left(\lambda-\Delta G^{0}\right)^{2}}{4 \lambda}
$$

But small values of coupling are inconsistent with the large redshift $(300-500 \mathrm{meV})$ and width $(300-500 \mathrm{meV})$ of surface emission. Hence the classical model, with the two states within $\sim \mathrm{k}_{\mathrm{B}} \mathrm{T}(<50 \mathrm{meV})$ as required to appreciably vary the relative populations (i.e. PL intensities), would fail to capture the observed broadening and redshifting $(\sim 300 \mathrm{meV})$ of surface emission (Fig 4.2d).

\subsection{Marcus-Jortner approach to surface trapping}

Marcus-Jortner semiclassical ET theory ${ }^{20}$, which incorporates both a classical, lowfrequency mode representing interaction with the medium, and quantum mechanical highfrequency mode representing internal vibrations, provides a model that can address these shortcomings. Within this theory, a low-frequency, classical mode dictates the temperaturedependence of PL via $\Delta G^{0}$ and $\Delta G^{\ddagger}$, while a high-frequency, quantum mode dictates lowtemperature behaviour via tunneling as well as spectral features such as redshift and width via coupling. By invoking a quantum-mechanical treatment of the internal mode, Marcus-Jortner theory allows transitions to occur via tunnelling, allowing a more satisfactory treatment of systems involving strong coupling that permits the necessarily low values of $\Delta \mathrm{G}^{0}$ and $\Delta \mathrm{G}^{\ddagger}$. 
To model the temperature-dependence of lineshape areas of the core and surface emission in the NC samples, a simple kinetic model based upon Marcus-Jortner electron-transfer between the core state and the surface state was developed. Three states are employed in the model: the ground state $\left(\mathrm{n}_{0}\right)$, the $1 \mathrm{~S}$ state $\left(\mathrm{n}_{1}\right)$, and the surface state $\left(\mathrm{n}_{2}\right)$ (Fig 4.3a). Electron transfer-like transitions from the 1S core state to the surface state and back were allowed according to the Marcus-Jortner ET rate equation (Equation 3.29) ${ }^{20}$ :

$$
\begin{gathered}
k_{E T \text { forward }}=\frac{2 \pi}{\hbar} H_{R P}{ }^{2}\left(\frac{\pi}{\hbar^{2} \lambda_{m} k_{b} T}\right)^{\frac{1}{2}} \sum_{v^{\prime}} e^{-S} \frac{S^{v^{\prime}}}{v^{\prime} !} e^{\frac{-\left(\Delta G^{0}+\lambda_{m}+v^{\prime} \hbar \omega\right)^{2}}{4 \lambda_{m} k_{b} T}} \\
k_{E T \text { back }}=\frac{2 \pi}{\hbar} H_{R P}{ }^{2}\left(\frac{\pi}{\hbar^{2} \lambda_{m} k_{b} T}\right)^{\frac{1}{2}} \sum_{v^{\prime}} e^{-S} \frac{S^{v^{\prime}}}{v^{\prime} !} e^{\frac{-\left(\Delta G^{0}+\lambda_{m}+v^{\prime} \hbar \omega\right)^{2}}{4 \lambda_{m} k_{b} T}} e^{\frac{\Delta G^{0}}{k_{b} T}}
\end{gathered}
$$

Here, $\Delta \mathrm{G}^{0}$ represents the energy difference between the $1 \mathrm{~S}$ and surface states and $\lambda_{\mathrm{m}}$ is the reorganization energy of the medium; these two factors govern the classically activated mode. The value $\mathrm{S}$ is the coupling of the internal quantum mode, in this case, the LO phonon due to its influence on the nanocrystal polarization (see Section 4.9); this factor governs the redshift and breadth of surface emission. The value $H_{R P}$ is the electronic matrix element, $\omega$ is the frequency of internal mode, and $v^{\prime}$ is the excited state vibrational quantum number. Excitation into the $1 \mathrm{~S}$ core exciton (as spectroscopically prescribed) is followed by emission from both the $1 \mathrm{~S}$ core excitonic and the surface state (Appendix B). 
If the ratio of surface to core population is again plotted vs. temperature but employing Marcus-Jortner theory to calculate transfer rates, a trend that is much more in line with the experimental data is produced (Figure 4.6). Here, the parameters $\Delta \mathrm{G}=10 \mathrm{meV}$ is used and the reorganization energy is partitioned into inner (quantum) and medium parts, with values of $\lambda_{m}=$ $20 \mathrm{meV}$ and $\mathrm{S}=21$. The oscillatory "bump" in the ratio at low temperature is easily accounted for. It has previously been observed that there is an oscillatory component to the electron transfer rate at low temperature under Marcus-Jortner theory, which is analogous to the vibrational structure in optical spectroscopy. These oscillations are fundamental, quantummechanical features that arise due to the vibrational structure of the system. They arise due to the quantum mode, somewhat analogous to the spectroscopic vibrational structure depicted in Figure 3.4. This phenomenon that has been referred to as "chemical type spectroscopy" in the literature. ${ }^{21}$ Clearly, Marcus-Jortner theory, which is better-suited to explaining electron transfer rates at low temperatures in which tunneling plays an important role, provides a better framework for understanding the experimental data.

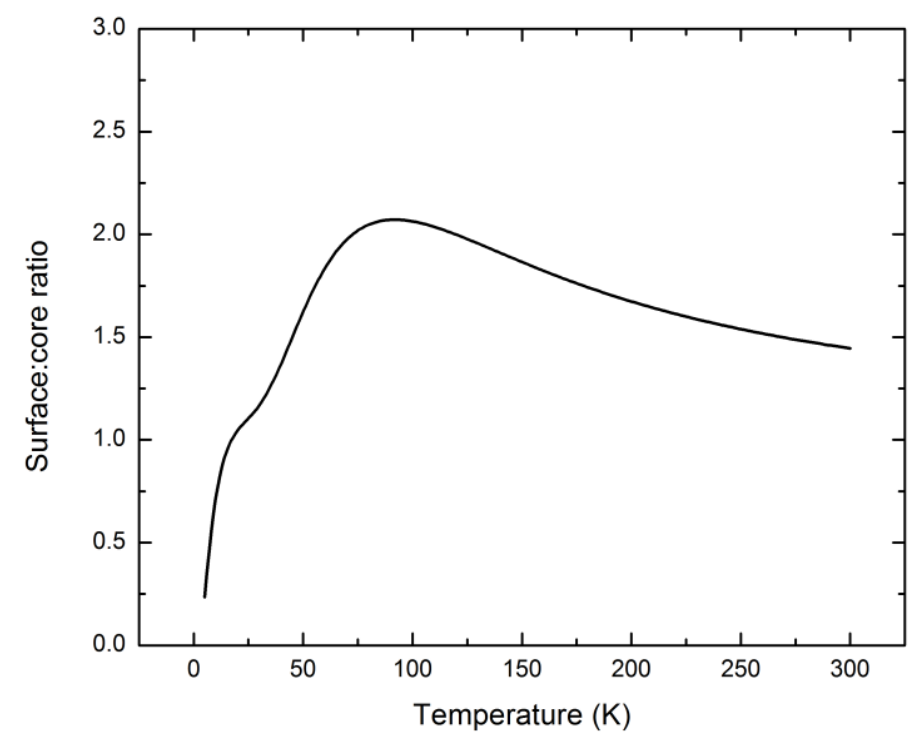


Figure 4.6. The ratio of surface:core emission shows is slowly varying and exhibits a maximum at intermediate temperature when calculated using semiclassical Marcus-Jortner theory.

As a first test of Marcus-Jortner theory's ability to account for the experimental data, empirical values of the ratio of surface to core emission at several temperatures were fit using equations above. The only adjustable parameters in this fit were the free energy difference $\Delta \mathrm{G}^{0}$, the reorganization energy $\lambda_{m}$, the electronic matrix element $H_{R P}$, and a constant accounting for the average number of trap sites per nanocrystal which affects the final density of electronic states and modulates $\mathrm{H}_{\mathrm{RP}}$ in the forward rate expression (4.2). The sum of $\mathrm{S} /\left(\hbar \omega_{\mathrm{LO}}\right)$ and $\Delta \mathrm{G}^{0}$ set equal to the energy difference between the two emission peaks as a constraint. Although Fig 4.1(c-d) clearly show that there are activated nonradiative pathways affecting both core and surface emission and the wide range of ratios observed experimentally suggests the possibility that some surface states do not emit, by using the ratio of surface to core emission as the quantity to fit, these factors can be accounted for in a single constant. 
a)

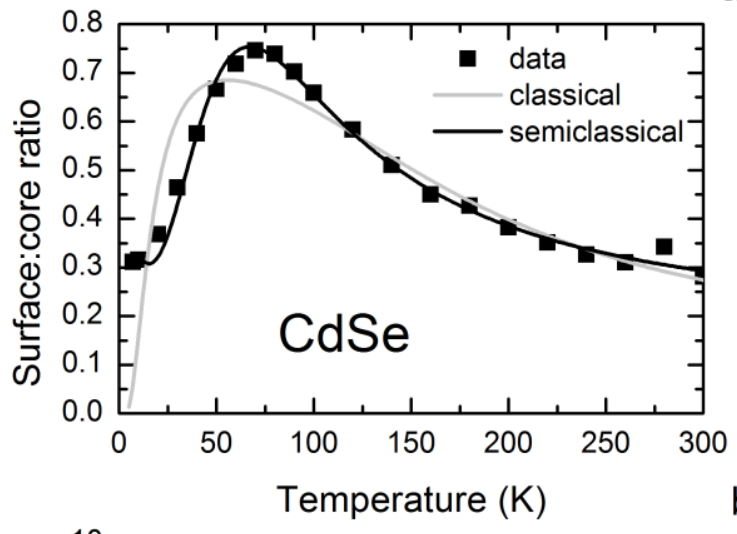

b)

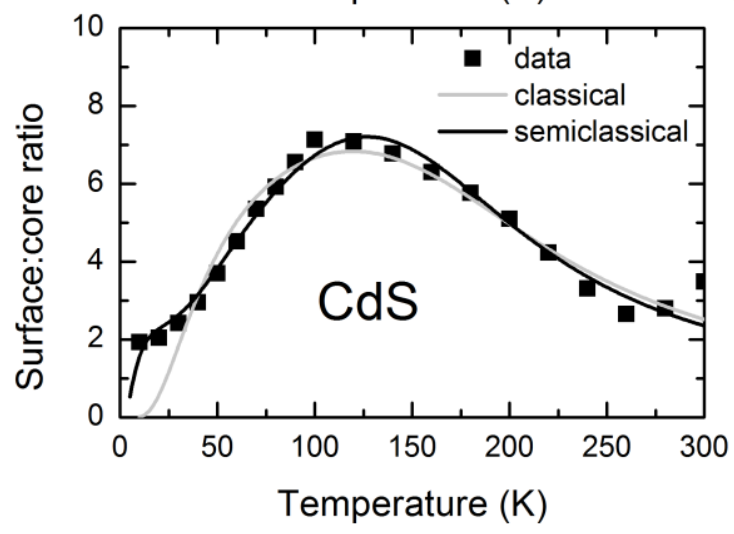

Figure 4.7. The experimental ratios of surface:core emission at several temperature are welldescribed by Marcus-Jortner electron transfer theory, while classical Marcus theory does not fit the data at low temperature. Samples are CdSe $(\mathrm{R}=1.15 \mathrm{~nm})(\mathrm{a})$ and $\mathrm{CdS}(\mathrm{R}=2.04 \mathrm{~nm})(\mathrm{b})$.

Fig 4.7 demonstrates the remarkable effectiveness of Marcus-Jortner electron transfer theory on accounting for the experimental data regarding the ratio of surface:core emission as a function of temperature. For both $\mathrm{CdSe}$ and $\mathrm{CdS}$ samples, the theory correctly predicts the peak in the ratio at intermediate temperature as well as the relatively smooth decrease in the ratio as temperature falls below this peak value. In terms of fit parameters, for CdSe the fit results in values of $\Delta \mathrm{G}=18 \pm 2 \mathrm{meV}, \lambda=14 \pm 2 \mathrm{meV}$, and $\mathrm{S}=22 \pm 3$, while for CdS the parameters are of $\Delta \mathrm{G}=62 \pm 7 \mathrm{meV}, \lambda=16 \pm 7 \mathrm{meV}$, and $\mathrm{S}=21 \pm 16$ (See Appendix B). 


\subsection{Marcus-Jortner electron transfer: a more complete picture}

A more complete model would be able to account not only for the trends in the ratio of surface:core emission but also for the unique temperature-dependence of each type of emission itself. It is clear from the data that there is a strong temperature-dependence to the quantum yield for both emission channels. The simplest way to account for this decrease in emission with temperature is to include a single activated, nonradiative decay channel that affects all NCs. Such a channel would compete with decay through emission, thereby reducing the percentage of carriers that emit in a temperature-dependent fashion. If such a decay channel were present, it would be expected to affect both the quantum yield and the observed lifetime of the NCs due to the following relations:

$$
\begin{gathered}
\Phi(T)=\frac{k_{\text {rad }}}{k_{\text {observed }}(T)} \\
k_{\text {observed }}(T)=k_{\text {rad }}+k_{n r}(T)
\end{gathered}
$$

Here $\Phi(T)$ is the quantum yield, $k_{\text {observed }}(T)=\frac{1}{\tau_{\text {observed }}(T)}$ is the reciprocal of the observed lifetime, $\mathrm{k}_{\mathrm{rad}}$ is the intrinsic radiative lifetime, and $k_{n r}(T)$ is the nonradiative decay rate which might take the form $\frac{1}{\tau_{n r}} e^{\frac{-E_{a}}{k_{B} T}} \cdot{ }^{22}$ Here $E_{a}$ is an activation energy representing the barrier to nonradiative emission and $\tau_{n r}$ is a parameter that accounts for the probability of this process. 
Despite the strong temperature-dependence to the quantum yield in these samples, our results and those of others show that the observed lifetimes of the NCs over the same temperature range are relatively independent of temperature. ${ }^{23,24}$ Such a result suggests a basic inconsistency between equations 4.4 and 4.5 .

This inconsistency can be resolved by considering that the system of NCs is not homogeneous. Considerable recent work has suggested that the NCs within an ensemble are far from homogeneous in their decay pathways. ${ }^{23,25-27}$ Thus, we propose that the percentage of NCs within the ensemble which emit in the first place is a temperature-dependent quantity, $\mathrm{n}(\mathrm{T})$. Hence the ensemble quantum yield has a temperature-dependence which now includes the fraction of NCs which emit,

$$
\Phi(T)=\frac{k_{r a d}}{k_{r a d}+k_{n r}} n(T)
$$

Thus, the loss of quantum yield at higher temperature does not result from a thermally activated nonradiative decay pathway common to all NCs, but rather from a fraction of NCs within the ensemble ceasing to emit at higher temperatures.

In this model, the fraction of NCs which emit via surface and core emission each take on a unique temperature-dependence:

$$
\begin{gathered}
\text { Fraction of core emitting NCs }=1-A_{C} e^{\frac{-E a_{C}}{k_{b} T}} \\
\text { Fraction of surface emitting } N C S=1-A_{S} e^{\frac{-E a_{S}}{k_{b} T}}
\end{gathered}
$$


Here $E_{a c}$ and $E_{a c}$ are the activation energies to nonradiative emission from the core and surface of the nanocrystal, respectively, while $A_{C}$ and $A_{S}$ are weighting factors representing the probability of these processes. The $\left(1-A e^{\frac{-E a}{k_{b} T}}\right)$ format of the equations physically describe a system in which $100 \%$ of the nanocrystals emit at $0 \mathrm{~K}$ and smaller fractions emit from the core and surface as temperature is increased. The room temperature value of quantum yield was measured as $\sim 10 \%$ for samples of CdSe.

These equations represent, necessarily, a somewhat phenomenological means of accounting for the observed data (See Appendix B for a discussion of alternate approaches). The question of nonradiative decay pathways in NCs is extraordinarily complicated. For example, measured lifetimes are typically highly multiexponential, and this multiexponental character has been shown to result from the complexity of decay even on the single-dot level. ${ }^{28}$ Efforts to account for nonradiative pathways even at a single temperature have resulted in very complex models. ${ }^{26}$ Thus, since the focus of this work is the nature of carrier trapping and surface emission rather than the complex question of nonradiative decay pathways, the above equations are appropriate since they are both simple, in the sense that they incorporate few floating parameters, and consistent with all observed data, in the sense that they account for both the strong temperature-dependence of the quantum yield and the unexpected temperatureindependence of the lifetime.

The PL decays obtained from time-correlated single photon counting (TCSPC) measurements of core and surface emission were found to be multiexponential, in line with previous results. While many methods have been used to estimate lifetimes from these multiexponential decays ${ }^{23}$, here the entire decay was deconvoluted from the instrument response function (IRF) and fit to a triexponential to produce acceptable $\chi^{2}$ values. The fit algorithm was 
the FuloFit nonlinear least-squares error minimization based on the Levenberg-Marquardt algorithm. The intensity-weighted average lifetime of the core and surface emission decays calculated was calculated from this fit. At room temperature, lifetimes of $25.1 \mathrm{~ns}$ for core emission and $33.8 \mathrm{~ns}$ for surface emission were obtained this way for liquid samples of CdSe NCs. The value obtained for core emission is in line with previous results, as is the ratio of core to surface lifetimes. ${ }^{29}$ When samples were placed in polymer film and lifetimes were measured at room temperature and $77 \mathrm{~K}$, lifetime values of 2.4 and $2.5 \mathrm{~ns}$ were obtained, indicating a variation of less than $5 \%$. Thus, lifetimes remained relatively unchanged with temperature. The lifetimes obtained in solution rather than were used in modeling because the signal obtained from the film was weak and potentially influenced by scatter.

With these factors in mind, the data for both core and surface lineshape areas were simultaneously fitted using the equations above. A nonlinear least-squares regression analysis of the experimental data to the model equations results in the fits shown as solid lines in Fig. 4.1c-f. Clearly, the model produces excellent fits to the data. The fit parameters for CdSe are $\Delta \mathrm{G}=13 \pm$ $1 \mathrm{meV}, \lambda=8 \pm 1 \mathrm{meV}$, and $23 \pm 2$, while for $\mathrm{CdS}$ they are $\Delta \mathrm{G}=66 \pm 7 \mathrm{meV}, \lambda=21 \pm 8 \mathrm{meV}$, and $21 \pm 18$ (See Appendix B for details). Figure 4.1e-f shows that in particular the model can accurately capture the maxima of the surface:core ratio at intermediate temperature for a variety of samples.

\subsection{Modeling spectra from output data}

Clearly the temperature-dependence of the PL lineshape band areas are well fit by this approach featuring two modes. Yet any complete model must also correctly account for the 
breadth $(\Delta \mathrm{E})$ and redshifting $(\delta \mathrm{E})$ of the surface PL. In order to further test the robustness of the model, PL spectra were simulated using the same output parameters (e.g. S, $\Delta \mathrm{G}$, etc.) obtained with the above model.

The wavepacket approach of Heller et al. involves the Fourier transform of the dipoledipole time-correlation function. ${ }^{36}$ This function was used to simulate the PL spectra at several temperatures:

$$
I_{T}(\omega)=\int_{-\infty}^{\infty}\langle 0 \mid 0(t)\rangle e^{i \omega t-\frac{1}{2} \Theta^{2} t^{2}-\Gamma|t|}
$$

Here $\langle f \mid i(t)\rangle$ is the correlation function, $\omega$ is the excitation frequency, $\Theta$ is an inhomogeneous broadening parameter, and $\Gamma$ is the homogeneous linewidth. The importance of these broadening parameters is demonstrated in Fig 4.4, where spectra are shown with them both included and excluded. The homogeneous linewidth, which depends on collisional broadening, is temperature-dependent. In the case of harmonic potential energy surfaces:

$$
\langle 0 \mid 0(t)\rangle=\prod_{k} e^{-S_{k} \cdot\left(1-e^{-i \omega_{k} t}\right)-i \omega_{e g} t}
$$

Where $\mathrm{S}$ is the Huang-Rhys parameter, $\omega_{\mathrm{k}}$ is the phonon energy, and $\omega_{\mathrm{eg}}$ represents the energy difference between the ground and excited state potential energy surface minima.

In these simulations, based on the literature regarding the temperature-dependence of the homogeneous linewidth of CdSe NCs, the value $\Gamma$ was varied linearly from $20 \mathrm{meV}$ at $20 \mathrm{~K}$ to $50 \mathrm{meV}$ at $300 \mathrm{~K}^{30-34} \mathrm{~A}$ value of $20.7 \mathrm{meV}$ was employed for $\Theta$ based on the best fit for the 
lowest-temperature data. The core and surface spectra were simulated individually, with the peak energy based upon fitting PL data to the Varsnhi equation ${ }^{3}$ for the core peak and based on the values of $\mathrm{S}_{\text {surface }}$ and $\Delta \mathrm{G}_{\text {core-surface }}^{0}$ for the surface peak. The intensities of the peaks were modeled using the output of the temperature-dependent kinetic model, with all values normalized to the core peak at $140 \mathrm{~K}$. The results of these simulations are shown in Fig. 4.8. The output parameters of the model produce simulated spectra in good qualitative accord with the experimental data. 
a)

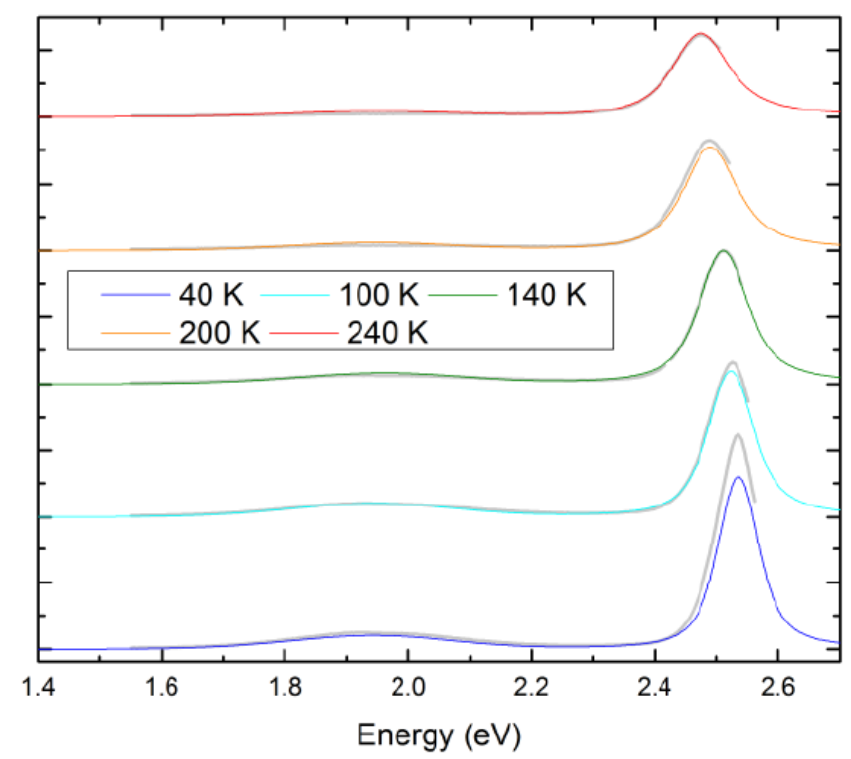

b)

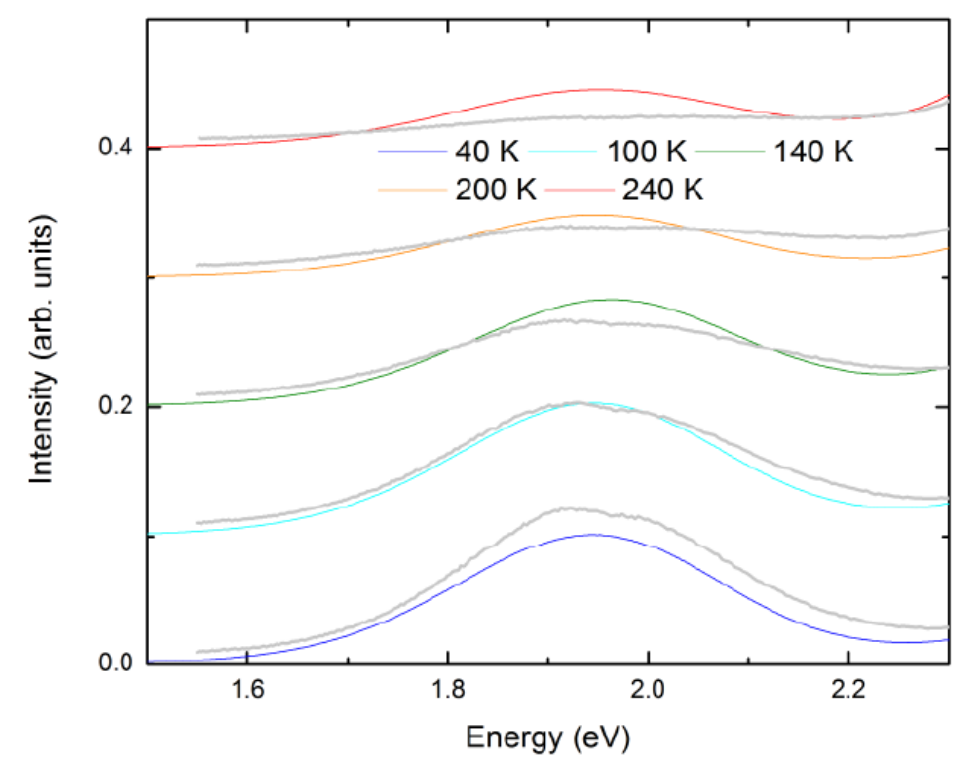

Figure 4.8. Spectra simulated using the output parameters from fitting using Marcus-Jortner electron transfer theory using Heller et al.'s approach are in good accord with the experimental data $(\mathrm{R}=1.15 \mathrm{~nm} \mathrm{CdSe})$. The colored lines show the simulations and the light grey lines show the data. Panel a) shows the full spectra, while panel b) shows highlights the surface emission. 


\subsection{Discussion}

Several additional pieces of evidence support the model of surface emission outlined in this chapter. Recent work has demonstrated inherently broad (200-400 meV) surface-related emission from individual NCs. ${ }^{35,36}$ Such a result suggests that, if the deep-trap model of the surface involving an energetic distribution of states is valid, this distribution is present on each NC. Yet the overall shape of the surface peak remains largely unchanged with temperature (Fig. 4.1a). As first noted by Banin's group ${ }^{37}$, if surface emission arose from a distribution of states within individual NCs, a redshift in the spectrum would be expected at low temperature owing to insufficient thermal energy to populate higher states. No such redshift is observed, lending further support to the phonon-based understanding of surface emission presented here.

In addition, the shape and width of the surface emission spectrum does not change when fluorescence line-narrowing is employed to select a subset of the largest NCs in the ensemble, nor does the photoluminescence excitation (PLE) spectrum change based on the choice of emission energy within the surface peak. All these results suggest that phonon coupling, rather than a distribution of trap states, is responsible for the breadth of surface emission.

One of the criticisms that has been offered of a model in which the same NCs give rise to both core and surface emission is the fast rise time observed for emission from the surface state. Bawendi et al. noted that a slower rise time would be expected if the band edge emission were feeding the deep trap emission, but that the two rise times were not resolvable $(<500 \mathrm{ps})$ in their experiment. ${ }^{38}$ Thus, they proposed that two separate populations were responsible for the two types of emission. However, internal conversion is known to happen on a very fast timescale $(<1$ ps) $)^{39}$ and Rosenthal's group would later find through an experiment with better time-resolution that the deep trap emission grows as the band edge emission decays. ${ }^{40}$ 


\subsection{The nature of the surface state}

The model provides a good fit to the temperature dependence of the lineshape areas over all temperatures. The reorganization energy $\lambda_{\text {quantum }}$ and thus $\mathrm{S}$ of the quantum mode is large (e.g. $\mathrm{S}=23 \pm 5$ for $\mathrm{R}=1.15 \mathrm{~nm} \mathrm{CdSe}$ ), leading to strong displacement and a vibronic progression in the surface emission (Fig. $4.2 \mathrm{f}$ and 4.3) that accounts for its width and shift. In contrast, $\lambda_{\mathrm{m}}$ and $\Delta \mathrm{G}^{0}$ are small (e.g. $14 \pm 2$ and $13 \pm 1 \mathrm{meV}$ respectively for $\mathrm{R}=1.15 \mathrm{~nm} \mathrm{CdSe}$ ) for the classical mode, allowing for the thermally activated population exchange that explains the temperaturedependence of the surface emission. These results suggest that the very broad surface emission in semiconductor NCs can be understood as emanating from a single, strongly coupled surface state following an electron-transfer-like transition of a charge carrier from the core state of the NC. The physics of a strongly coupled surface state can be explained by considering the polarization change that is created upon surface charge trapping.

In polar semiconductors, charge carriers couple to Longitudinal Optical (LO) phonons via the polar or Fröhlich interaction. Upon localization of one of the charge carriers at the surface of the nanocrystal, the nanocrystal becomes substantially more polarized and the strength of this coupling increases, explaining the large coupling obtained. With regard to bath interaction, Jones and Scholes have previously identified bath polarization as the reaction coordinate in their classical Marcus ET treatment of surface trapping by demonstrating a correlation between reorganization energy and solvent polarizibility. ${ }^{9}$ Hence polarization is identified as the reaction coordinate in this system.

Notably, this is not the first work to suggest that strong coupling and a phonon progression could be responsible for the broad surface emission. In 1986, Chestnoy et al. 
examined the broad luminescence in CdS nanocrystals. ${ }^{41}$ They proposed that a major factor contributing to broad emission was strong coupling to lattice vibrations, creating a phonon progression, which was further broadened due to Coulomb interaction and a distribution of trap depths. In 1998, Lifshitz analyzed NCs prepared by chemical solution deposition and sol-gel deposition. Noting the unique temperature-dependence for the core and surface emission and the breadth of surface emission, Lifshitz analyzed the results in the configuration coordinate picture and argued that the surface emission width arose from strong coupling to lattice phonons, specifically involving a deep-trapped hole due to its larger effective mass and the shifting of the spectrum upon saturating distant pairs. ${ }^{13}$ In that work, a phenomenological explanation for the temperature-dependence was offered. Despite these advances, the explanation of strong coupling and a phonon progression is rarely invoked in the literature to explain the properties of surface emission. In addition, the two works cited that offered this explanation did not provide a microscopic explanation for the temperature dependence of the two emissive states, as is offered here via Marcus-Jortner electron transfer theory. Thus, this work is the first to tie together disparate findings to offer a complete picture of nanocrystal surface trapping.

In summary, the high value of electron-phonon coupling for the surface state which leads to a phonon progression in the photoluminescence spectrum and thus the redshifting and breadth of the surface emission (Fig. 4.3). These results show that NCs possess a surface state with a well-defined energy that is on the order of kT below the $1 \mathrm{~S}$ core state and strongly coupled via phonons, and that thermal population exchange is possible between this state and the 1S core state. 


\section{References}

1. Yu, W. W.; Qu, L.; Guo, W.; Peng, X., Experimental Determination of the Extinction Coefficient of CdTe, CdSe, and CdS Nanocrystals. Chem Mater 2003, 15, 2854-2860.

2. Kuno, M.; Lee, J. K.; Dabbousi, B. O.; Mikulec, F. V.; Bawendi, M. G., The band edge luminescence of surface modified CdSe nanocrystallites: Probing the luminescing state. $J$ Chem Phys 1997, 106, 9869-9882.

3. Valerini, D.; Creti, A.; Lomascolo, M.; Manna, L.; Cingolani, R.; Anni, M., Temperature dependence of the photoluminescence properties of colloidal $\mathrm{CdSe} / \mathrm{ZnS}$ core/shell quantum dots embedded in a polystyrene matrix. Phys Rev B 2005, 71, 235409.

4. Kambhampati, P., Hot Exciton Relaxation Dynamics in Semiconductor Quantum Dots: Radiationless Transitions on the Nanoscale. J Phys Chem C 2011, 115, 22089-22109.

5. Tyagi, P.; Kambhampati, P., False multiple exciton recombination and multiple exciton generation signals in semiconductor quantum dots arise from surface charge trapping. $J C h e m$ Phys 2011, 134, 094706-10.

6. $\quad$ Tyagi, P.; Cooney, R. R.; Sewall, S. L.; Sagar, D. M.; Saari, J. I.; Kambhampati, P., Controlling Piezoelectric Response in Semiconductor Quantum Dots via Impulsive Charge Localization. Nano Lett 2010, 10, 3062-3067.

7. $\quad$ Cooney, R. R.; Sewall, S. L.; Sagar, D. M.; Kambhampati, P., State-Resolved Manipulations of Optical Gain in Semiconductor Quantum Dots: Size Universality, Gain Tailoring, and Surface Effects. J Chem Phys 2009, 131, 164706.

8. $\quad$ Sewall, S. L.; Cooney, R. R.; Anderson, K. E. H.; Dias, E. A.; Sagar, D. M.; Kambhampati, P., State-resolved studies of biexcitons and surface trapping dynamics in semiconductor quantum dots. J Chem Phys 2008, 129, 084701.

9. Jones, M.; Lo, S. S.; Scholes, G. D., Quantitative modeling of the role of surface traps in $\mathrm{CdSe} / \mathrm{CdS} / \mathrm{ZnS}$ nanocrystal photoluminescence decay dynamics. P Natl Acad Sci USA 2009, 106, 3011-3016.

10. Kortan, A. R.; Hull, R.; Opila, R. L.; Bawendi, M. G.; Steigerwald, M. L.; Carroll, P. J.; Brus, L. E., Nucleation and growth of cadmium selendie on zinc sulfide quantum crystallite seeds, and vice versa, in inverse micelle media. J Am Chem Soc 1990, 112, 1327-32.

11. Hasselbarth, A.; Eychmuller, A.; Weller, H., Detection of Shallow Electron Traps in Quantum Sized Cds by Fluorescence Quenching Experiments. Chem Phys Lett 1993, 203, 271 276.

12. Jones, M.; Nedeljkovic, J.; Ellingson, R. J.; Nozik, A. J.; Rumbles, G., Photoenhancement of Luminescence in Colloidal CdSe Quantum Dot Solutions. J Phys Chem B 2003, 107, 11346-11352.

13. Lifshitz, E.; Dag, I.; Litvin, I.; Hodes, G.; Gorer, S.; Reisfeld, R.; Zelner, M.; Minti, H., Properties of CdSe nanoparticle films prepared by chemical deposition and sol-gel methods. Chem Phys Lett 1998, 288, 188-196.

14. Babentsov, V.; Sizov, F., Defects in quantum dots of IIB-VI semiconductors. OptoElectronics Review 2008, 16, 208-225.

15. Trindade, T.; OBrien, P.; Zhang, X. M., Synthesis of CdS and CdSe nanocrystallites using a novel single-molecule precursors approach. Chem Mater 1997, 9, 523-530. 
16. Mooney, J.; Kambhampati, P., Get the Basics Right: Jacobian Conversion of Wavelength and Energy Scales for Quantitative Analysis of Emission Spectra. J Phys Chem Lett 2013, 4, 3316-3318.

17. Krause, M. M.; Mooney, J.; Kambhampati, P., Chemical and Thermodynamic Control of the Surface of Semiconductor Nanocrystals for Designer White Light Emitters. Acs Nano 2013, 7, 5922-5929.

18. Spanhel, L.; Haase, M.; Weller, H.; Henglein, A., Photochemistry of Colloidal Semiconductors .20. Surface Modification and Stability of Strong Luminescing Cds Particles. $J$ Am Chem Soc 1987, 109, 5649-5655.

19. Brus, L., Model for carrier dynamics and photoluminescence quenching in wet and dry porous silicon thin films. Phys Rev B 1996, 53, 4649-4656.

20. Jortner, J., Temperature-Dependent Activation-Energy for Electron-Transfer between Biological Molecules. J Chem Phys 1976, 64, 4860-4867.

21. Ulstrup, J.; Jortner, J., Effect of Intramolecular Quantum Modes on Free-Energy Relationships for Electron-Transfer Reactions. J Chem Phys 1975, 63, 4358-4368.

22. Valerini, D.; Creti, A.; Lomascolo, M.; Manna, L.; Cingolani, R.; Anni, M., Temperature dependence of the photoluminescence properties of colloidal $\mathrm{CdSe} / \mathrm{ZnS}$ core/shell quantum dots embedded in a polystyrene matrix. Phys Rev B 2005, 71.

23. Crooker, S. A.; Barrick, T.; Hollingsworth, J. A.; Klimov, V. I., Multiple temperature regimes of radiative decay in $\mathrm{CdSe}$ nanocrystal quantum dots: Intrinsic limits to the dark-exciton lifetime. Appl Phys Lett 2003, 82, 2793-2795.

24. Donega, C. D.; Bode, M.; Meijerink, A., Size- and temperature-dependence of exciton lifetimes in CdSe quantum dots. Phys Rev B 2006, 74.

25. Ebenstein, Y.; Mokari, T.; Banin, U., Fluorescence quantum yield of CdSe/ZnS nanocrystals investigated by correlated atomic-force and single-particle fluorescence microscopy. Appl Phys Lett 2002, 80, 4033-4035.

26. Knowles, K. E.; McArthur, E. A.; Weiss, E. A., A Multi-Timescale Map of Radiative and Nonradiative Decay Pathways for Excitons in CdSe Quantum Dots. Acs Nano 2011, 5, 20262035.

27. Kern, S. J.; Sahu, K.; Berg, M. A., Heterogeneity of the Electron-Trapping Kinetics in CdSe Nanoparticles. Nano Lett 2011, 11, 3493-3498.

28. Fisher, B. R.; Eisler, H. J.; Stott, N. E.; Bawendi, M. G., Emission intensity dependence and single-exponential behavior in single colloidal quantum dot fluorescence lifetimes. $J$ Phys Chem B 2004, 108, 143-148.

29. Baker, D. R.; Kamat, P. V., Tuning the Emission of CdSe Quantum Dots by Controlled Trap Enhancement. Langmuir 2010, 26, 11272-11276.

30. Salvador, M. R.; Hines, M. A.; Scholes, G. D., Exciton-bath coupling and inhomogeneous broadening in the optical spectroscopy of semiconductor quantum dots. $J$ Chem Phys 2003, 118, 9380-9388.

31. Schoenlein, R. W.; Mittleman, D. M.; Shiang, J. J.; Alivisatos, A. P.; Shank, C. V., Investigation of femtosecond electronic dephasing in cadmium selenide nanocrystals using quantum-beat-suppressed photon echoes. Phys Rev Lett 1993, 70, 1014-17.

32. Bawendi, M. G.; Wilson, W. L.; Rothberg, L.; Carroll, P. J.; Jedju, T. M.; Steigerwald, M. L.; Brus, L. E., Electronic structure and photoexcited-carrier dynamics in nanometer-size cadmium selenide clusters. Phys Rev Lett 1990, 65, 1623-6. 
33. Sun, Y.; Masia, F.; Langbein, W.; Borri, P., Fabrication and optical properties of thin silica-coated CdSe/ZnS quantum dots. Physica Status Solidi a-Applications and Materials Science 2009, 206, 2822-2825.

34. Müller, J.; Lupton, J. M.; Rogach, A. L.; Feldmann, J.; Talapin, D. V.; Weller, H., Monitoring Surface Charge Movement in Single Elongated Semiconductor Nanocrystals. Phys Rev Lett 2004, 93, 167402.

35. Dukes, A. D.; Samson, P. C.; Keene, J. D.; Davis, L. M.; Wikswo, J. P.; Rosenthal, S. J., Single-Nanocrystal Spectroscopy of White-Light-Emitting CdSe Nanocrystals. J Phys Chem A 2011, 115, 4076-4081.

36. Layek, A.; De, S.; Thorat, R.; Chowdhury, A., Spectrally Resolved Photoluminescence Imaging of ZnO Nanocrystals at Single-Particle Levels. J Phys Chem Lett 2011, 2, 1241-1247.

37. Kan, S. H.; Aharoni, A.; Mokari, T.; Banin, U., Shape control of III-V semiconductor nanocrystals: Synthesis and properties of InAs quantum rods. Faraday Discuss 2004, 125, 23-38. 38. Bawendi, M. G.; Carroll, P. J.; Wilson, W. L.; Brus, L. E., Luminescence Properties of Cdse Quantum Crystallites - Resonance between Interior and Surface Localized States. $J$ Chem Phys 1992, 96, 946-954.

39. Blanchet, V.; Zgierski, M. Z.; Seideman, T.; Stolow, A., Discerning vibronic molecular dynamics using time-resolved photoelectron spectroscopy. Nature 1999, 401, 52-54.

40. Underwood, D. F.; Kippeny, T.; Rosenthal, S. J., Ultrafast carrier dynamics in CdSe nanocrystals determined by femtosecond fluorescence upconversion spectroscopy. $J$ Phys Chem $B$ 2001, 105, 436-443.

41. Chestnoy, N.; Harris, T. D.; Hull, R.; Brus, L. E., Luminescence and Photophysics of Cds Semiconductor Clusters - the Nature of the Emitting Electronic State. Journal of Physical Chemistry 1986, 90, 3393-3399. 


\section{Chapter 5: Coupling strength and hot carrier effects}

*This chapter was partially adapted from Mooney, Jonathan, et al. "A microscopic picture of surface charge trapping in semiconductor nanocrystals." The Journal of chemical physics 138.20 (2013): 204705.

\subsection{Introduction}

This chapter builds upon the model developed in the prior chapter to explore its relationship to additional experiments. First, values of coupling obtained from the MarcusJortner model are compared to those obtained using resonance Raman spectroscopy as a test of consistency. Next, the trapping model developed in the prior chapter is applied to higher excited states in an effort to explain the results of other experiments investigating hot carrier trapping.

\subsection{Measurements of electron-phonon coupling}

In the previous chapter, it was shown that surface emission in nanocrystals could be explained as arising from a surface state with a large electron-phonon coupling $(\mathrm{S} \approx 20)$. To explore whether such large values of coupling are reasonable, a brief review of experiments which have measured values of coupling for the core and surface states of NCs is in order to ensure consistency with the coupling values obtained. We have previously shown ${ }^{1,2}$ that ultrafast measurements (See Appendix A for details) of frequency modulation in NC absorption due to coherent excitation of phonons provide a measure of the electron-phonon coupling 
strength for core states. ${ }^{3}$ Specifically, the amplitude $A_{\text {osc }}$ of these oscillations reflects the underlying coupling strength, $\Delta \omega$ :

$$
A_{o s c}=\left(\frac{d O D}{d \omega}\right) \Delta \omega
$$

Here $O D$ represents the optical density. From this expression, the coupling value $\mathrm{S}$ (Huang-Rhys parameter) can be easily determined:

$$
S=\frac{\Delta \omega}{\hbar \omega_{\text {phonon }}}
$$

Monitoring absorption at the rising edge of the $1 \mathrm{~S}$ feature produces maximum oscillations due to the magnitude of $\Delta \mathrm{OD} / \Delta \omega$ at this part of the spectrum. Fig. 5.1a. shows raw data on transient absorbance as a function of time monitored here. The non-oscillatory features in this spectrum are due to electronic effects such as state filling ${ }^{4}$ and level shifting ${ }^{4}$. The oscillatory features reflect coherent phonons that are launched when a sufficiently broad pulse excites multiple vibrational states, creating a wavepacket that evolves in time along a potential energy surface. ${ }^{2,5}$ Since the oscillations arise due to the propagation of the excited state wavepacket, the greater the coupling strength, the stronger the oscillations, allowing coupling strength to be determined spectroscopially. Fig. 5.1b. shows the data processed to extract the non-oscillatory features via fitting the transient after the arrival of the coherent pump pulse to a monoexponential decay and extracting the residuals. Two oscillatory components are visible in the residuals: a rapid oscillation due to optical phonons and a low-frequency oscillation due to acoustic phonons. Fig. 5.1c. shows the Fourier transform of these residuals to extract the amplitude of the oscillations. By measuring the maximum peak to trough amplitude of the phonons, the value of coupling to optical phonons for the core state is roughly estimated to be $\mathrm{S} \approx 0.013$. 

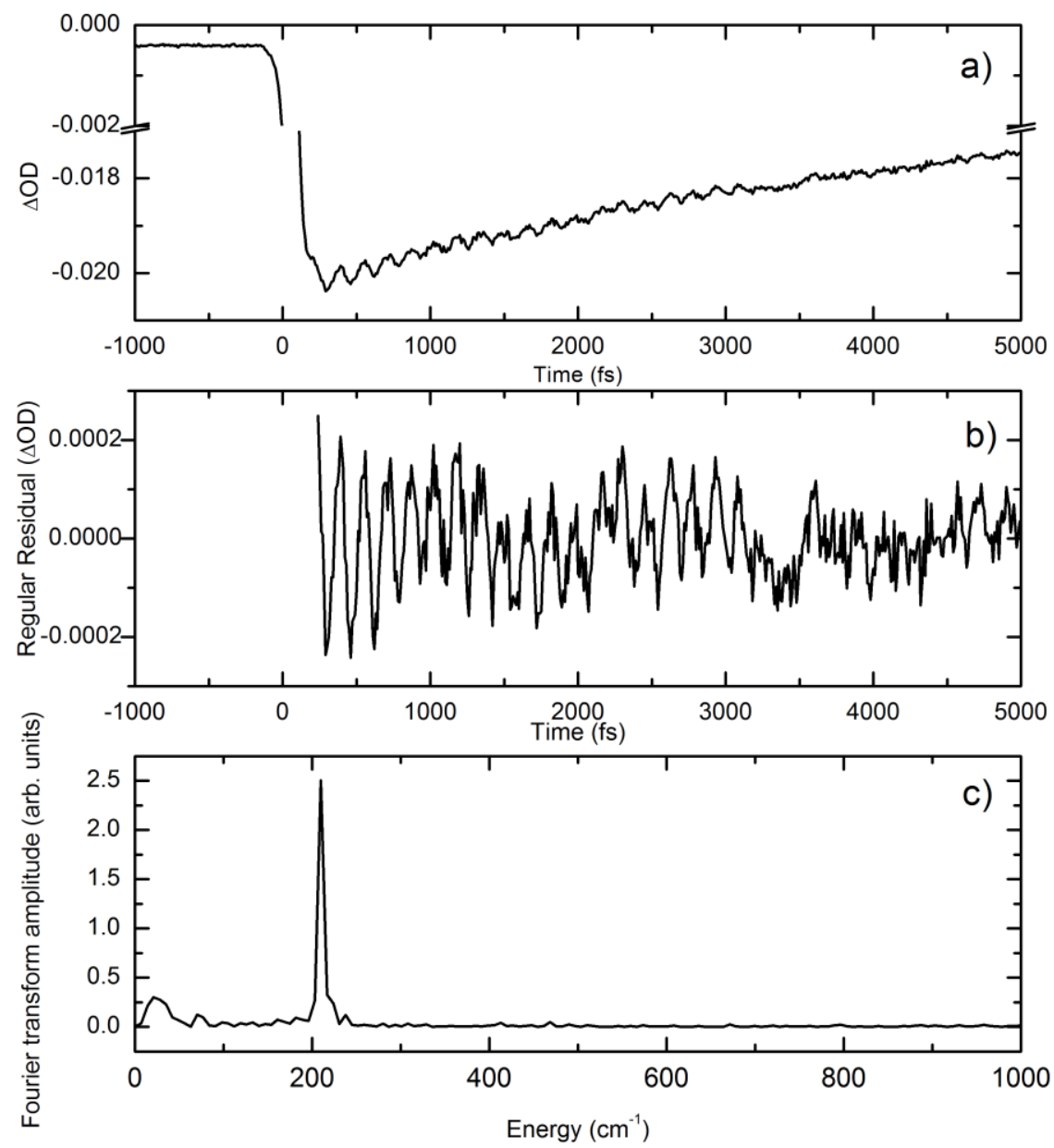

Figure 5.1. a) Coherent phonons present in the transient absorption spectrum of CdSe NCs. b) Removing the non-oscillatory component due to electronic effects shows the features due to phonons only. c) Amplitudes within the FFT of the oscillatory data provide a measure of electron-phonon coupling strength for the core state.

On the other hand, we and others ${ }^{1,6-8}$ have argued that continuous-wave experiments such as Raman experiments measure the coupling to surface-trapped excitons. As mentioned previously, within ET theory, coupling is modeled as the displacement of an excited state potential energy surface along a harmonic normal-mode coordinate. Thus, Heller's wavepacket 
approach is consistent with this model and can be used to model absorption, photoluminescence, and Raman excitation spectra. ${ }^{9}$ Within Heller's approach, the displacement (coupling) $\mathrm{S}_{\mathrm{k}}$ of mode $\mathrm{k}$ appears explicitly within the expressions for modeling the spectra. Thus a PL or Raman excitation spectrum can be used to predict the value of coupling and vice versa.

Resonance Raman spectra were obtained on CdSe NCs (Fig. 5.2). Background subtraction of the slowly varying fluorescence signal was performed by fitting this signal within the raw data to a fifth-order polynomial and subtracting it out to isolate the Raman signal. The background-subtracted data is presented in Fig. 5.2. The fundamental peak corresponding to the longitudinal optical (LO) phonon is clearly visible in the spectrum at $\sim 207 \mathrm{~cm}^{-1}$ while the overtone appears at $\sim 413 \mathrm{~cm}^{-1}$ (the second overtone is barely visible at $\sim 623 \mathrm{~cm}^{-1}$ ). The fundamental and first overtone peaks were simultaneously fitted to Voigt functions. Previous studies ${ }^{10,11}$ have demonstrated that in principle the ratio of overtone to fundamental intensity can be measured either from the Raman excitation profile or from the Raman spectrum directly if measured at the Raman excitation peak. Here, the Raman spectrum is used but explicitly the variation of this ratio as a function of Raman excitation energy is accounted for in determining the ratio. First, the contribution from the surface optical (SO) phonon component on the leading edge of the Raman spectrum is removed (Fig. 5.2), following the method previously established ${ }^{12-14}$ in the literature. Once removed, the remaining peaks, located at $206 \mathrm{~cm}^{-1}$ and $410 \mathrm{~cm}^{-1}$ in the fits, yield an overtone:fundamental ratio of 0.331 . 


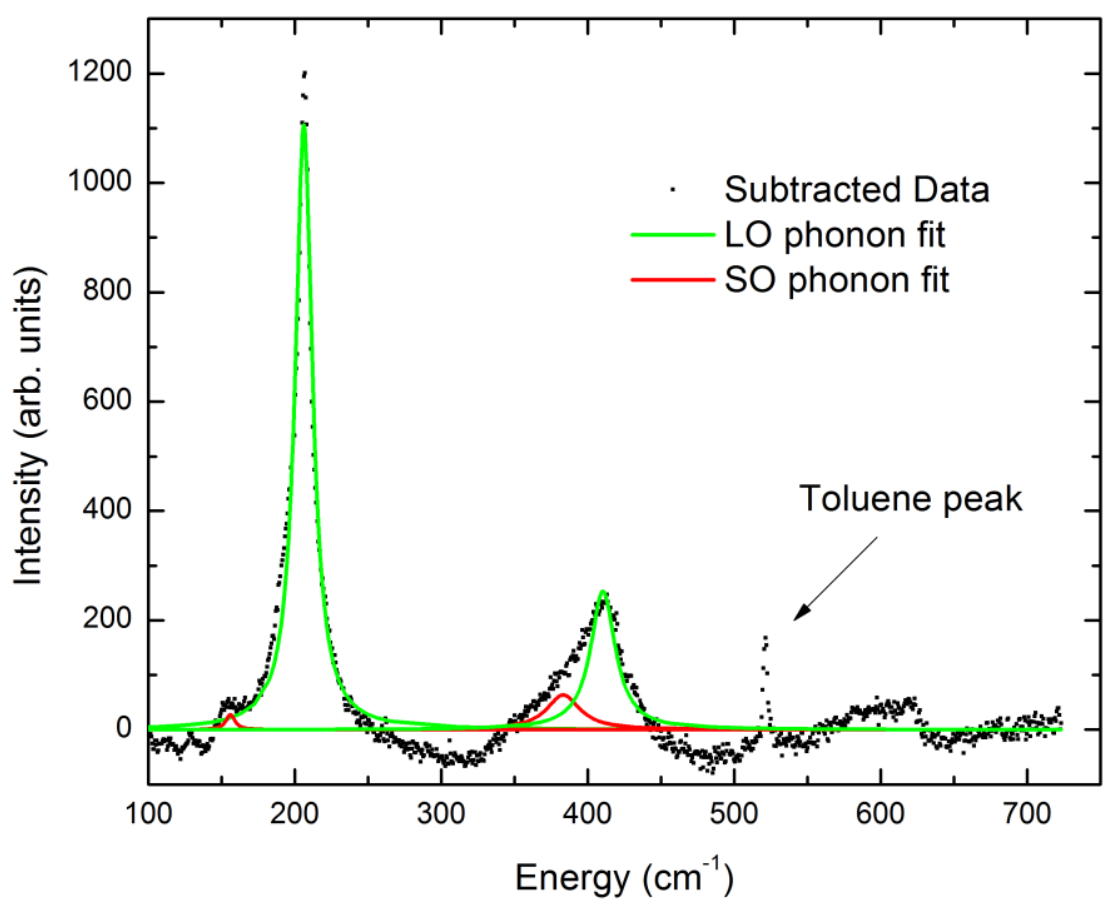

Figure 5.2. Resonance Raman spectrum of CdSe NCs, showing fundamental phonon peak at 206 $\mathrm{cm}^{-1}$ and overtone at $410 \mathrm{~cm}^{-1}$. A cumulative fit to the data results in two LO phonon peaks (green), and two SO phonon peaks at the leading edge of the LO phonon peaks (red).

In the wavepacket approach of Heller et al., Raman excitation profiles can be modeled as the Fourier transform of the dipole-dipole correlation function:

$$
\sigma_{i \rightarrow f}(\omega)=\left|\int_{-\infty}^{\infty}\langle f \mid i(t)\rangle e^{i \omega t-\frac{1}{2} \Theta^{2} t^{2}-\Gamma|t|}\right|^{2}
$$


Here $\langle f \mid i(t)\rangle$ is the correlation function, $\omega$ is the excitation frequency, $\Theta$ is an inhomogeneous broadening parameter, and $\Gamma$ is the homogeneous linewidth. For the Raman data, the state $\mid i(t)>$ in the equation is the ground state, $\mid 0(t)>$. In the case of harmonic potential energy surfaces:

$$
\begin{gathered}
\langle 0 \mid 0(t)\rangle=\prod_{k} e^{-S_{k} \cdot\left(1-e^{-i \omega_{k} t}\right)-i \omega_{e g} t} \\
\langle n \mid 0(t)\rangle=\prod_{k} S_{k}^{\frac{n}{2}} \cdot(-1)^{n} \cdot(n !)^{-\frac{1}{2}} \cdot\left(e^{-i \omega_{k} t}-1\right)^{n} \cdot\langle 0 \mid 0(t)\rangle
\end{gathered}
$$

Where $\mathrm{S}$ is the Huang-Rhys parameter, $\omega_{\mathrm{k}}$ is the phonon energy, $\omega_{\text {eg }}$ represents the energy difference between the ground and excited state potential energy surface minima, and $n$ is the order of the phonon (fundamental, first overtone, etc.).

Utilizing a value of $\Gamma=50 \mathrm{meV}$ for the homogeneous linewidth at room temperature ${ }^{15}$ and $\omega_{\mathrm{k}}=208 \mathrm{~cm}^{-1}$ for the optical phonon energy, the overtone:fundamental ratio of 0.331 can be reproduced with a value of $S=7.8$, suggesting that coupling to the surface state is three orders of magnitude greater than coupling to the core state and that values of coupling to the surface state on the order of $\mathrm{S} \sim 10$ should be expected. This result is qualitatively consistent with the coupling values obtained in the previous chapter, and differences between the two values can be ascribed to the possibility that not every NC in the ensemble measured using Raman spectroscopy has a surface-trapped exciton. The key point is that independent experiments have 
confirmed that electron-phonon coupling involving surface-trapped excitons is several orders of magnitude greater than for untrapped excitons, supporting a model of surface trapping that involves a substantial increase in coupling upon trapping.

\subsection{Hot carrier trapping}

In the previous chapter, the energy of excitation was set to ensure only the 1S state was populated and carrier trapping occurred exclusively from this state. Excitation with high energy photons can create excited or hot excitons. Hot excitons normally undergo a relaxation or cooling process to the $\mathrm{X} 1$ state. ${ }^{6,7,16,17}$. But these hot excitons may also experience direct trapping to surface states, which competes with the process of relaxation. If these trapped excitons cannot detrap back to the $1 \mathrm{~S}$ state prior to relaxation, then the quantum yield of the $1 \mathrm{~S}$ state would decrease as a result of this hot trapping. Thus changes in quantum yield could provide insight into the efficiency of the hot trapping process relative to the relaxation process. Several authors have suggested that there may be experimental evidence of a QY spectrum (excitation energy dependence to the QY), though experiments by different groups have often reached different conclusions about whether such a spectrum exists. ${ }^{3,15-18}$ Nevertheless, some groups have concluded that there is such a spectrum, and work from the Loomis groups has suggested a very strong state-dependence to the quantum yield (Figure 5.3)., ${ }^{6-21}$

Here, drawing from the same theoretical approach used to explain "cold" carrier trapping in Chapter 4, the effect of hot exciton trapping to the surface is considered. This treatment considers the conditions under which these nonequilibrium processes may manifest themselves in equilibrium CW measurements like PL and PLE. 


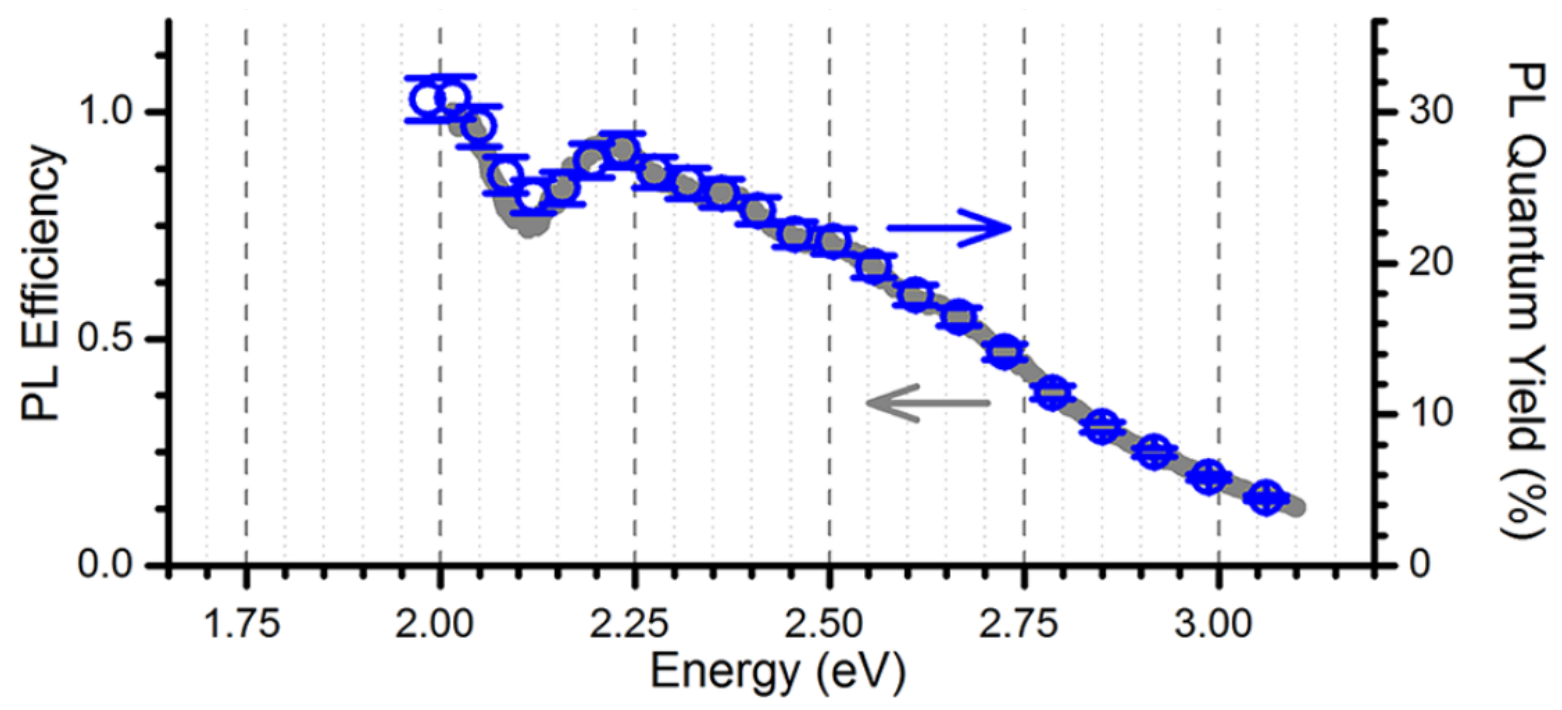

Figure 5.3. Excitation energy-dependence of PL quantum yield in CdSe nanocrystals.

Adapted with permission from "Excitation Energy Dependence of the Photoluminescence Quantum Yields of Core and Core/Shell Quantum Dots" Jessica Hoy, Paul J. Morrison, Lindsey K. Steinberg, William E. Buhro, and Richard A. Loomis. The Journal of Physical Chemistry Letters 20134 (12), 2053-2060. Copyright 2013 American Chemical Society.

Fig 5.4a shows a configuration coordinate diagram of NC states including several quantized exciton states. These states are denoted $\mathrm{X} 1\left(1 \mathrm{~S}_{\mathrm{e}}-1 \mathrm{~S}_{3 / 2}\right.$ in the effective mass approximation), $\mathrm{X} 2\left(1 \mathrm{~S}_{\mathrm{e}}-3 \mathrm{~S}_{3 / 2}\right)$ and $\mathrm{X} 3\left(1 \mathrm{P}_{\mathrm{e}}-1 \mathrm{P}_{3 / 2}\right){ }^{22}$ Also included is a surface state to which carrier trapping can occur. Here, exciton trapping takes place not only from the band edge (X1) state as in our previous works but also from higher excited states. The difference in trapping rate between states is dictated by the activation barrier to trapping 


$$
\Delta G^{\ddagger}=\frac{\left(\lambda+\Delta G^{0}\right)^{2}}{4 \lambda}
$$

Higher energy states will have different activation barriers to trapping and hence different trapping rates than the band edge (X1) feature.

In order to extend the cold electron transfer model to include hot exciton effects, the rates of hot exciton relaxation and hot exciton surface trapping must be known. Our prior works have produced measurements of both the cooling rate, $\mathrm{k}_{\mathrm{relax}}(\mathrm{E}),{ }^{6,7,16,17}$ as well as the surface trapping rate, $\mathrm{k}_{\text {trap }}(\mathrm{E})^{6,23-26}$, as a function of energy. These results indicate that the ratio of cooling (4.0 $\left.\mathrm{ps}^{-1}\right)$ to trapping $\left(0.65 \mathrm{ps}^{-1}\right)$ is $\sim 6$ for the $2 \mathrm{~S}$ state, while it grows to $\sim 10$ for higher states. ${ }^{23-26}$ Drawing from experimental values, these rates are used to perform a semi-empirical calculation, Fig. 5.4b. Here, the ratio of trapping to cooling rates was taken to linearly increase from 1:1 for the X2 state to 10:1 for the continuum. Within the framework of this approach, the quantum yield is now a function of both energy and temperature, QY $(\mathrm{E}, \mathrm{T})$ due to the possibility of thermally repopulating the $\mathrm{X} 1$ state. This semi-empirical approach predicts the existence of a QY spectrum. The extent of this effect is shown to be temperature-dependent; lower temperatures enable the effect to be seen more readily due to the inefficient detrapping from the surface state. 

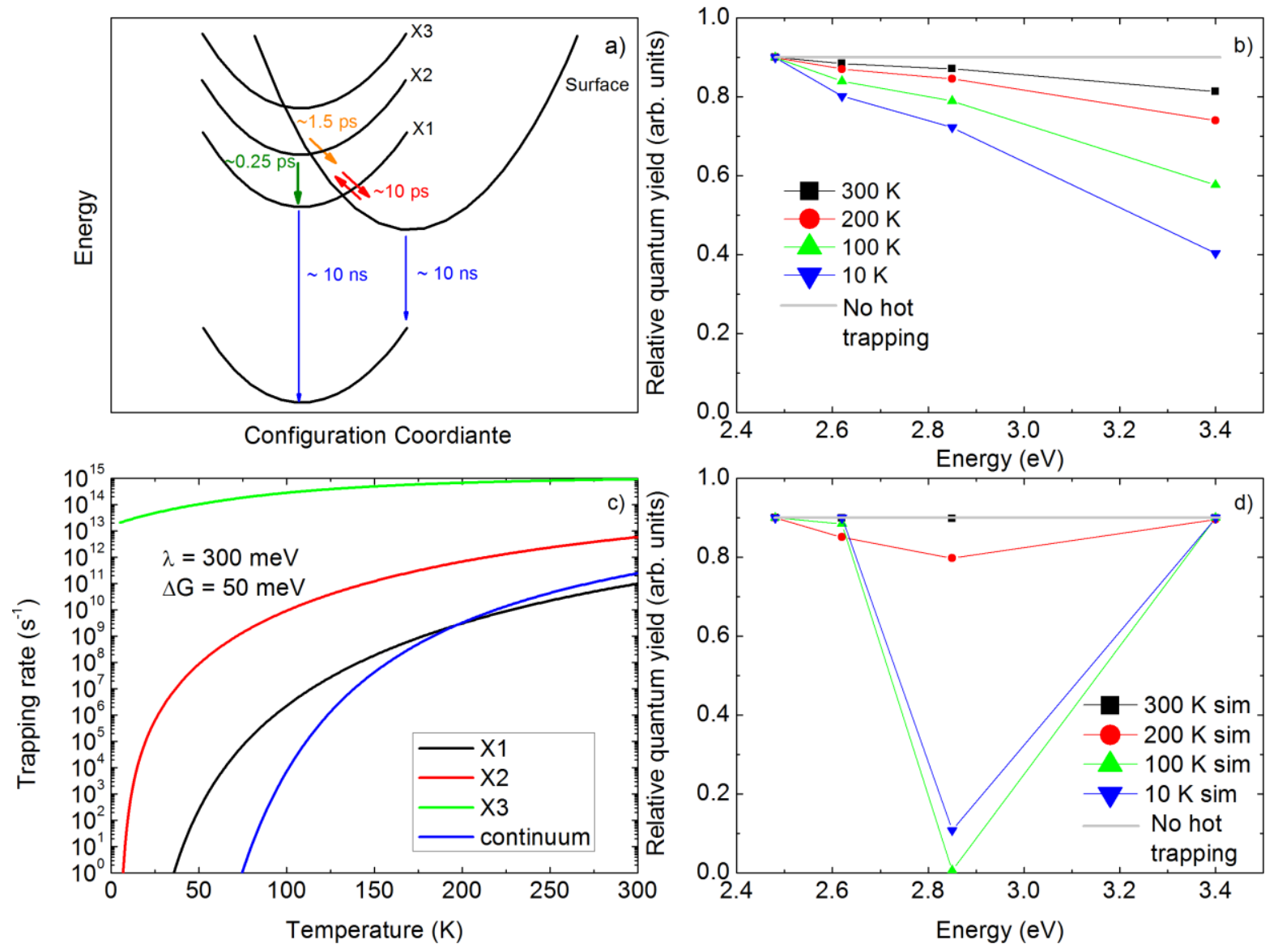

Figure 5.4. a) Schematic illustration of semiclassical electron transfer model of hot exciton surface trapping. b) Semi-empirical simulation of the total PL quantum yield spectrum at various temperatures. c) Calculated trapping rates using a microscopic model of surface charge trapping using semiclassical Marcus-Jortner electron transfer theory, assuming $\lambda=300 \mathrm{meV}$ and $\Delta \mathrm{G}$ (1Ssurface) $=50 \mathrm{meV}$. d) Microscopic simulation of total PL quantum yield spectrum at various temperatures. 
In addition to the semi-empirical approach, a fully microscopic model offers some insight. In the case of a fully microscopic model, the transition rates and trapping rates need to be explicitly obtained via the relevant state-to-state transition paths using equations 4.2 and 4.3. Here, the transition path from the core states to the surface states proceeds via semiclassical electron transfer. Hence one needs to compute the temperature-dependent ET rates from each initial excitonic state.

Fig 5.4c shows the results of this microscopic calculation, a simulation of the expected rates of hot carrier trapping for a given reorganization energy, $\lambda$, and X1 free energy difference, $\Delta \mathrm{G}$. The trapping rate increases with higher excitation energy (X2, X3) as the barrier to trapping is decreased, but decreases at very high energy as one enters the inverted region of electron transfer (continuum) where a very large negative value of $\Delta G^{0}$ actually leads to a large value of $\Delta G^{\ddagger}$ in Equation 5.6. Fig. 5.4d shows the result of the microscopic calculation of QY(E, T) using trapping rates from the microscopic model. The trends from the semi-empirical calculation are qualitatively reproduced here. Specifically, for some higher lying states at lower temperatures, carriers will be trapped at the surface and incapable of detrapping back to the band edge state, resulting in a lower quantum yield for the core state. But deviations arise at very high energy because the microscopic model predicts that a decrease in trapping rates far in the inverted region where activation energy increases. It is worth noting that this simple model does not consider any energy dependence to the overall electronic matrix elements. Higher states wavefunctions are more delocalized and thus more strongly coupled to the surface, increasing the rate with excitonic energy - as observed experimentally. Moreover, the density of states is greater at higher energy, resulting in a transfer from a higher to lower density of states which would also reduce electron transfer rates. Further development of this trapping model should 
account for the density of states and the greater delocalization of higher electronic states, a factor that has been accounted for previously by treating the system as a finite (rather than infinite) potential well. ${ }^{27}$ In addition, ultrafast studies that directly measure trapping rates without the possibility of detrapping could be conducted at several temperatures to provide further insight into the activation barriers involved in trapping. Nevertheless, this approach provides a framework for understanding the faster rates of trapping from higher excited states on a microscopic level.

In summary, the findings of the prior chapter with regard to the very high values of coupling for the surface state have been shown to be consistent with the results and conclusions of independent experiments. In addition, the surface trapping model of Chapter 4 may serve as the foundation for an understanding trapping from higher excitonic states, an issue that has generated great interest and controversy in recent years. 


\section{References}

1. $\quad$ Sagar, D. M.; Cooney, R. R.; Sewall, S. L.; Dias, E. A.; Barsan, M. M.; Butler, I. S.; Kambhampati, P., Size dependent, state-resolved studies of exciton-phonon couplings in strongly confined semiconductor quantum dots. Phys Rev B 2008, 77, 235321-14.

2. $\quad$ Sagar, D. M.; Cooney, R. R.; Sewall, S. L.; Kambhampati, P., State-Resolved ExcitonPhonon Couplings in CdSe Semiconductor Quantum Dots. J Phys Chem C 2008, 112, 91249127.

3. Bitto, H.; Huber, J. R., Molecular Quantum Beat Spectroscopy. Opt Commun 1990, 80, 184-198.

4. Sewall, S. L. Investigations of Charge Carrier Complexes in CdSe Nanocrystals Using State-Selective Ultrafast Spectroscopy McGill University, Montreal, 2010.

5. Bardeen, C. J.; Wang, Q.; Shank, C. V., Selective excitation of vibrational wave packet motion using chirped pulses. Phys Rev Lett 1995, 75, 3410-13.

6. Kambhampati, P., Hot Exciton Relaxation Dynamics in Semiconductor Quantum Dots: Radiationless Transitions on the Nanoscale. J Phys Chem C 2011, 115, 22089-22109.

7. Kambhampati, P., Unraveling the Structure and Dynamics of Excitons in Semiconductor Quantum Dots. Acc Chem Res 2011, 44, 1-13.

8. Krauss, T. D.; Wise, F. W., Coherent Acoustic Phonons in a Semiconductor Quantum Dot. Phys Rev Lett 1997, 79, 5102-5105.

9. Heller, E. J.; Sundberg, R. L.; Tannor, D., Simple Aspects of Raman-Scattering. Journal of Physical Chemistry 1982, 86, 1822-1833.

10. Shiang, J. J.; Risbud, S. H.; Alivisatos, A. P., Resonance Raman studies of the ground and lowest electronic excited state in cadmium sulfide nanocrystals. J Chem Phys 1993, 98, 8432-42.

11. Krauss, T. D.; Wise, F. W., Raman-scattering study of exciton-phonon coupling in PbS nanocrystals. Phys Rev B 1997, 55, 9860-9865.

12. Lange, H.; Machon, M.; Artemyev, M.; Woggon, U.; Thomsen, C., Effect of ZnS shell on the Raman spectra from CdSe nanorods. Physica Status Solidi-Rapid Research Letters 2007, 1, 274-276.

13. Lange, H.; Artemyev, M.; Woggon, U.; Niermann, T.; Thomsen, C., Experimental investigation of exciton-LO-phonon couplings in $\mathrm{CdSe} / \mathrm{ZnS}$ core/shell nanorods. Phys Rev $B$ 2008, 77, 193303.

14. Lange, H.; Mohr, M.; Artemyev, M.; Woggon, U.; Niermann, T.; Thomsen, C., Optical phonons in colloidal CdSe nanorods. Physica Status Solidi B-Basic Solid State Physics 2010, 247, 2488-2497.

15. Salvador, M. R.; Hines, M. A.; Scholes, G. D., Exciton-bath coupling and inhomogeneous broadening in the optical spectroscopy of semiconductor quantum dots. $J$ Chem Phys 2003, 118, 9380-9388.

16. Cooney, R. R.; Sewall, S. L.; Anderson, K. E. H.; Dias, E. A.; Kambhampati, P., Breaking the Phonon Bottleneck for Holes in Semiconductor Quantum Dots. Phys Rev Lett 2007, 98, 177403-4.

17. Cooney, R. R.; Sewall, S. L.; Dias, E. A.; Sagar, D. M.; Anderson, K. E. H.; Kambhampati, P., Unified picture of electron and hole relaxation pathways in semiconductor quantum dots. Phys Rev B 2007, 75, 245311. 
18. Rumbles, G.; Selmarten, D. C.; Ellingson, R. J.; Blackburn, J. L.; Yu, P. R.; Smith, B. B.; Micic, O. I.; Nozik, A. J., Anomalies in the linear absorption, transient absorption, photoluminescence and photoluminescence excitation spectroscopies of colloidal InP quantum dots. JOURNAL OF PHOTOCHEMISTRY AND PHOTOBIOLOGY A-CHEMISTRY 2001, 142, 187-195.

19. Tonti, D.; vanMourik, F.; Chergui, M., On the Excitation Wavelength Dependence of the Luminescence Yield of Colloidal CdSe Quantum Dots. Nano Lett. 2004, 4, 2483-2487.

20. Dias, E. A.; Grimes, A. F.; English, D. S.; Kambhampati, P., Single dot spectroscopy of two-color quantum dot/quantum shell nanostructures. J Phys Chem C 2008, 112, 14229-14232.

21. Hoy, J.; Morrison, P. J.; Steinberg, L. K.; Buhro, W. E.; Loomis, R. A., Excitation Energy Dependence of the Photoluminescence Quantum Yields of Core and Core/Shell Quantum Dots. J Phys Chem Lett 2013, 4, 2053-2060.

22. Efros, A. L.; Rosen, M., The electronic structure of semiconductor nanocrystals. Annual Review of Materials Science 2000, 30, 475-521.

23. Tyagi, P.; Kambhampati, P., False multiple exciton recombination and multiple exciton generation signals in semiconductor quantum dots arise from surface charge trapping. $J \mathrm{Chem}$ Phys 2011, 134, 094706-10.

24. Tyagi, P.; Cooney, R. R.; Sewall, S. L.; Sagar, D. M.; Saari, J. I.; Kambhampati, P., Controlling Piezoelectric Response in Semiconductor Quantum Dots via Impulsive Charge Localization. Nano Lett 2010, 10, 3062-3067.

25. Saari, J. I.; Dias, E. A.; Reifsnyder, D.; Krause, M. M.; Walsh, B. R.; Murray, C. B.; Kambhampati, P., Ultrafast Electron Trapping at the Surface of Semiconductor Nanocrystals: Excitonic and Biexcitonic Processes. J Phys Chem B 2013, 117, 4412-4421.

26. Sewall, S. L.; Cooney, R. R.; Anderson, K. E. H.; Dias, E. A.; Sagar, D. M.;

Kambhampati, P., State-resolved studies of biexcitons and surface trapping dynamics in semiconductor quantum dots. J Chem Phys 2008, 129, 084701.

27. Nosaka, Y., Finite Depth Spherical Well Model for Excited-States of Ultrasmall Semiconductor Particles - an Application. J Phys Chem-Us 1991, 95, 5054-5058. 


\section{Conclusions and future work}

\subsection{Conclusion}

This thesis demonstrated that a simple model involving Marcus-Jortner electron transfer transitions between the core state and a single surface state with strong coupling can account for the temperature-dependent behaviour of NCs. This surface state involves a trapped charge carrier which induces a strong polarization and strong electron-phonon coupling in the NC. This polarization, through a vibronic progression in emission, is responsible for the broad and strongly redshifted surface emission from NCs. This model suggests that manipulation of the surface chemistry of NCs holds promise for energy harvesting and other applications via exploitation of this surface state.

\subsection{Contributions to new discoveries}

The major advance presented in this thesis is a microscopic explanation of both the thermal response and the spectral properties of emission from core and surface states in NCs. Transfer of charge carriers in NCs is accounted for using Marcus-Jortner electron transfer theory. In this model, the two states are similar in energy, permitting thermal exchange, but different in coupling, accounting the different breadths and energies of their emission bands. Previous explanations employed phenomenological models to explain surface transfer and emission, but this work accounts for both properties on the basis of an established theory.

A second contribution of this work is to show that a single surface state rather than distribution of different states best explains the experimental data regarding surface emission. 
Thus, this work challenges the notion of the surface as a source of uncontrollable, energetically distinct defect states and instead presents it as the site of single state of defined energy and coupling, suggesting that the surface can be chemically controlled and exploited.

Finally, this work suggests that the variations in surface trapping rate with the energy of the initially excited quantum state may be explained in framework of Marcus electron transfer theory.

\subsection{Future directions}

Many studies have been conducted to vary the chemical composition of the NC surface to determine the effect on surface emission. ${ }^{1-3}$ Recently, a study was conducted of ultrasmall nanocrystals which analyzed the effect modifying the NC ligands on the parameters characterizing electron transfer (i.e. $\Delta \mathrm{G}$ and $\lambda$ ). ${ }^{4}$ In this study, variations in these parameters were correlated with the chemical properties of the ligands, analyzed through Marcus' treatment of outer-sphere reorganization energy. Additional work on a much wider variety of ligands is needed to draw conclusions regarding trends in chemical properties and electron-transfer transition parameters. Further work in this regard may yield additional insight and the development of a template for designing the chemical composition of the NC surface to achieve specific surface spectral properties.

While single-particle experiments have already demonstrated that individual NCs exhibit

surface emission over a broad range of wavelengths, ${ }^{5,6}$ these studies have been conducted at room temperature and with insufficient spectral resolution to resolve individual phonon lines, which have been observed for core emission. If the model of surface emission presented here is correct, a low-temperature single-particle study with sufficient energy resolution would provide 
the ideal evidence to confirm it. Such a study would directly show the vibrational structure of the surface state in the form of individually-resolved phonon lines within the surface emission band, confirming that its breadth is due to a Franck-Condon progression rather than a distribution of energy levels. 


\section{References}

1. Kuno, M.; Lee, J. K.; Dabbousi, B. O.; Mikulec, F. V.; Bawendi, M. G., The band edge luminescence of surface modified CdSe nanocrystallites: Probing the luminescing state. $J$ Chem Phys 1997, 106, 9869-9882.

2. Hasselbarth, A.; Eychmuller, A.; Weller, H., Detection of Shallow Electron Traps in

Quantum Sized Cds by Fluorescence Quenching Experiments. Chem Phys Lett 1993, 203, 271276.

3. Kalyuzhny, G.; Murray, R. W., Ligand effects on optical properties of CdSe nanocrystals. J Phys Chem B 2005, 109, 7012-7021.

4. Krause, M. M.; Mooney, J.; Kambhampati, P., Chemical and Thermodynamic Control of the Surface of Semiconductor Nanocrystals for Designer White Light Emitters. Acs Nano 2013, 7, 5922-5929.

5. $\quad$ Dukes, A. D.; Samson, P. C.; Keene, J. D.; Davis, L. M.; Wikswo, J. P.; Rosenthal, S. J., Single-Nanocrystal Spectroscopy of White-Light-Emitting CdSe Nanocrystals. J Phys Chem A 2011, 115, 4076-4081.

6. Layek, A.; De, S.; Thorat, R.; Chowdhury, A., Spectrally Resolved Photoluminescence Imaging of ZnO Nanocrystals at Single-Particle Levels. J Phys Chem Lett 2011, 2, 1241-1247. 


\section{Appendix A}

\section{A.1 Experimental details}

Solutions of colloidal CdSe and CdSe/ZnS NCs passivated with octadecylamine (ODA) ligands were used as received from NN Laboratories. The samples ranged in size from an average radius of $1.15 \mathrm{~nm}$ to $2.00 \mathrm{~nm}$. The size distribution of CdSe NCs was $5-10 \%$, while that of CdS NCs was $20 \%$. Solutions of colloidal CdS NCs passivated with oleic acid ligands were used as received from NN Laboratories. Cellulose triacetate was purchased from Sigma Aldrich. Polymer solutions of $5.5 \% \mathrm{wt} / \mathrm{wt}$ cellulose triacetate (CTA) in 1:9 methanol:dicholoromethane were prepared. Samples were prepared by thoroughly mixing NC solutions (original concentration: $0.5 \mathrm{~g} \mathrm{NC} / 100 \mathrm{~mL}$ toluene) in a 1:20 proportion with the CTA solutions. ${ }^{1,2}$ These samples were drop-cast in petri dishes which were covered and the solvent was allowed to evaporate over a period of at least 48 hours, producing films of NCs dispersed in hard polymer matrices.

Attempts were made to produce films in other polymer matrices. Notably, an effort was made to produce films in poly-methylmethacrylate, which has been employed previously in studying nanocrystals. However, these films were brittle and not at all robust. Since CTA had been successfully employed previously in the study of $\mathrm{NCs},{ }^{1,2}$ and since these films were durable and resulted in good quantum yield when illuminated, CTA films were used in the experiments.

Films were mounted in a Janis (STVP-100) flow cryostat, the vacuum chamber of which was evacuated continuously during the experiment using a turbomolecular pump. Sample 
temperatures were adjusted via $\mathrm{N}_{2}$ or LHe flow with a Lakeshore model 331 temperature controller. Steady-state absorption measurements were performed on a Varian Cary 5000 UV/Visible spectrophotometer. Photoluminescence spectra were measured on a Spex Fluromax2 spectrofluorometer. Slit widths of $1.8 \mathrm{~nm}$ for excitation and $2.8 \mathrm{~nm}$ for emission were employed for nearly all acquisitions and data was obtained in $1 \mathrm{~nm}$ intervals. Time-resolved data were obtained using a Picoquant Fluotime TCSPC setup employing an LDH 470 ps diode laser (Picoquant) with an excitation wavelength of $470 \mathrm{~nm}$. The instrument response function (IRF) was obtained on a LUDOX colloidal silica sample. The instrument resolution was approximately $350 \mathrm{ps.}$ Data was obtained over $100 \mathrm{~ns}$. Raman spectra were obtained using a Horiba Jobin Yvon LabRAM 800HR confocal Raman microscope using a $488 \mathrm{~nm}$ Ag ion laser.

For ultrafast spectroscopic measurements, data was acquired in the pump/probe configuration, the details of which have been previously described. ${ }^{3,4}$ The pump pulse was tuned by an Optical Parametric Amplifier (OPA) to be resonant with the band edge excitonic transition of the sample $(2.11 \mathrm{eV})$. The spectral full width at half maximum (FWHM) of the pump pulse was $56 \mathrm{meV}$. The unchirped pump pulse duration was $38 \mathrm{fs}$ in duration as measured by intensity autocorrelation. The probe pulse was tuned to the inflection point of the red rising edge of the electronic transition $(2.07 \mathrm{eV})$. Its spectral FWHM was $30 \mathrm{meV}$ and its duration was $59 \mathrm{fs}$ in duration as measured by intensity autocorrelation.

The optical density of the sample was $177 \mathrm{mOD}$. The pump pulse energy was maintained at $\sim 60 \mathrm{~nJ}$ throughout the experiment. Spot sizes were $286 \mu \mathrm{m}$ for the pump pulse and $78 \mu \mathrm{m}$ for the probe pulse. The average number of excitations $<\mathrm{N}>$ per particle was calculated to be 0.22 . 


\section{A.2 Comparison of results obtained in film vs solution}

To determine whether the use of polymer films had a determining influence on the results

obtained, a comparison was made between some basic data obtained on $\mathrm{NC}$ systems in film and in glassy-forming solvent. Ultimately the glassy-forming solvent (typically a mix of isopentane and methylcyclohexane) was not used to obtain data due to its unreliability at temperatures below $100 \mathrm{~K}$, at which it would sometimes crystallize. Still, the comparison shows that, broadly speaking, the same results are obtained in film and in solution. Values such as integrated peak area (Fig. A.1) and FWHM (Fig. A.2) do not change significantly for an identical sample in solution and in film.

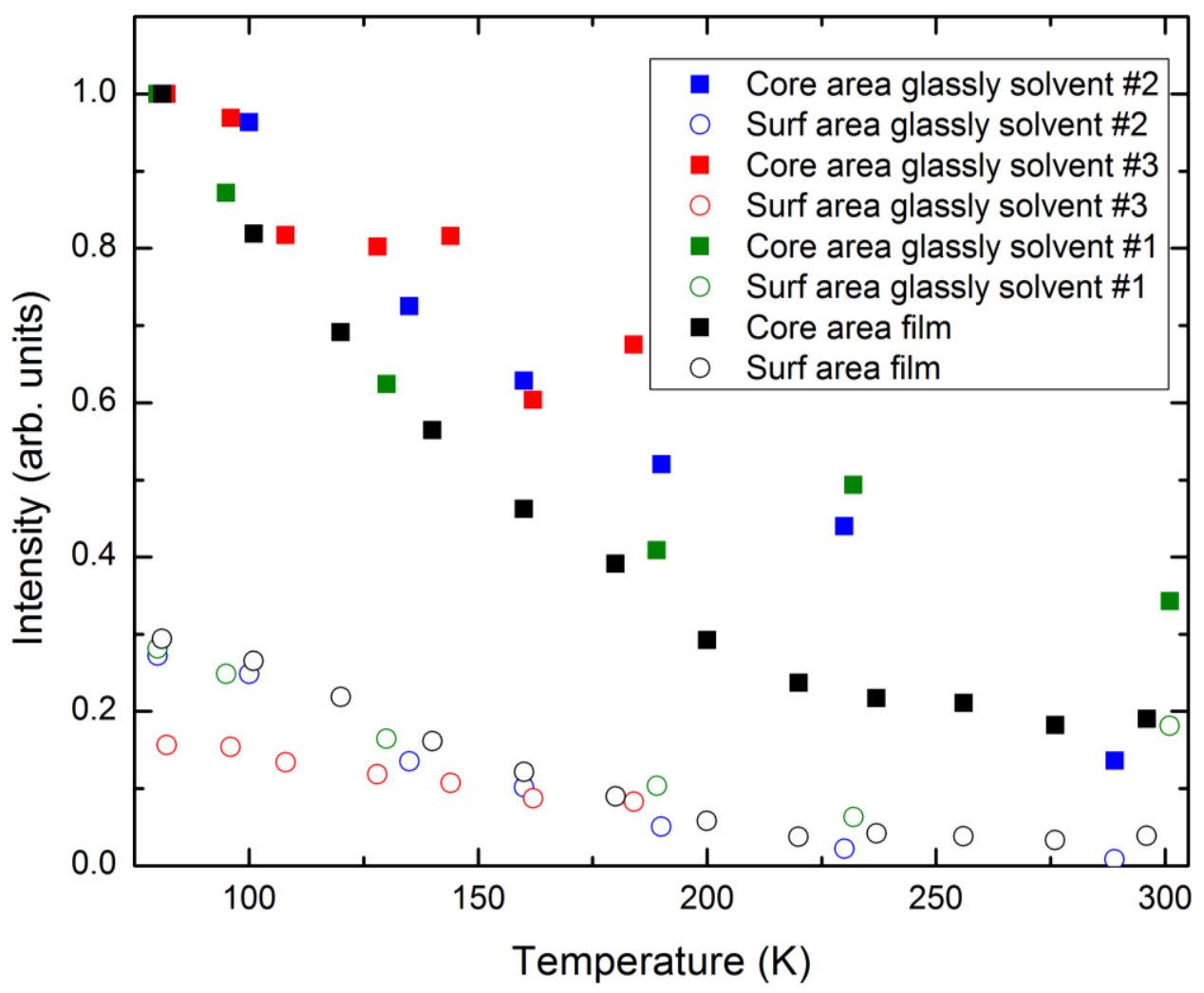

FIG. A.1. The core and surface peak areas of CdSe NCs $(\mathrm{R}=1.49 \mathrm{~nm})$ exhibit negligible 
difference when measured in solvent and in film. Peak areas are normalized to the core area at $80 \mathrm{~K}$.

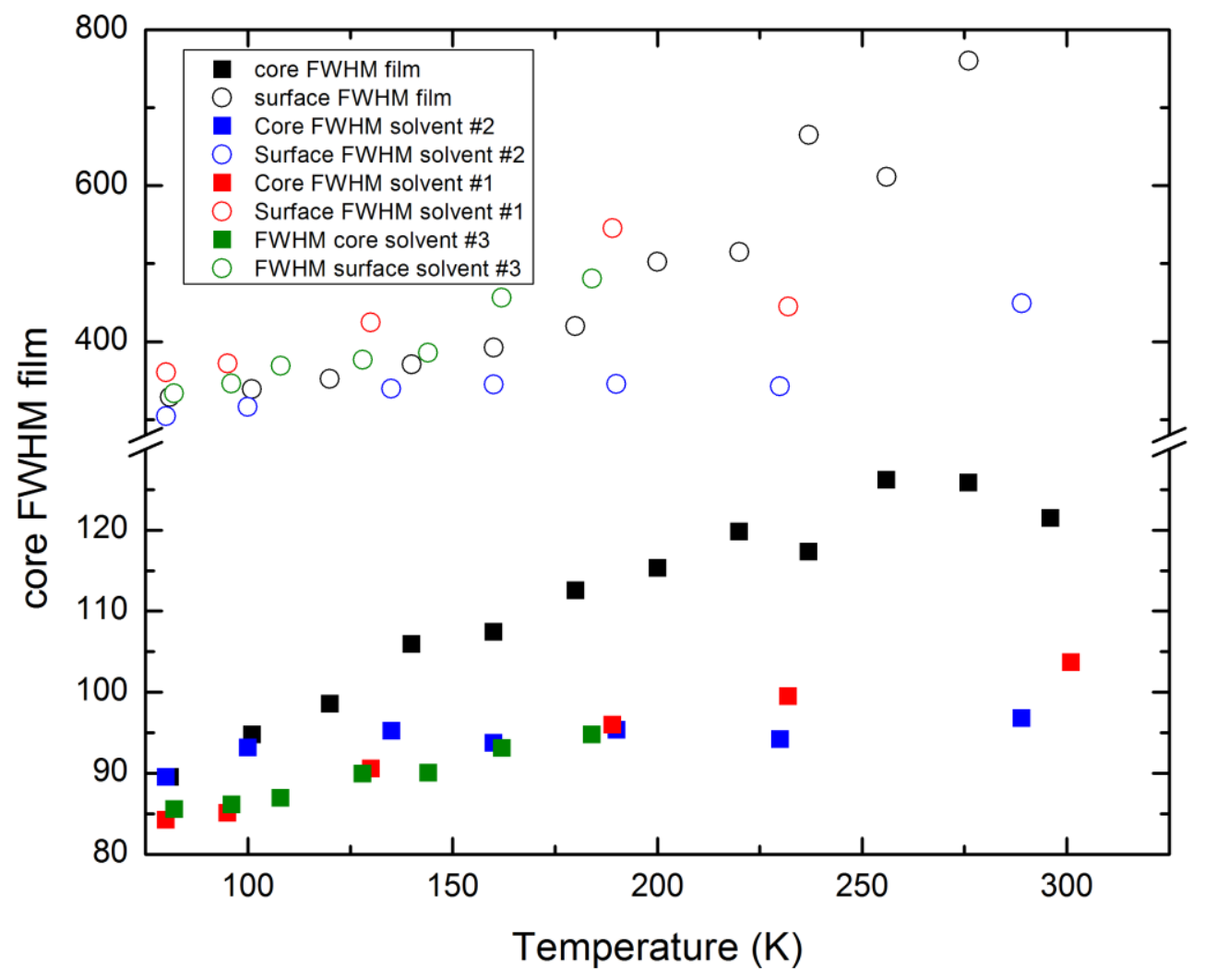

FIG. A.2. The core and surface peak FWHM values of CdSe NCs $(\mathrm{R}=1.49 \mathrm{~nm})$ exhibit small differences when measured in solvent and in film. The core FWHM is somewhat greater in film than in solvent. 


\section{References}

1. Abitbol, T.; Gray, D., CdSe/ZnS QDs embedded in cellulose triacetate films with hydrophilic surfaces. Chem Mater 2007, 19, 4270-4276.

2. Abitbol, T.; Gray, D. G., Incorporation into paper of cellulose triacetate films containing semiconductor nanoparticles. Cellulose 2009, 16, 319-326.

3. Sewall, S. L.; Cooney, R. R.; Anderson, K. E. H.; Dias, E. A.; Kambhampati, P., State-tostate exciton dynamics in semiconductor quantum dots. Phys Rev B 2006, 74.

4. $\quad$ Cooney, R. R.; Sewall, S. L.; Dias, E. A.; Sagar, D. M.; Anderson, K. E. H.;

Kambhampati, P., Unified picture of electron and hole relaxation pathways in semiconductor quantum dots. Phys Rev B 2007, 75. 


\section{Appendix B}

\section{B.1 Model equations}

Based on the mechanisms of population transfer described in Chapter 4, a series of coupled differential equations were constructed, all of which can be set to zero given the steadystate nature of the experiment:

$$
\begin{aligned}
& \frac{d n_{0}}{d t}=\frac{-n_{0}}{\tau_{0}}+\frac{n_{1}}{\tau_{r}}+\frac{n_{2}}{\tau_{r S}}=0 \\
& \frac{d n_{1}}{d t}=\frac{n_{0}}{\tau_{0}}-\frac{n_{1}}{\tau_{r}}-n_{1} \frac{2 \pi}{\hbar} H_{R P}{ }^{2}\left(\frac{\pi}{\hbar^{2} \lambda_{s} k_{b} T}\right)^{2} e^{-S} \sum_{n} \frac{S^{n}}{n !} e^{\frac{-\left(\Delta G^{0}+\lambda_{m}+n h<\omega>\right)^{2}}{4 \lambda_{m} k_{b} T}} \\
& +n_{2} \frac{2 \pi}{\hbar} H_{R P}^{2}\left(\frac{\pi}{\hbar^{2} \lambda_{S} k_{b} T}\right)^{\frac{1}{2}} e^{-S} \sum_{n} \frac{S^{n}}{n !} e^{\frac{-\left(\Delta G^{0}+\lambda_{m}+n h<\omega>\right)^{2}}{4 \lambda_{m} k_{b} T}} e^{\frac{\Delta G^{0}}{k_{b} T}}=0 \\
& \frac{d n_{2}}{d t}=n_{1} \frac{2 \pi}{\hbar} H_{R P}^{2}\left(\frac{\pi}{\hbar^{2} \lambda_{s} k_{b} T}\right)^{2} e^{-S} \sum_{n} \frac{S^{n}}{n !} e^{\frac{-\left(\Delta G^{0}+\lambda_{m}+n h<\omega>\right)^{2}}{4 \lambda_{m} k_{b} T}} \\
& -n_{2} \frac{2 \pi}{\hbar} H_{R P}^{2}\left(\frac{\pi}{\hbar^{2} \lambda_{s} k_{b} T}\right)^{\frac{1}{2}} e^{-S} \sum_{n} \frac{S^{n}}{n !} e^{\frac{-\left(\Delta G^{0}+\lambda_{m}+n h<\omega>\right)^{2}}{4 \lambda_{m} k_{b} T}} e^{\frac{\Delta G^{0}}{k_{b} T}} \\
& -\frac{n_{2}}{\tau_{r S}}=0
\end{aligned}
$$


The above equations were solved for $\mathrm{n}_{1}, \mathrm{n}_{2}$, and $\mathrm{n}_{3}$. These populations were easily related to total emission intensity, which were then scaled by the fraction of emitting NCs term:

$$
\begin{gathered}
A_{1 S}=\frac{n_{1}}{\tau_{r}} \\
A_{S}=\frac{n_{2}}{\tau_{r S}}
\end{gathered}
$$

\section{B.1. Treatment of the temperature-dependence of the quantum yield}

One aspect of the treatment of surface trapping presented in Chapter 4 that could be improved is how to account for the overall decrease quantum yield with temperature. In Chapter 4 , the temperature-dependence of the quantum yield was treated using the phenomenological equation 4.8 and 4.9 , in which the fraction of particles emitting from the core varied uniquely with temperature and the fraction emitting from the surface also varied uniquely with temperature. The need for temperature to affect the quantum yield via the emitting fraction rather than via a channel common to the entire ensemble arose from the temperatureindependence of the lifetime describe in equation 4.7.

As stated in Chapter 4, equations 4.8 and 4.9 are phenomenological. Here, to demonstrate that results of the surface-trapping model presented in Chapter 4 do not depend on this particular treatment of quantum yield, a slightly modified method of treating quantum yield is offered. The success of a different treatment of quantum yield shows that there are a number of valid approaches to this particular problem and the model of surface trapping presented in 
Chapter 4 is broadly valid and transcends any particular treatment. In "Challenge to the deeptrap model of the surface in semiconductor nanocrystals", ${ }^{1}$ it was shown that good fits to the data could be obtained even if a nonradiative channel common to all NCs was employed to explain the quantum yield temperature-dependence, even though such a mechanism is inconsistent with the experimental data on lifetimes. In this appendix, a different treatment of nonradiative processes with two modifications is offered. First, the fraction of emitting nanocrystals varies according to the same formula for both core and surface emission (Equation B.6). Second, the average number of surface states per NC is not fixed to $1: 1$ but rather set as a temperatureindependent constant. This constant then affects the final electronic density of states in the expression for the forward rate of reaction (4.2), modulating the electronic matrix element $\mathrm{H}_{\mathrm{RP}}$. The results of fitting with this model are presented in Figure B.1. Notably, both the fits and the output parameters from fits using either this method or the one presented in Chapter 4 are equivalent within statistical significance (Figure B.1 and Table B.1).

Fraction of emitting NCs (core and surface) $=1-A e^{\frac{-E a}{k_{b} T}}$ 

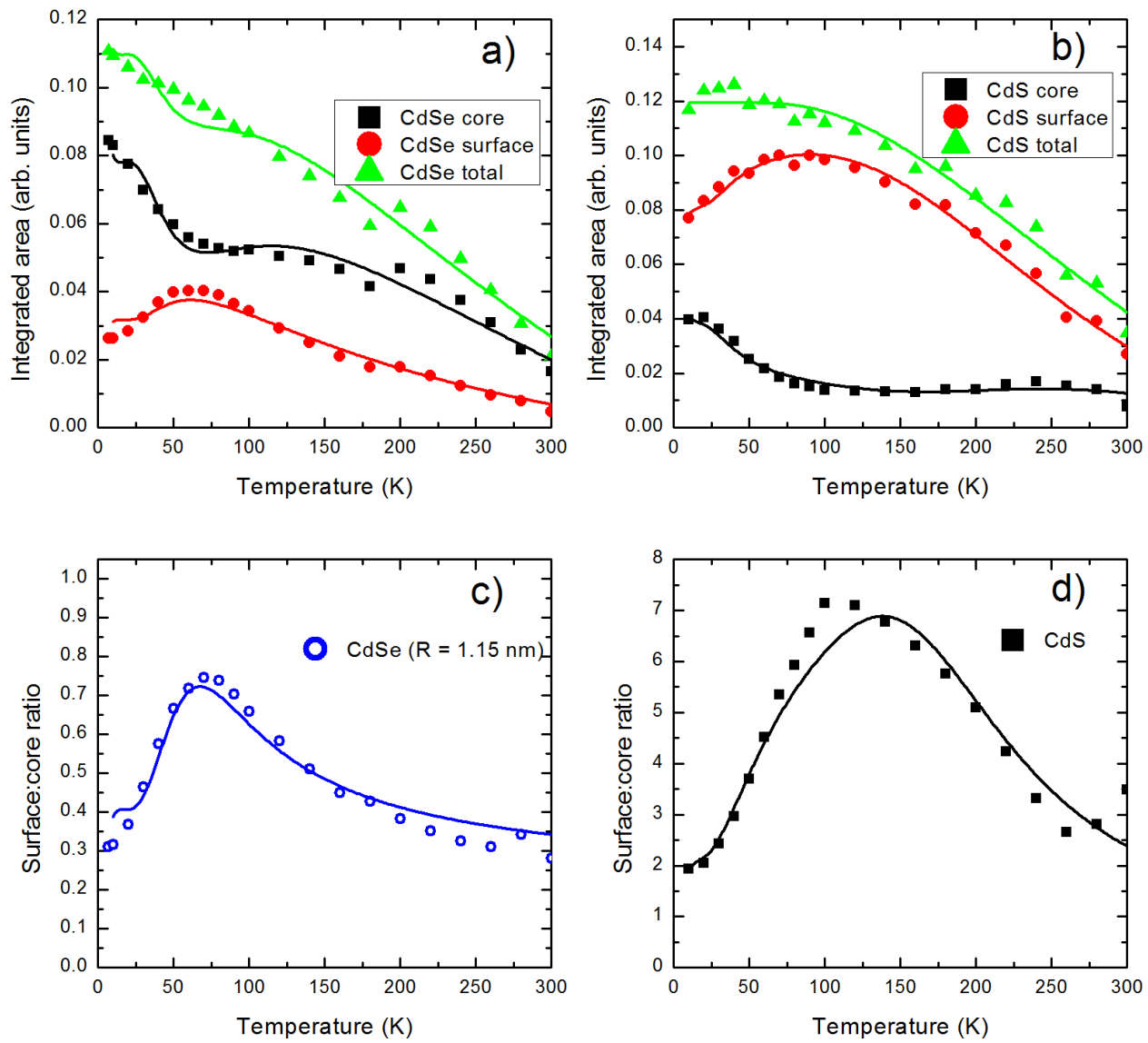

Figure B.1. Fits to $\mathrm{CdSe}$ and $\mathrm{CdS}$ data using an equation B.1 which treats the temperaturedependence of the core and surface quantum yield the same. The density of surface states is allowed to vary. 
Table B.1. Output parameters from fitting using several methods of accounting for the temperature-dependence of the quantum yield

\begin{tabular}{|l|l|l|l|l|}
\hline Sample & Treatment of QY & $\Delta \mathrm{G}(\mathrm{meV})$ & $\lambda(\mathrm{meV})$ & $\mathrm{S}$ \\
\hline \multirow{4}{*}{ CdSe 500 } & Equations 4.8, 4.9 & $13 \pm 1$ & $8 \pm 1$ & $23 \pm 2$ \\
\cline { 2 - 4 } & Equation B.6 & $13 \pm 1$ & $9 \pm 1$ & $23 \pm 2$ \\
\cline { 2 - 4 } & None; fit surface:core ratio as in section 4.4 & $18 \pm 2$ & $14 \pm 2$ & $22 \pm 3$ \\
\cline { 2 - 4 } & $\begin{array}{l}\text { Equations 4.8, 4.9; } \\
\text { Treat reverse rate using section B.2 }\end{array}$ & $10 \pm 1$ & $5 \pm 1$ & $23 \pm 2$ \\
\cline { 2 - 5 } & $\begin{array}{l}\text { Equation B.6; } \\
\text { Treat reverse rate using section B.2 }\end{array}$ & $21 \pm 3$ & $18 \pm 4$ & $22 \pm 6$ \\
\hline CdS & Equations 4.8, 4.9 & $66 \pm 7$ & $21 \pm 8$ & $21 \pm 18$ \\
\cline { 2 - 5 } & Equation B.6 & $67 \pm 8$ & $22 \pm 9$ & $21 \pm 19$ \\
\cline { 2 - 5 } & None; fit surface:core ratio as in section 4.4 & $62 \pm 7$ & $16 \pm 7$ & $21 \pm 16$ \\
\cline { 2 - 5 } & $\begin{array}{l}\text { Equations 4.8, 4.9; } \\
\text { Treat reverse rate using section B.2 }\end{array}$ & $60 \pm 70$ & $70 \pm 50$ & $20 \pm 70$ \\
\cline { 2 - 5 } & $\begin{array}{l}\text { Equation B.6; } \\
\text { Treat reverse rate using section B.2 }\end{array}$ & $60 \pm 10$ & $50 \pm 40$ & $20 \pm 30$ \\
\hline
\end{tabular}

\section{B.2. Treatment of reverse electron transfer rate}

In classical Marcus theory, reverse rates of reaction are calculated via detailed balance, in which $k_{\text {reverse }}=k_{\text {forward }} e^{-\frac{-\Delta G^{0}}{k_{b} T}}$ (Equation 4.3). ${ }^{2}$ In classical Marcus theory, this calculation is mathematically equivalent to substituting $-\Delta \mathrm{G}^{0}$ for $\Delta \mathrm{G}^{0}$ in Equation 3.28 to change the forward rate to the reverse rate. However, in Marcus-Jortner theory, these two treatments are not equivalent. Although the calculation of reverse rates according to Marcus-Jortner theory has not been treated in detailed, a recent theoretical paper by Dorner et al. ${ }^{3}$ employs detailed balance to calculate reverse rates. Private communication with Dorner and Vedral reveals that this treatment is arises due to the Crooks-Jarzynski relation ${ }^{4}$. In Chapter 4, detailed balance was used to calculate reverse rates. For comparison, the results of the calculation substituting $-\Delta \mathrm{G}^{0}$ for $\Delta \mathrm{G}^{0}$ is shown in Figure B.2 and table B.1. 

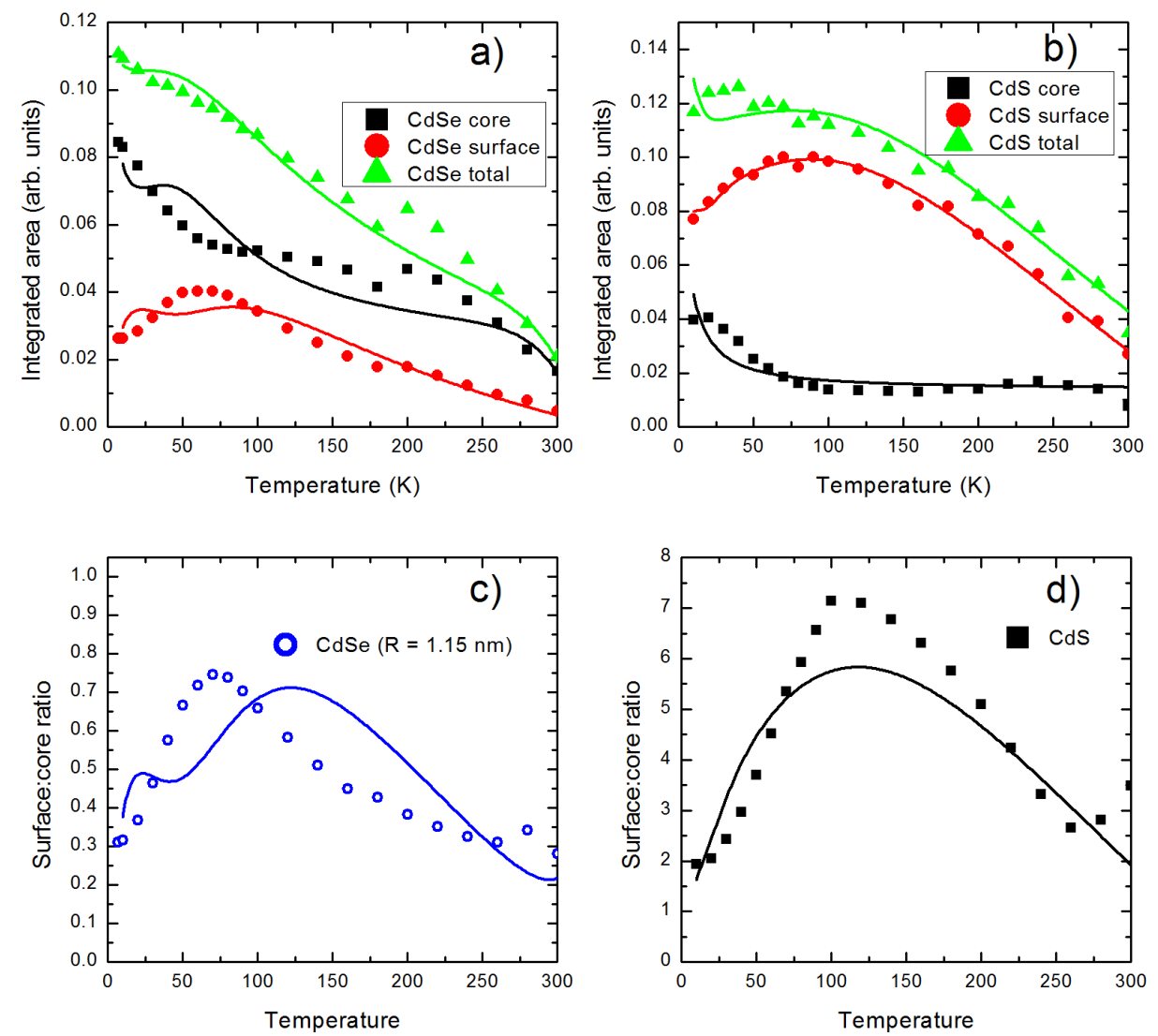

Figure B.2. Fits to $\mathrm{CdSe}$ and $\mathrm{CdS}$ data when substituting $-\Delta \mathrm{G}^{0}$ is substituted in the reverse reaction for $\Delta \mathrm{G}^{0}$ in the forward reaction. 


\section{References}

1. Mooney, J.; Krause, M. M.; Saari, J. I.; Kambhampati, P., Challenge to the deep-trap model of the surface in semiconductor nanocrystals. Phys Rev B 2013, 87.

2. Zusman, L. D., The Theory of Transitions between Electronic States - Application to Radiationless Transitions in Polar-Solvents. Chem Phys 1983, 80, 29-43.

3. Dorner, R.; Goold, J.; Heaney, L.; Farrow, T.; Vedral, V., Effects of quantum coherence in metalloprotein electron transfer. Phys Rev E 2012, 86.

4. R. Dorner and V. Vedral, private communication. 26 March 2013. 